\title{
Synthesis of Diazatricyclic Core of Madangamines from cis-Perhydroisoquinolines
}

Josefina Quirante*, Laura Paloma, Faïza Diaba, Xavier Vila, and Josep Bonjoch*

Laboratori de Química Orgànica, Facultat de Farmàcia, Institut de Biomedicina (IBUB), Universitat de Barcelona, Av. Joan XXIII s/n, 08028-Barcelona, Spain

Email: josep.bonjoch@ub.edu

\section{Supporting Information}

\section{Contents}

Copies of ${ }^{1} \mathrm{H}$ NMR and ${ }^{13} \mathrm{C}$ NMR spectra of all compounds, including COSY and HSQC spectra: 
General Methods, Physical Properties and Spectroscopic Measurements. All reactions were carried out under an argon atmosphere with dry, freshly distilled solvents under anhydrous conditions. Drying of organic extracts during workup of reactions was performed over anhydrous $\mathrm{Na}_{2} \mathrm{SO}_{4}$. Analytical TLC was performed on $\mathrm{SiO}_{2}$ (silica gel $60 \mathrm{~F}_{254}$, Merck) or $\mathrm{Al}_{2} \mathrm{O}_{3}$ (ALOX N/UV 254 , Polygram), and the spots were located with $1 \%$ aqueous $\mathrm{KMnO}_{4}$. Chromatography refers to flash chromatography and was carried out on $\mathrm{SiO}_{2}$ (silica gel 60, SDS, 230-240 mesh ASTM) or $\mathrm{Al}_{2} \mathrm{O}_{3}$ (aluminium oxide 90, Merck). Only noteworthy IR absorptions are listed $\left(\mathrm{cm}^{-1}\right)$. Chemical shifts of ${ }^{1} \mathrm{H}$ NMR spectra are reported in ppm downfield $(\delta)$ from $\mathrm{Me}_{4} \mathrm{Si}$. For ${ }^{13} \mathrm{C}$ NMR spectra chemical shifts are reported relative to the $\delta 77.00$ resonance of $\mathrm{CDCl}_{3}$. Unless otherwise noted ${ }^{1} \mathrm{H}$ and ${ }^{13} \mathrm{C}$ NMR spectra were recorded in $\mathrm{CDCl}_{3}$ solution at 400 and $100 \mathrm{MHz}$, respectively. Coupling constant are reported in $\mathrm{Hz}$. In ${ }^{13} \mathrm{C}$ NMR analysis always a DEPT experiment was included. All NMR assigments were made on the basis of twodimensional NMR experiments (gCOSY and gHSQC).

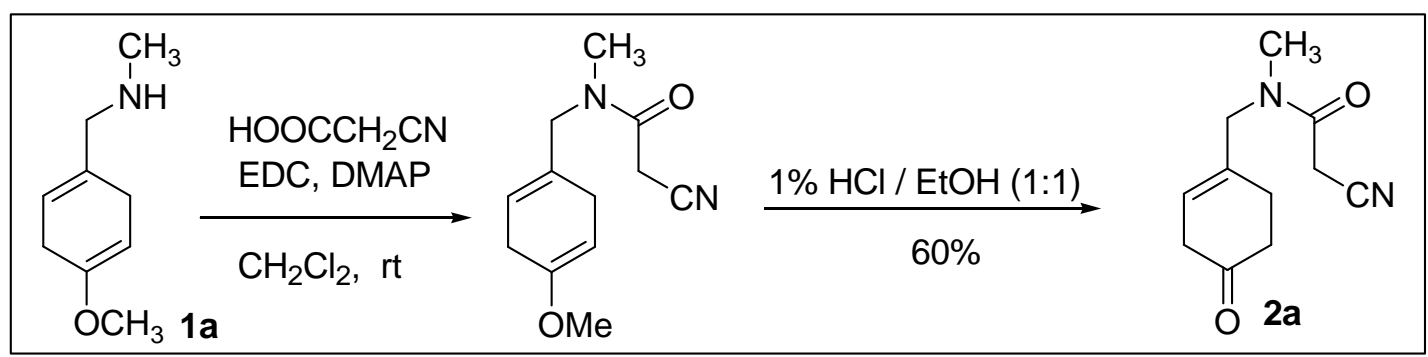

$\boldsymbol{N}$-Methyl-2-cyano- $\boldsymbol{N}$-(4-oxocyclohex-1-enylmethyl)acetamide (2a). To a cooled (0 $\left.{ }^{\circ} \mathrm{C}\right)$ solution of amine $\mathbf{1 a}^{\mathbf{1}}(2.03 \mathrm{~g}, 13.26 \mathrm{mmol})$ in $\mathrm{CH}_{2} \mathrm{Cl}_{2}(240 \mathrm{~mL})$ were added EDC (8.3 g, $15.9 \mathrm{mmol})$, cyanoacetic acid $(1.75 \mathrm{~g}, 19.9 \mathrm{mmol})$ and $\mathrm{Et}_{3} \mathrm{~N}(5.5 \mathrm{~mL}, 39.8$ mmol). The resulting solution was stirred overnight at rt, diluted with $\mathrm{CH}_{2} \mathrm{Cl}_{2}$ and washed sequentially with $5 \% \mathrm{NaHSO}_{4}$, saturated $\mathrm{NaHCO}_{3}$ and brine. The organic phase was concentrated to give the crude cyanoacetamide. To a solution of the above crude enol ether in $\mathrm{MeOH}(30 \mathrm{~mL})$ was added $1 \% \mathrm{HCl}(45 \mathrm{~mL})$. After the reaction mixture was stirred $5 \mathrm{~h}$ at $\mathrm{rt}, \mathrm{MeOH}$ was evaporated and the resulting aqueous phase was neutralized with solid $\mathrm{KOH}$ and extracted with $\mathrm{CH}_{2} \mathrm{Cl}_{2}$. The organic extracts were dried, concentrated, and the residue was purified by chromatography $\left(\mathrm{SiO}_{2}, \mathrm{CH}_{2} \mathrm{Cl}_{2} / \mathrm{MeOH}\right.$ 99:1) to give 2a (1.61 g, 60\% overall yield): IR( $\mathrm{NaCl}) 2928,2259,1711,1655,1487$; ${ }^{1} \mathrm{H}$ NMR (400 MHz, $\mathrm{CDCl}_{3}$, gCOSY) mixture of rotamers 2.25-2.45 (m, 2H, H-6), 2.45-2.60 (m, 2H, H-5), 2.92 and $2.95(2 \mathrm{~m}, 2 \mathrm{H}, \mathrm{H}-3), 2.99$ and $3.01\left(2 \mathrm{~s}, 3 \mathrm{H}, \mathrm{CH}_{3}\right), 3.52$ and $3.57\left(2 \mathrm{~s}, 2 \mathrm{H}, \mathrm{CH}_{2} \mathrm{CN}\right), 3.90$ and $4.05\left(2 \mathrm{~s}, 2 \mathrm{H}, \mathrm{NCH}_{2}\right), 5.45$ and $5.65(2 \mathrm{~m}, 1 \mathrm{H}, \mathrm{H}-2)$; ${ }^{13} \mathrm{C}$ NMR $(75 \mathrm{MHz}) 24.7$ and $25.2(\mathrm{C}-2), 26.2(\mathrm{C}-6), 34.7$ and $35.1\left(\mathrm{CH}_{3}\right), 37.7$ and $38.0(\mathrm{C}-5), 39.0$ and $39.3(\mathrm{C}-3), 52.7$ and $55.0\left(\mathrm{NCH}_{2}\right), 113.8(\mathrm{CN}), 120.7$ and $121.8(\mathrm{C}-$ 2), 132.4 and 133.1 (C-1), 161.9 (NCO), 209.2 (C-4); HRMS (ESI-TOF): calcd for $\mathrm{C}_{11} \mathrm{H}_{15} \mathrm{~N}_{2} \mathrm{O}_{2} 207.1128[\mathrm{M}+\mathrm{H}]^{+}$found 207.1123.

\footnotetext{
${ }^{1}$ Quirante, J.; Vila, X.; Paloma, L.; Guiu, J. M.; Bonjoch, J. Tetrahedron 2007, 63, 1372-1379.
} 


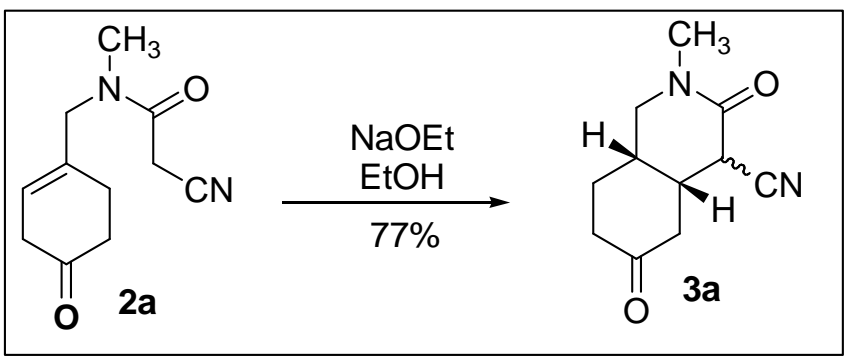

(4RS,4a $R S, 8 \mathrm{a} S R)$-and (4RS,4aSR,8a $R S)$-2-Methyl-3,6-dioxodecahydroisoquinoline4-carbonitrile (3a). To a solution of enone 2a (5.43 g, $26.4 \mathrm{mmol})$ in EtOH (190 mL) was added $\mathrm{NaOEt}(1 \mathrm{M}$ in EtOH, $13.2 \mathrm{~mL}, 13.2 \mathrm{mmol})$. After stirring the resulting solution at $\mathrm{rt}$ for $1 \mathrm{~h}, \mathrm{AcOH}(14 \mathrm{~mL})$ was added and the mixture was concentrated. The obtained residue was taken up in $\mathrm{CH}_{2} \mathrm{Cl}_{2}$ and washed with $5 \% \mathrm{NaHCO}_{3}$ and $\mathrm{H}_{2} \mathrm{O}$. The organic phase was dried and concentrated, and the resulting oil was purified by chromatography $\left(\mathrm{SiO}_{2}, \mathrm{CH}_{2} \mathrm{Cl}_{2} / \mathrm{MeOH} 95: 5\right)$ to afford 3a $(2.3 \mathrm{~g}, 77 \%)$ as a mixture of epimers in C-4 in a ratio 6:4 as an orange oil: IR $(\mathrm{NaCl}) 3491,2242,1713,1649 ;{ }^{1} \mathrm{H}$ NMR (400 MHz, $\mathrm{CDCl}_{3}, \mathrm{gCOSY}$ ) major epimer 1.90-2.10 (m, 2H, H-8), 2.45-2.50 (m, 1H, H-7), 2.53 (dd, $J=15.2,6 \mathrm{~Hz}, 1 \mathrm{H}, \mathrm{H}-5), 2.64$ (masked m, 1H, H-5), 2.65 (dm, $J=$ $12.2 \mathrm{~Hz}, 1 \mathrm{H}, \mathrm{H}-8 \mathrm{a}), 2.86$ (m, 1H, H-4a), 3.02 (s, 3H, CH $\mathrm{CH}_{3}, 3.29-3.35$ (m, 1H, H-4ax), $3.32(\mathrm{dd}, J=13.8,5.2 \mathrm{~Hz}, 1 \mathrm{H}, \mathrm{H}-1), 3.65$ (dd, $J=13,5.2 \mathrm{~Hz}, 1 \mathrm{H}, \mathrm{H}-1)$; minor epimer 2.00-2.10 (m, 2H, H-8), 2.37 (t, $J=13.2 \mathrm{~Hz}, 1 \mathrm{H}, \mathrm{H}-5 \mathrm{ax}), 2.40-2.45$ (m, 2H, H-7), 2.53 (masked m, 1H, H-8a), 2.72 (dq, $J=12,5 \mathrm{~Hz}, 1 \mathrm{H}, \mathrm{H}-4 \mathrm{a}), 2.77$ (m, 1H, H-5eq), 3.04 (s, $\left.3 \mathrm{H}, \mathrm{CH}_{3}\right), 3.41(\mathrm{dd}, J=12.8,6.4 \mathrm{~Hz}, 1 \mathrm{H}, \mathrm{H}-1), 3.64$ (t, $\left.J=13.2 \mathrm{~Hz}, 1 \mathrm{H}, \mathrm{H}-1\right), 3.87$ (d, $J$ $=5.2 \mathrm{~Hz}, 1 \mathrm{H}, \mathrm{H}-4 \mathrm{eq}) ;{ }^{13} \mathrm{C} \mathrm{NMR}\left(100 \mathrm{MHz}, \mathrm{CDCl}_{3}\right.$, gHSQC) major epimer 25.5 (C-8), 30.9 (C-8a), $35.5\left(\mathrm{CH}_{3}\right), 37.6$ (C-4), 38.4 (C-4a), 38.6 (C-7), 43.0 (C-5), 51.8 (C-1), $116.7(\mathrm{CN}), 160.2$ (C-3), $207.0(\mathrm{C}-6)$; minor epimer 26.0 (C-8), 30.2 (C-8a), 35.4 $\left(\mathrm{CH}_{3}\right), 36.0(\mathrm{C}-7), 36.6$ (C-4a), 39.4 (C-5), $39.7(\mathrm{C}-4), 49.0(\mathrm{C}-1), 115.2(\mathrm{CN}), 160.3$ (C-3), 206.7 (C-6); HRMS (ESI-TOF): calcd for $\mathrm{C}_{11} \mathrm{H}_{15} \mathrm{~N}_{2} \mathrm{O}_{2} 207.1128[\mathrm{M}+\mathrm{H}]^{+}$found 207.1122 .

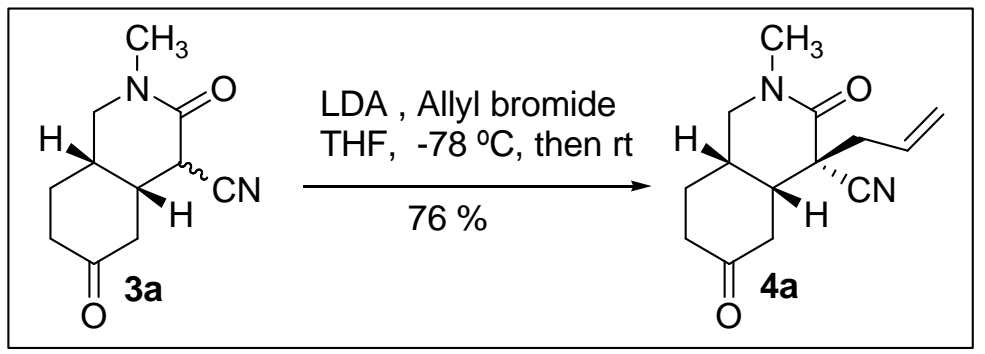

(4RS,4aSR,8a $R S$ )-4-Allyl-2-methyl-3,6-dioxodecahydroisoquinoline-4-carbonitrile

(4a). To a solution of $\mathbf{3 a}(4.17 \mathrm{~g}, 20.24 \mathrm{mmol})$ in THF $(210 \mathrm{~mL})$ cooled at $-78^{\circ} \mathrm{C}$ was added LDA (1.5 M in cyclohexane, $13.5 \mathrm{~mL}, 20.24 \mathrm{mmol})$ and the reaction mixture was stirred for $1 \mathrm{~h}$. Then, allyl bromide $(2.1 \mathrm{~mL}, 24.3 \mathrm{mmol})$ was added and stirring was continued for $2 \mathrm{~h}$ at the same temperature and overnight at $\mathrm{rt}$. After quenching with saturated $\mathrm{NH}_{4} \mathrm{Cl}(210 \mathrm{~mL})$ the resulting mixture was stirred, the organic phase was 
separated and the aqueous phase was extracted with $\mathrm{CH}_{2} \mathrm{Cl}_{2}$. The combined organic phases were dried, filtered and concentrated to give a residue, which was purified by chromatography $\left(\mathrm{SiO}_{2}, \mathrm{CH}_{2} \mathrm{Cl}_{2} / \mathrm{MeOH} 95: 5\right)$ to give $\mathbf{4 a}(3.8 \mathrm{~g}, 76 \%)$ as an orange oil: IR(NaCl) 3079, 2933, 2242, 1713, 1649, 1502 i 1449; ${ }^{1} \mathrm{H}$ NMR (400 MHz, $\mathrm{CDCl}_{3}$, gCOSY) $1.94(\mathrm{~m}, 1 \mathrm{H}, \mathrm{H}-8), 2.06(\mathrm{~m}, 1 \mathrm{H}, \mathrm{H}-8), 2.38$ (dtd, $J=12,5,1.2 \mathrm{~Hz}, 1 \mathrm{H}, \mathrm{H}-7 \mathrm{eq})$, 2.41 (m, 1H, H-5ax), 2.43-2.56 (m, 3H, H-4a and H-7), 2.61 (m, 1H, H.8a), 2.72 (dd, $J$ $\left.=14.2,8.6 \mathrm{~Hz}, 1 \mathrm{H}, \mathrm{CH}_{2}\right), 2.78(\mathrm{dm}, J=13.2 \mathrm{~Hz}, 1 \mathrm{H}, \mathrm{H}-5 \mathrm{eq}), 2.89$ (dd, $J=14,6 \mathrm{~Hz}$, $1 \mathrm{H}, \mathrm{CH}_{2}$ ), 3.04 (s, 3H, $\mathrm{CH}_{3}$ ), 3.39 (dd, $J=13.2,6 \mathrm{~Hz}, 1 \mathrm{H}, \mathrm{H}-1$ eq), 3.65 (dd, $J=12.8$, $11.2 \mathrm{~Hz}, 1 \mathrm{H}, \mathrm{H}-1 \mathrm{ax}), 5.25\left(\mathrm{dd}, J=17,1 \mathrm{~Hz}, 1 \mathrm{H},=\mathrm{CH}_{2}\right), 5.30\left(\mathrm{~d}, J=10 \mathrm{~Hz}, 1 \mathrm{H},=\mathrm{CH}_{2}\right)$, $5.87(\mathrm{~m}, 1 \mathrm{H},=\mathrm{CH}) ;{ }^{13} \mathrm{C} \mathrm{NMR}\left(100 \mathrm{MHz}, \mathrm{CDCl}_{3}, \mathrm{gHSQC}\right) 25.9(\mathrm{C}-8), 26.6(\mathrm{C}-8 \mathrm{a}), 35.7$ $\left(\mathrm{CH}_{3}\right), 36.2(\mathrm{C}-7), 38.6(\mathrm{C}-4 \mathrm{a}), 40.5(\mathrm{C}-5), 40.9\left(\mathrm{CH}_{2}\right), 49.1$ (C-4), $49.2(\mathrm{C}-1), 117.7$ $(\mathrm{CN}), 121.2\left(=\mathrm{CH}_{2}\right), 130.8(=\mathrm{CH}), 164.1(\mathrm{C}-3), 206.9$ (C-6); HRMS (ESI-TOF): calcd for $\mathrm{C}_{14} \mathrm{H}_{19} \mathrm{~N}_{2} \mathrm{O}_{2} 247.1441[\mathrm{M}+\mathrm{H}]^{+}$found 247.1439 .

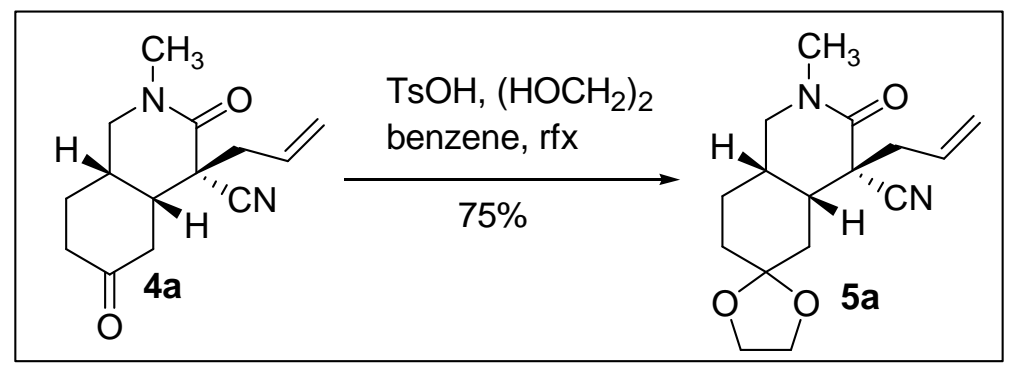

(4RS,4aSR,8aRS)-4-Allyl-2-methyl-3,6-dioxodecahydroisoquinoline-4-carbonitrile ethylene acetal (5a). To a solution of ketone $4 \mathbf{a}(3.34 \mathrm{~g}, 13.62 \mathrm{mmol})$ in benzene (290 $\mathrm{mL}$ ) were added TsOH (793 mg, $4.10 \mathrm{mmol})$ and ethyleneglycol $(0.9 \mathrm{~mL}, 16.34 \mathrm{mmol})$. After stirring the resulting solution at reflux for $3 \mathrm{~h}$ using a Dean-Stark apparatus, the reaction mixture was concentrated. The obtained residue was dissolved in EtOAc and washed with $1 \mathrm{M} \mathrm{NaOH}$. The organic extracts were dried, concentrated and purified by chromatography $\left(\mathrm{SiO}_{2}, \mathrm{CH}_{2} \mathrm{Cl}_{2} / \mathrm{MeOH} 98: 2\right)$ to yield acetal 5a $(2.98 \mathrm{~g}, 75 \%)$ as an orange oil: IR(NaCl) 2930, 2243, 1655, 1503, 1445; ${ }^{1} \mathrm{H}$ NMR (400 $\mathrm{MHz}, \mathrm{CDCl}_{3}$, gCOSY) 1.54 (t, $J=13.4 \mathrm{~Hz}, 1 \mathrm{H}, \mathrm{H}-5 \mathrm{ax}), 1.58-1.70$ (m, 2H, H-7ax and H-8eq), 1.73 $(\mathrm{dd}, J=13.2,4.4 \mathrm{~Hz}, 1 \mathrm{H}, \mathrm{H}-7 \mathrm{eq}), 1.81(\mathrm{~m}, 1 \mathrm{H}, \mathrm{H}-8 \mathrm{ax}), 2.10(\mathrm{dm}, J=13.2 \mathrm{~Hz}, 1 \mathrm{H}, \mathrm{H}-$ 5eq), 2.37 (dt, $J=13.6,3.6 \mathrm{~Hz}, 1 \mathrm{H}, \mathrm{H}-4 \mathrm{a}), 2.46$ (m, 1H, H-8a), 2.62 (dd, $J=14.2,8.6$ $\left.\mathrm{Hz}, 1 \mathrm{H}, \mathrm{CH}_{2}\right), 2.90$ (dd, $\left.J=14.2,6 \mathrm{~Hz}, 1 \mathrm{H}, \mathrm{CH}_{2}\right), 2.96\left(\mathrm{~s}, 3 \mathrm{H}, \mathrm{CH}_{3}\right), 3.15$ (dd, $J=12.6$, $6.8 \mathrm{~Hz}, 1 \mathrm{H}, \mathrm{H}-1 \mathrm{eq}), 3.45$ (dd, $J=12.8,12,1 \mathrm{H}, \mathrm{H}-1 \mathrm{ax}), 3.90-4.02\left(\mathrm{~m}, 4 \mathrm{H}, \mathrm{OCH}_{2}\right), 5.24$ $\left(\mathrm{dd}, J=17,1 \mathrm{~Hz}, 1 \mathrm{H},=\mathrm{CH}_{2}\right), 5.30\left(\mathrm{~d}, J=10.4 \mathrm{~Hz}, 1 \mathrm{H},=\mathrm{CH}_{2}\right), 5.93(\mathrm{~m}, 1 \mathrm{H},=\mathrm{CH}) ;{ }^{13} \mathrm{C}$ NMR (100 MHz, CDCl 3 , gHSQC) 24.8 (C-8), 25.3 (C-8a), 29.6 (C-7), 33.9 (C-5), 35.5 $\left(\mathrm{CH}_{3}\right), 37.3(\mathrm{C}-4 \mathrm{a}), 41.4\left(\mathrm{CH}_{2}\right), 48.7(\mathrm{C}-1), 50.3(\mathrm{C}-4), 64.49,64.52\left(\mathrm{OCH}_{2}\right), 107.8$ (C6), $118.3(\mathrm{CN}), 120.7\left(=\mathrm{CH}_{2}\right), 131.0(=\mathrm{CH}), 136.2$ (C-ipso), 164.9 (C-3); HRMS (ESI-TOF): calcd for $\mathrm{C}_{16} \mathrm{H}_{23} \mathrm{~N}_{2} \mathrm{O}_{3} 291.1703[\mathrm{M}+\mathrm{H}]^{+}$found 291.1696. 


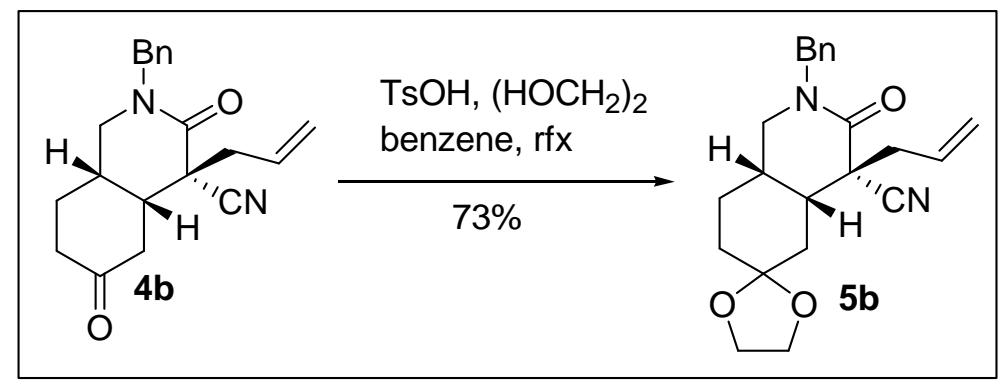

(4RS,4aSR,8aRS)-4-Allyl-2-benzyl-3,6-dioxodecahydroisoquinoline-4-carbonitrile ethylene acetal (5b). Following the above procedure to prepare 5a using ketone $\mathbf{4 b}$ (1.44 g, $4.47 \mathrm{mmol})$, the crude product was purified by chromatography $\left(\mathrm{SiO}_{2}\right.$, hexane/AcOEt 30:70) to give acetal $5 \mathbf{b}(1.25 \mathrm{~g}, 73 \%)$ as an orange oil: IR(NaCl) 2926, 1651, 1494, 1447, 1251; ${ }^{1} \mathrm{H}$ NMR (400 MHz, CDCl 3 , gCOSY) 1.10-1.80 (m, 5H), 1.75 (m, H-8ax), 2.11 (dm, $J=13.4$ Hz, 1H, H-5eq), 2.35 (m, 1H, H-4a), 2.38 (m, 1H, H-8a), 2.64 (dd, $J=14.2,8.6 \mathrm{~Hz}, 1 \mathrm{H}, \mathrm{CH}_{2}$ ), 2.95 (ddt, $J=14.6,1.4,1 \mathrm{~Hz}, 1 \mathrm{H}, \mathrm{CH}_{2}$ ), 3.06 (dd, $J=13,7.4 \mathrm{~Hz}, 1 \mathrm{H}, \mathrm{H}-1 \mathrm{eq}), 3.31$ (t, $J=12.2 \mathrm{~Hz}, 1 \mathrm{H}, \mathrm{H}-1 \mathrm{ax}), 3.88-4.01$ (m, 4H, $\mathrm{OCH}_{2}$ ), 4.32 and $4.83\left(2 \mathrm{~d}, J=14.4 \mathrm{~Hz}, 1 \mathrm{H}\right.$ each, $\left.\mathrm{CH}_{2} \mathrm{Ph}\right), 5.25(\mathrm{dd}, J=16.8,1.2 \mathrm{~Hz}, 1 \mathrm{H}$, $\left.=\mathrm{CH}_{2}\right), 5.31\left(\mathrm{~d}, J=10 \mathrm{~Hz}, 1 \mathrm{H},=\mathrm{CH}_{2}\right), 5.96(\mathrm{dddd}, J=16.8,10.4,8.6,6.2 \mathrm{~Hz}, 1 \mathrm{H}$, $=\mathrm{CH}), 7.20-7.35(\mathrm{~m}, 5 \mathrm{H}, \mathrm{ArH}) ;{ }^{13} \mathrm{C} \mathrm{NMR}\left(100 \mathrm{MHz}, \mathrm{CDCl}_{3}\right.$, gHSQC) $24.8(\mathrm{C}-8), 25.2$ (C-8a), 29.5 (C-7), 33.9 (C-5), 37.2 (C-4a), $41.6\left(\mathrm{CH}_{2}\right), 45.7$ (C-1), 50.5 (C-4), 50.9 $\left.\left(\mathrm{CH}_{2} \mathrm{Ph}\right), 64.5\left(\mathrm{OCH}_{2}\right), 107.8(\mathrm{C} 6), 118.3(\mathrm{CN}), 120.8=\mathrm{CH}_{2}\right), 127.9,128.2,128.9(\mathrm{Ar})$, $131.0(=\mathrm{CH}), 136.2$ (C-ipso), $165.0(\mathrm{C}-3)$; HRMS (ESI-TOF): calcd for $\mathrm{C}_{22} \mathrm{H}_{27} \mathrm{~N}_{2} \mathrm{O}_{3}$ 367.2016 [M+H] $]^{+}$found 367.2010.

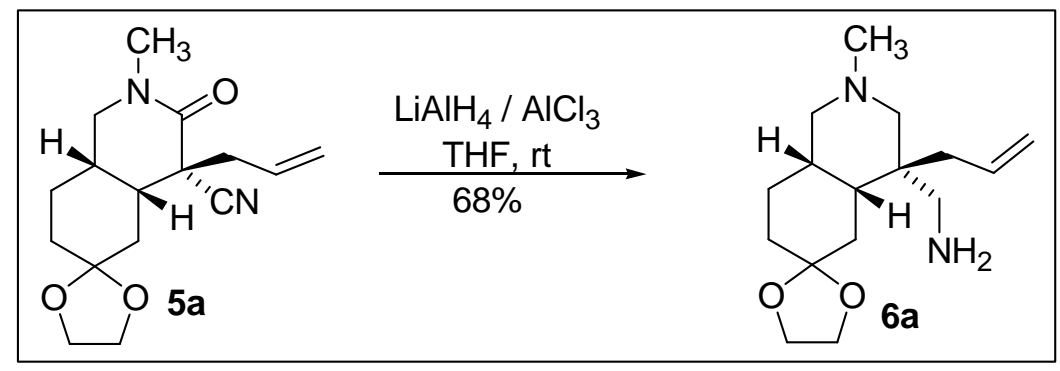

(4RS,4aSR,8a RS)-4-Allyl-4-aminomethyl-2-methyloctahydroisoquinolin-6(2H)-one ethylene acetal (6a). A solution of $\mathrm{LiAlH}_{4}(1 \mathrm{M}$ in THF, $5.2 \mathrm{~mL})$ and $\mathrm{AlCl}_{3}(230 \mathrm{mg}$, $1.72 \mathrm{mmol})$ in THF $(8 \mathrm{~mL})$ was stirred at $\mathrm{rt}$ for $15 \mathrm{~min}$. Then, nitrile $\mathbf{5 a}(0.5 \mathrm{~g}, 2.62$ mmol) in THF $(6 \mathrm{~mL})$ was added and stirring was continued for $5 \mathrm{~h}$. The reaction mixture was cooled at $0{ }^{\circ} \mathrm{C}$ and $30 \%$ aqueous $\mathrm{KOH}$ was added. The reaction mixture was extracted sequentially with $\mathrm{CH}_{2} \mathrm{Cl}_{2}, \mathrm{CHCl}_{3}$ and $\mathrm{CHCl}_{3} / i \mathrm{PrOH}(4: 1)$. The joined organic phases were dried, filtrered and concentrated, and purified by cromatography $\left(\mathrm{Al}_{2} \mathrm{O}_{3}, \mathrm{CH}_{2} \mathrm{Cl}_{2} / \mathrm{MeOH}\right.$ 95:5) to give 6a as a yellow oil $(0.33 \mathrm{~g}, 68 \%)$ : IR (NaCl) 3381, 3071, 2931, 1637, 1468; ${ }^{1} \mathrm{H}$ NMR (400 MHz, $\mathrm{CDCl}_{3}$, gCOSY) 1.45-1.62 (m), 1.67 (d, $J$ $=12 \mathrm{~Hz}, 1 \mathrm{H}, \mathrm{H}-3), 1.70-1.80(\mathrm{~m}), 1.83(\mathrm{~m}, 1 \mathrm{H}, \mathrm{H}-4 \mathrm{a}), 2.10-2.24(\mathrm{~m}, 2 \mathrm{H}, \mathrm{H}-1$ and $\mathrm{H}-$ 8a), 2.20-2.36 (m, 1H, $\left.\mathrm{CH}_{2}\right), 2.33$ (d, $\left.J=12 \mathrm{~Hz}, 1 \mathrm{H}, \mathrm{H}-3\right), 2.38-2.48$ (m, 3H, H-1 and 
$\left.\mathrm{CH}_{2} \mathrm{NH}_{2}\right), 2.61\left(\mathrm{dd}, J=14,6.8 \mathrm{~Hz}, 1 \mathrm{H}, \mathrm{CH}_{2}\right), 3.90-4.00\left(\mathrm{~m}, 4 \mathrm{H}, \mathrm{OCH}_{2}\right), 5.04-5.16(\mathrm{~m}$, $\left.2 \mathrm{H},=\mathrm{CH}_{2}\right), 5.84(\mathrm{~m}, 1 \mathrm{H},=\mathrm{CH}) ;{ }^{13} \mathrm{C} \mathrm{NMR}\left(100 \mathrm{MHz}, \mathrm{CDCl}_{3}\right.$, gHSQC) $26.8(\mathrm{C}-8), 29.3$ (C-7), 30.0 (C-5), 30.8 (C-8a), 36.2 (C-4a), $37.4\left(\mathrm{CH}_{2}\right), 40.3(\mathrm{C}-4), 44.7\left(\mathrm{NCH}_{2}\right), 46.7$ $\left(\mathrm{CH}_{3}\right), 55.4(\mathrm{C}-1), 57.6(\mathrm{C}-3), 64.1$ and $64.3\left(\mathrm{OCH}_{2}\right), 109.7(\mathrm{C}-6), 117.2\left(=\mathrm{CH}_{2}\right), 135.0$ $(=\mathrm{CH})$; HRMS (ESI-TOF): calcd for $\mathrm{C}_{16} \mathrm{H}_{29} \mathrm{~N}_{2} \mathrm{O}_{2} 281.2222[\mathrm{M}+\mathrm{H}]^{+}$found 281.2223.

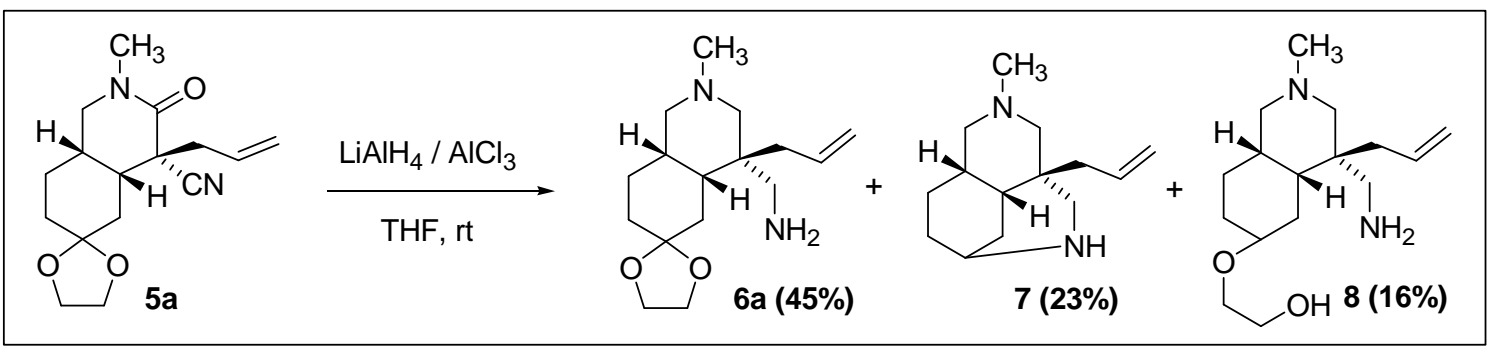

(4RS,4aSR,6SR,8aRS)-4-Allyl-3-methyl-6,4-(iminomethano)isoquinoline (7). A solution of $\mathrm{AlCl}_{3}(138 \mathrm{mg}, 1.04 \mathrm{mmol})$ in THF $(4 \mathrm{~mL})$ was added to $\mathrm{LiAlH}_{4}(1 \mathrm{M}$ solution in THF, $1.04 \mathrm{~mL})$. The mixture was stirred for $15 \mathrm{~min}$ and $5 \mathbf{a}(60 \mathrm{mg}, 0.21$ mmol) in THF $(1 \mathrm{~mL})$ was added dropwise. After stirring for $5 \mathrm{~h}$, the mixture was cooled to $0^{\circ} \mathrm{C}$ and quenched with aqueous $30 \%$ potassium hydroxide. The reaction mixture was extracted sequentially with $\mathrm{CH}_{2} \mathrm{Cl}_{2}, \mathrm{CHCl}_{3}$ and $\mathrm{CHCl}_{3} / i \operatorname{PrOH}(4: 1)$. The dried organic extracts were concentrated and purified by chromatography $\left(\mathrm{Al}_{2} \mathrm{O}_{3}\right.$, $\mathrm{CH}_{2} \mathrm{Cl}_{2}$ to $\mathrm{CH}_{2} \mathrm{Cl}_{2} / \mathrm{MeOH}$ 95:5) to yield in order of elution $6 \mathbf{a}(26 \mathrm{mg}, 45 \%$ ), tricyclic derivative 7 (11 $\mathrm{mg}, 23 \%)$ and aminoalcohol 8 (9 $\mathrm{mg}, 16 \%)$.

7: $\mathrm{IR}(\mathrm{NaCl}) 3357,2925,2856,1459,1377 ;{ }^{1} \mathrm{H} \mathrm{NMR}\left(400 \mathrm{MHz}, \mathrm{CDCl}_{3}, \mathrm{gCOSY}\right) 1.3$ (m, 1H, H-5), $1.42\left(\mathrm{~m}, W_{1 / 2}=11 \mathrm{~Hz}, 1 \mathrm{H}, \mathrm{H}-4 \mathrm{a}\right), 1.60(\mathrm{~m}, 1 \mathrm{H}, \mathrm{H}-8), 1.65-1.80(\mathrm{~m}, 2 \mathrm{H}$, H-7 and H-1), 1.85 (m, 1H, H-8a), 1.93 (dd, $J=10.8,3.2 \mathrm{~Hz}, 1 \mathrm{H}, \mathrm{H}-3), 2.05$ (dm, $J=$ $12.8 \mathrm{~Hz}, 1 \mathrm{H}, \mathrm{H}-5 \mathrm{eq}), 2.17$ (s, 3H, $\left.\mathrm{CH}_{3}\right), 2.10-2.30\left(\mathrm{~m}, 3 \mathrm{H}, \mathrm{H}-1\right.$ and $\left.\mathrm{CH}_{2}\right), 2.49$ (d, $J=$ $13.2 \mathrm{~Hz}, 1 \mathrm{H}, \mathrm{H}-10$ ), 2.55 (d, $J=11.2 \mathrm{~Hz}, 1 \mathrm{H}, \mathrm{H}-3$ ), 3.06 (brs, $W_{l / 2}=11 \mathrm{~Hz}, 1 \mathrm{H}, \mathrm{H}-6$ ), $3.57(\mathrm{~d}, J=13.6 \mathrm{~Hz}, 1 \mathrm{H}, \mathrm{H}-10), 5.05-5.15\left(\mathrm{~m}, 2 \mathrm{H},=\mathrm{CH}_{2}\right), 5.70-5.90(\mathrm{~m}, 1 \mathrm{H},=\mathrm{CH}) ;{ }^{13} \mathrm{C}$ NMR (100 MHz, CDCl 3 , gHSQC) 25.9 (C-4), 29.7 (C-11), 30.0 (C-5), 34.9 (C-12), 35.1 (C-3), 35.6 (C-9), $41.1\left(\mathrm{CH}_{2}\right), 45.3(\mathrm{C}-6), 46.8\left(\mathrm{CH}_{3}\right), 48.6(\mathrm{C}-8), 61.9(\mathrm{C}-10), 64.3$ (C-2), $117.9\left(=\mathrm{CH}_{2}\right), 134.1(=\mathrm{CH})$; HRMS (ESI-TOF): calcd for $\mathrm{C}_{14} \mathrm{H}_{25} \mathrm{~N}_{2} 221.2012$ $[\mathrm{M}+\mathrm{H}]^{+}$found 221.2008 .

8: IR(NaCl) 3365, 3072, 2928, 2791, 1638, 1447; ${ }^{1} \mathrm{H}$ NMR (400 $\mathrm{MHz}, \mathrm{CDCl}_{3}$, gCOSY) 1.00-2.20 (m), $2.21\left(\mathrm{~s}, 3 \mathrm{H}, \mathrm{OCH}_{3}\right), 2.20-2.80(\mathrm{~m}), 3.27(\mathrm{~m}, 1 \mathrm{H}, \mathrm{H}-6), 3.40-$ $4.00\left(\mathrm{~m}, 4 \mathrm{H}, \mathrm{OCH}_{2}\right), 5.00-5.20\left(\mathrm{~m}, 2 \mathrm{H},=\mathrm{CH}_{2}\right), 3.27(\mathrm{~m}, 1 \mathrm{H},=\mathrm{CH}) ;{ }^{13} \mathrm{C} \mathrm{NMR}(100$ $\mathrm{MHz}, \mathrm{CDCl}_{3}$, gHSQC) 27.3 (C-8), 27.7, 27.8 (C-5 and C-7), 29.7 (C-4a), 37.3 (C-8a), $37.5\left(\mathrm{CH}_{2}\right), 40.5(\mathrm{C}-4), 44.7\left(\mathrm{NCH}_{2}\right), 46.7\left(\mathrm{CH}_{3}\right), 55.8(\mathrm{C}-1), 57.9(\mathrm{C}-3), 62.1\left(\mathrm{OCH}_{2}\right)$, $69.0\left(\mathrm{CH}_{2} \mathrm{OH}\right), 79.4(\mathrm{C}-6), 117.2\left(=\mathrm{CH}_{2}\right), 135.0(=\mathrm{CH})$; HRMS (ESI-TOF): calcd for $\mathrm{C}_{16} \mathrm{H}_{29} \mathrm{~N}_{2} \mathrm{O}_{2} 283.2380[\mathrm{M}+\mathrm{H}]^{+}$found 283.2372. 


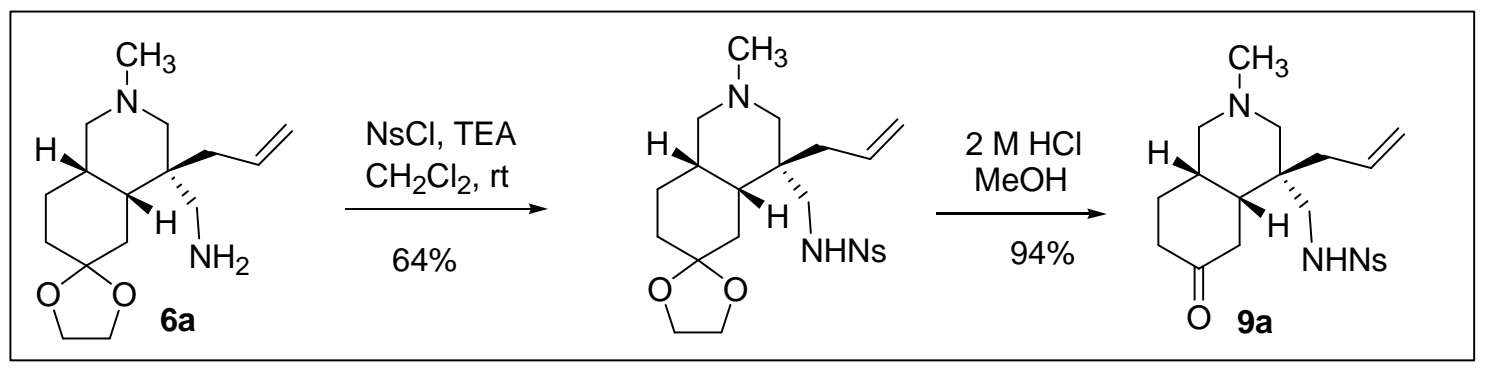

(4RS,4a $R S, 8 \mathrm{a} S R)-N$-(4-Allyl-2-methyl-6-oxodecahydroisoquinolin-4-ylmethyl)-2nitrobenzenesulfonamide (9a). To a cooled $\left(0^{\circ} \mathrm{C}\right)$ solution of $6 \mathbf{6}(327 \mathrm{mg}, 1.17 \mathrm{mmol})$ in $\mathrm{CH}_{2} \mathrm{Cl}_{2}(2.6 \mathrm{~mL})$ were added at $0{ }^{\circ} \mathrm{C}$ 2-nitrobenzenesulfonyl chloride $(285 \mathrm{mg}, 1.28$ mmol) and triethylamine $(0.17 \mathrm{~mL}, 1.17 \mathrm{mmol})$. The mixture was stirred at $\mathrm{rt}$ for $2 \mathrm{~h}$, quenched with $1 \mathrm{~N} \mathrm{HCl}(1 \mathrm{~mL})$, and extracted with $\mathrm{CH}_{2} \mathrm{Cl}_{2}, \mathrm{CHCl}_{3}$ and $\mathrm{CHCl}_{3} / i \operatorname{PrOH}$ (4:1). The organic extracts were dried, filtrered, concentrated, and purified by cromatography $\left(\mathrm{Al}_{2} \mathrm{O}_{3}, \mathrm{CH}_{2} \mathrm{Cl}_{2}\right)$ to give the nosyl derivative as a yellow foam (357 $\mathrm{mg}$, $64 \%$ ): IR(NaCl) 3350, 2934, 2793, 1540, 1351; ${ }^{1} \mathrm{H}$ NMR (400 MHz, $\mathrm{CDCl}_{3}, \mathrm{gCOSY}$ ) 1.55-1.61 (m, 6H, H-5, H-7, H-8), 1.72 (d, $J=11.6 \mathrm{~Hz}, 1 \mathrm{H}, \mathrm{H}-3), 1.88$ (m, 1H, H-4a), 2.13-2.22 (m, 5H, $\left.\mathrm{CH}_{3}, \mathrm{H}-8 \mathrm{a}, \mathrm{H}-1\right), 2.29$ (d, $\left.J=11.6 \mathrm{~Hz}, 1 \mathrm{H}, \mathrm{H}-3\right), 2.38$ (dd, $J=14.2,8$ $\mathrm{Hz}, 1 \mathrm{H}, \mathrm{CH}_{2}$ ), 2.44 (m, 1H, H-1), 2.60 (dd, $\left.J=14.2,7.2 \mathrm{~Hz}, 1 \mathrm{H}, \mathrm{CH}_{2}\right), 2.73$ and 2.77 $\left(2 \mathrm{~d}, J=12.4 \mathrm{~Hz}, 1 \mathrm{H}\right.$ each, $\left.\mathrm{CH}_{2} \mathrm{NHNs}\right), 3.91-4.02\left(\mathrm{~m}, 4 \mathrm{H}, \mathrm{OCH}_{2}\right), 5.06(\mathrm{~d}, J=10.8 \mathrm{~Hz}$, $\left.1 \mathrm{H},=\mathrm{CH}_{2}\right), 5.14\left(\mathrm{~d}, J=16.8 \mathrm{~Hz}, 1 \mathrm{H},=\mathrm{CH}_{2}\right), 5.77(\mathrm{~m}, 1 \mathrm{H},=\mathrm{CH}), 7.75,7.87$ and 8.09 (3m, 4H, NO $2 \mathrm{ArH}) ;{ }^{13} \mathrm{C} \mathrm{NMR} \mathrm{(100} \mathrm{MHz,} \mathrm{CDCl} 3$, gHSQC) 26.6 (C-8), 29.1 (C-8a), 30.0 (C-7), 30.1 (C-5), 36.1 (C-4a), $27.9\left(\mathrm{CH}_{2}\right), 39.6(\mathrm{C}-4 \mathrm{a}), 46.5\left(\mathrm{CH}_{3}\right), 46.7$ $\left(\mathrm{CH}_{2} \mathrm{NHNs}\right), 54.9(\mathrm{C}-1), 56.9(\mathrm{C}-3), 64.1$ and $64.2\left(\mathrm{OCH}_{2}\right), 109.2(\mathrm{C}-6), 118.7\left(=\mathrm{CH}_{2}\right)$, $125.4\left(\mathrm{NO}_{2} \mathrm{Ar}\right), 132.8(=\mathrm{CH}), 133.0$ (C-ipso), 133.7, $133.6\left(\mathrm{NO}_{2} \mathrm{Ar}\right), 148.1$ (C-ipso); HRMS (ESI-TOF): calcd for $\mathrm{C}_{22} \mathrm{H}_{31} \mathrm{~N}_{3} \mathrm{O}_{6} \mathrm{~S} 466.2006[\mathrm{M}+\mathrm{H}]^{+}$found 466.2007.

To a solution of the above acetal $(347 \mathrm{mg}, 0.75 \mathrm{mmol})$ in $\mathrm{MeOH}(15 \mathrm{~mL})$ was added 2 $\mathrm{M} \mathrm{HCl}(22 \mathrm{~mL})$ and the mixture was stirred overnight at $\mathrm{rt}$. The solvent was evaporated and the resulting mixture was basified with $\mathrm{K}_{2} \mathrm{CO}_{3}$ and extracted with $\mathrm{CH}_{2} \mathrm{Cl}_{2}$. The dried organic extracts were concentrated to give ketone 9a as a yellow oil (297 $\mathrm{mg}$, 94\%), which was used without further purification: IR $(\mathrm{NaCl}) 3344,2929,2793,1709$, 1350. ${ }^{1} \mathrm{H}$ NMR (400 MHz, $\mathrm{CDCl}_{3}$, gCOSY) 1.85 (dt, $\left.J=13.5,5 \mathrm{~Hz}, 1 \mathrm{H}, \mathrm{H}-8\right), 1.89-$ $1.97(\mathrm{~m}, 2 \mathrm{H}, \mathrm{H}-3$ and H-8), 2.01 (dt, $J=13.5,4.3 \mathrm{~Hz}, 1 \mathrm{H}, \mathrm{H}-4 \mathrm{a}), 2.12-2.20(\mathrm{~m}, \mathrm{H}$, $\left.\mathrm{CH}_{2}\right), 2.20-2.34$ (m, 3H, H-1 and H-7), $2.40\left(\mathrm{~s}, 3 \mathrm{H}, \mathrm{CH}_{3}\right), 2.47$ (d, $\left.J=12 \mathrm{~Hz}, 1 \mathrm{H}, \mathrm{H}-3\right)$, $2.53(\mathrm{~m}, 2 \mathrm{H}, \mathrm{H}-5), 2.64$ (dd, $J=10.4,2.6 \mathrm{~Hz}, 1 \mathrm{H}, \mathrm{H}-1), 2.77\left(\mathrm{~m}, 2 \mathrm{H}, \mathrm{NCH}_{2}\right) ;{ }^{13} \mathrm{C} \mathrm{NMR}$ (100 MHz, $\mathrm{CDCl}_{3}$, gHSQC) 28.7 (C-8), $29.5(\mathrm{C}-8 \mathrm{a}), 37.2(\mathrm{C}-7), 37.8\left(\mathrm{CH}_{2}\right), 38.0(\mathrm{C}-5)$, 39.4 (C-4), 40.0 (C-4a), $46.5\left(\mathrm{CH}_{3}\right), 46.8\left(\mathrm{NCH}_{2}\right), 55.1(\mathrm{C}-1), 57.1(\mathrm{C}-3), 119.2\left(=\mathrm{CH}_{2}\right)$, 125.6, 131.1, 132.9 (Ar), 133.0 (=CH), 133.7 (Ar), 148.1 (C-ipso), 210.9 (C-6); HRMS (ESI-TOF): calcd for $\mathrm{C}_{20} \mathrm{H}_{28} \mathrm{~N}_{3} \mathrm{O}_{5} \mathrm{~S} 422.1737[\mathrm{M}+\mathrm{H}]^{+}$found 422.1744 . 


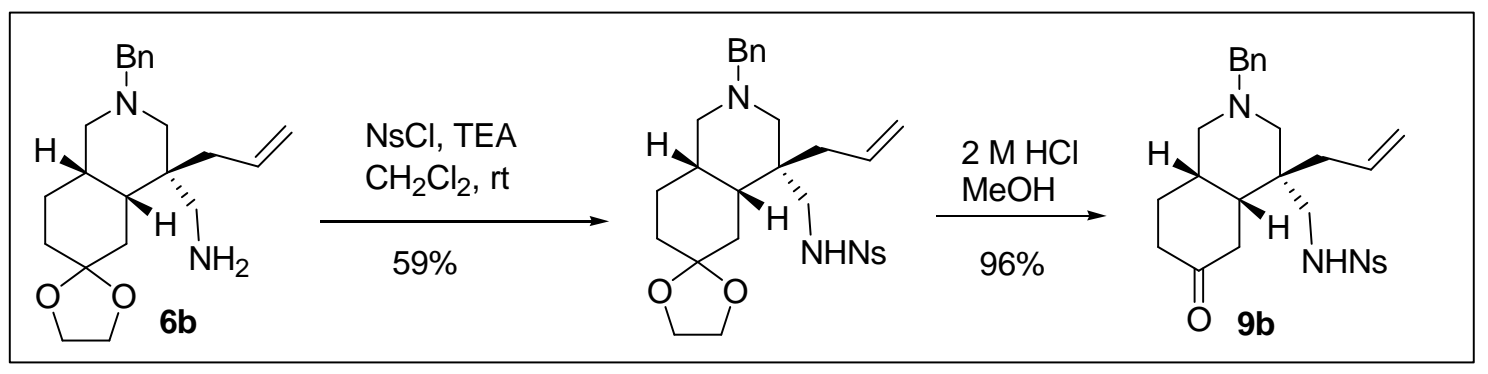

(4RS,4a $R S, 8 \mathrm{a} S R)-N$-(4-Allyl-2-benzyl-6-oxodecahydroisoquinolin-4-ylmethyl)-2-

nitrobenzenesulfonamide (9b). Starting from amine $\mathbf{6 b}(0.65 \mathrm{~g}, 1.76 \mathrm{mmol})$, operating as above, chromatography $\left(\mathrm{Al}_{2} \mathrm{O}_{3}\right.$, hexane $/ \mathrm{CH}_{2} \mathrm{Cl}_{2}$ 90:10) gave the corresponding nosyl derivative $(580 \mathrm{mg}, 59 \%)$ as a yellow foam: IR $(\mathrm{NaCl}) 3349,2933,2808,1540,1417$; ${ }^{1} \mathrm{H}$ NMR (400 MHz, CDCl 3 , gCOSY) 1.46-1.86 (m, 6H, H-5, H-7 and H-8), 1.73 (d, $J$ $=11.2 \mathrm{~Hz}, 1 \mathrm{H}, \mathrm{H}-3), 1.78(\mathrm{~m}, 1 \mathrm{H}, \mathrm{H}-8), 1.93(\mathrm{dm}, J=12 \mathrm{~Hz}, 1 \mathrm{H}, \mathrm{H}-4 \mathrm{a}), 2.18-2.31(\mathrm{~m}$, $2 \mathrm{H}, \mathrm{H}-1$ and $\mathrm{H}-3), 2.22-2.27\left(\mathrm{~m}, 3 \mathrm{H}, \mathrm{CH}_{2}, \mathrm{H}-3, \mathrm{H}-8 \mathrm{a}\right), 2.35(\mathrm{dd}, J=14.2,7.6 \mathrm{~Hz}, 1 \mathrm{H}$ $\mathrm{CH}_{2}$ ), 2.48 (dd, $\left.J=19,13 \mathrm{~Hz}, 1 \mathrm{H}, \mathrm{H}-1\right), 2.63\left(\mathrm{dd}, J=12.2,5.8 \mathrm{~Hz}, 1 \mathrm{H}, \mathrm{NCH}_{2}\right), 2.70-$ $2.80\left(\mathrm{~m}, 2 \mathrm{H}, \mathrm{CH}_{2}\right.$ and $\left.\mathrm{NCH}_{2}\right), 3.33$ and $3.48\left(2 \mathrm{~d}, J=12.8 \mathrm{~Hz}, 1 \mathrm{H}\right.$ each, $\left.\mathrm{CH}_{2} \mathrm{Ph}\right), 3.88-$ $3.98\left(\mathrm{~m}, 4 \mathrm{H}, \mathrm{OCH}_{2}\right), 4.97$ (dd, $\left.J=10.4,2.4 \mathrm{~Hz}, 1 \mathrm{H},=\mathrm{CH}_{2}\right), 5.08(\mathrm{~d}, J=17.2 \mathrm{~Hz}, 1 \mathrm{H}$, $\left.=\mathrm{CH}_{2}\right), 5.63(\mathrm{~m}, 1 \mathrm{H},=\mathrm{CH}), 7.10-7.28(\mathrm{~m}, 5 \mathrm{H}, \mathrm{ArH}), 7.72,7.83,8.05(3 \mathrm{~m}, 4 \mathrm{H}$, $\left.\mathrm{NO}_{2} \mathrm{ArH}\right) ;{ }^{13} \mathrm{C} \mathrm{NMR}\left(100 \mathrm{MHz}, \mathrm{CDCl}_{3}\right.$, gHSQC) 26.7 (C-8), 29.2 (C-8a), 29.9 (C-7), 30.3 (C-5), 36.9 (C-4a), $38.2\left(\mathrm{CH}_{2}\right), 39.8(\mathrm{C}-4), 46.7\left(\mathrm{NCH}_{2}\right), 53.0(\mathrm{C}-1), 54.3$ (C-3), $63.1\left(\mathrm{CH}_{2} \mathrm{Ph}\right), 64.1$ and $64.3\left(\mathrm{OCH}_{2}\right), 109.3(\mathrm{C}-6), 118.6\left(=\mathrm{CH}_{2}\right), 125.4,127.0,128.2$, 128.7, 131.3, 132.7, 133.1, 133.5, 134.0, 138.7(=CH and Ar), 148.1 (C-ipso); HRMS (ESI-TOF): calcd for $\mathrm{C}_{28} \mathrm{H}_{36} \mathrm{~N}_{3} \mathrm{O}_{6} \mathrm{~S} 542.2309[\mathrm{M}+\mathrm{H}]^{+}$found 542.2316.

Starting from the above acetal $(0.58 \mathrm{~g}, 1.04 \mathrm{mmol})$ and operating as above, ketone $9 \mathrm{~b}(0.51 \mathrm{~g}, 96 \%)$ was isolated as a yellow oil, which was submitted to the next reaction without further purification: $\mathrm{IR}(\mathrm{NaCl}) 3346,2924,1709,1540,1357 ;{ }^{1} \mathrm{H}$ NMR (400 MHz, $\mathrm{CDCl}_{3}, \mathrm{gCOSY}$ ) 1.78-1.92 (m, 2H, H-8), 1.95 (d, J=12 Hz, 1H, H-3), 2.05 $(\mathrm{dm}, J=13.2 \mathrm{~Hz}, 1 \mathrm{H}, \mathrm{H}-4 \mathrm{a}), 2.18-2.27$ (m, 3H, H-5 and H-7), 2.37-2.43(m, 3H, H-1, H5 and $\mathrm{H}-8 \mathrm{a}), 2.45(\mathrm{~d}, J=12 \mathrm{~Hz}, 1 \mathrm{H}, \mathrm{H}-3), 2.47\left(\mathrm{dd}, J=15.2,8.4 \mathrm{~Hz}, 1 \mathrm{H}, \mathrm{CH}_{2}\right), 2.62-$ $2.78\left(\mathrm{~m}, 4 \mathrm{H}, \mathrm{H}-1, \mathrm{CH}_{2}, \mathrm{NCH}_{2}\right), 3.46$ and $3.53\left(2 \mathrm{~d}, J=13.4 \mathrm{~Hz}, 2 \mathrm{H}, \mathrm{CH}_{2} \mathrm{Ph}\right), 5.02(\mathrm{dd}, J$ $\left.=10,2 \mathrm{~Hz}, 1 \mathrm{H},=\mathrm{CH}_{2}\right), 5.13\left(\mathrm{dd}, J=17,1.4 \mathrm{~Hz}, 1 \mathrm{H},=\mathrm{CH}_{2}\right), 5.38(\mathrm{t}, J=6.4 \mathrm{~Hz}, 1 \mathrm{H}$, $\mathrm{NH}), 5.62(\mathrm{~m}, 1 \mathrm{H},=\mathrm{CH}), 7.20-7.40(\mathrm{~m}, 5 \mathrm{H}, \mathrm{ArH}), 7.73,7.85,8.06\left(3 \mathrm{~m}, 4 \mathrm{H}, \mathrm{NO}_{2} \mathrm{ArH}\right)$; ${ }^{13} \mathrm{C}$ NMR (100 MHz, CDCl 3 , gHSQC) 28.7 (C-8), 29.5 (C-8a), 37.2. 37.8 (C-5 and C7), $38.1\left(\mathrm{CH}_{2}\right), 40.1(\mathrm{C}-4), 40.2(\mathrm{C}-4 \mathrm{a}), 46.7\left(\mathrm{NCH}_{2}\right), 52.9(\mathrm{C}-1), 54.4(\mathrm{C}-3), 63.0$ $\left(\mathrm{CH}_{2} \mathrm{Ph}\right), 119.0\left(=\mathrm{CH}_{2}\right), 125.6,127.2,128.3,128.7,131.2,132.8,133.2,133.7,138.3$ ( $=\mathrm{CH}$ and Ar), 148.1 (C-ipso), 210.9 (C-6); HRMS (ESI-TOF): calcd for $\mathrm{C}_{28} \mathrm{H}_{32} \mathrm{~N}_{3} \mathrm{O}_{5} \mathrm{~S}$ $498.2057[\mathrm{M}+\mathrm{H}]^{+}$found 498.2056. 


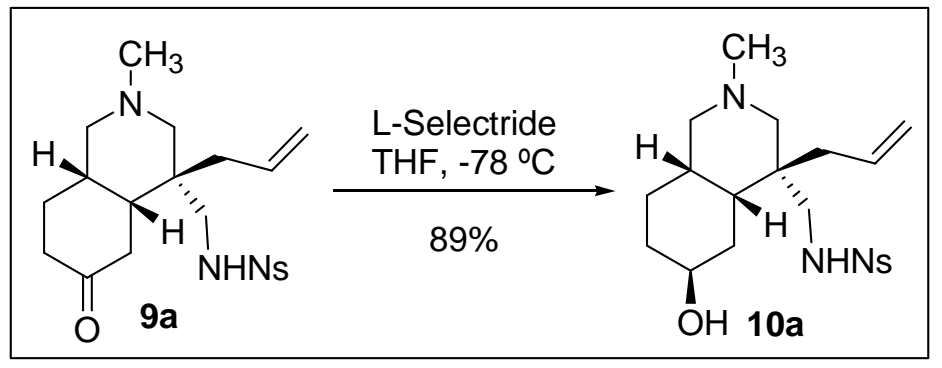

(4RS,4a $R S, 6 S R, 8 \mathrm{a} S R)-N$-(4-Allyl-6-hydroxy-2-methyldecahydroisoquinolin-4ylmethyl)-2-nitrobenzenesulfonamide (10a). L-Selectride (1 M in THF, $0.36 \mathrm{~mL}, 0.36$ mmol) was added to a cooled $\left(-78^{\circ} \mathrm{C}\right)$ solution of ketone $9 \mathrm{a}(100 \mathrm{mg}, 0.24 \mathrm{mmol})$ in THF $(1.1 \mathrm{~mL})$, and the mixture was stirred at this temperature for $2 \mathrm{~h}$. The reaction mixture was quenched by the addition of isopropanol $(0.55 \mathrm{~mL}), \mathrm{H}_{2} \mathrm{O}(0.55 \mathrm{~mL})$ and $\mathrm{SiO}_{2}(1 \mathrm{~g})$. After being stirred for $20 \mathrm{~min}$, the mixture was filtered with Celite and concentrated, and the residue was purified by cromatography $\left(\mathrm{Al}_{2} \mathrm{O}_{3}, \mathrm{CH}_{2} \mathrm{Cl}_{2} / \mathrm{MeOH}\right.$ 95:5) to give alcohol 10a as a yellow oil (90 mg, 89\%): IR ( $\mathrm{NaCl}) 3350,2930,2795$, 1542, 1361; ${ }^{1} \mathrm{H}$ NMR (400 MHz, $\mathrm{CDCl}_{3}$, gCOSY) 1.37 (dm, $J=13.6 \mathrm{~Hz}, 1 \mathrm{H}, \mathrm{H}-8 \mathrm{eq}$ ), 1.46-1.62 (m, 3H, H-5eq and H-7), 1.64 (td, $J=13.6,2.4 \mathrm{~Hz}, 1 \mathrm{H}, \mathrm{H}-5 \mathrm{ax}), 1.76$ (d, $J=$ 11.6 Hz, 1H, H-3, 1.88-2.06 (m, 2H, H-8 and H-8a), 2.10-2.19 (m, 2H, H-1 and H-4a), $2.32(\mathrm{~d}, J=11.6 \mathrm{~Hz}, 1 \mathrm{H}, \mathrm{H}-3), 2.42\left(\mathrm{~m}, 2 \mathrm{H}, \mathrm{H}-1\right.$ and $\left.\mathrm{CH}_{2}\right), 2.55(\mathrm{dd}, J=13.2,7.2 \mathrm{~Hz}, 1$ $\left.\mathrm{H}, \mathrm{CH}_{2}\right), 2.77,2.81\left(2 \mathrm{~d}, J=10.7 \mathrm{~Hz}, 1 \mathrm{H}\right.$ each, $\left.\mathrm{NCH}_{2}\right), 4.11\left(\mathrm{~m}, W_{1 / 2}=8.8 \mathrm{~Hz}, 1 \mathrm{H}, \mathrm{H}-\right.$ $6 \mathrm{eq}), 5.04\left(\mathrm{dd}, J=10,1.6 \mathrm{~Hz}, 1 \mathrm{H},=\mathrm{CH}_{2}\right), 5.12\left(\mathrm{dd}, J=17.2,1.6 \mathrm{~Hz}, 1 \mathrm{H},=\mathrm{CH}_{2}\right), 5.50$ $(\mathrm{b}, 1 \mathrm{H}, \mathrm{NH}), 5.75(\mathrm{~m}, 1 \mathrm{H},=\mathrm{CH}), 7.70,7.85,8.10(3 \mathrm{~m}, \mathrm{ArH}) ;{ }^{13} \mathrm{C}$ NMR $(100 \mathrm{MHz}$, $\mathrm{CDCl}_{3}$, gHSQC) 23.1 (C-8), 27.4 (C-7), 27.7 (C-5), 30.0 (C-4a), 31.3 (C-8a), 37.8 $\left(\mathrm{CH}_{2}\right), 39.5(\mathrm{C}-4), 46.6\left(\mathrm{CH}_{3}\right), 47.0\left(\mathrm{NCH}_{2}\right), 55.1(\mathrm{C}-1), 57.4(\mathrm{C}-3), 66.2(\mathrm{C}-6), 118.6$ $\left(=\mathrm{CH}_{2}\right), 125.4,131.2,132.7,133.1,133.5,133.8,134.3(=\mathrm{CH}$ and Ar); HRMS (ESITOF): calcd for $\mathrm{C}_{20} \mathrm{H}_{30} \mathrm{~N}_{3} \mathrm{O}_{5} \mathrm{~S} 424.1899[\mathrm{M}+\mathrm{H}]^{+}$found 424.1901 .

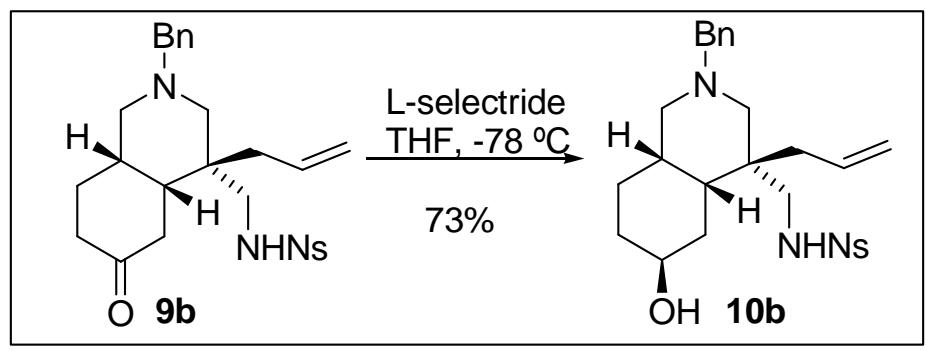

(4RS,4aRS,6SR,8aSR)-N-(4-Allyl-2-benzyl-6-hydroxydecahydroisoquinolin-4ylmethyl)-2-nitrobenzenesulfonamide (10b). Starting from ketone 9b (0.51 g, 0.9 mmol), operating as above, chromatography $\left(\mathrm{Al}_{2} \mathrm{O}_{3}, \mathrm{CH}_{2} \mathrm{Cl}_{2}\right.$ to $\mathrm{CH}_{2} \mathrm{Cl}_{2} / \mathrm{MeOH}$ 98:2) gave alcohol 10b (365 mg, 73\%): IR(NaCl) 3357, 3350, 2930, 2807, 1541, 1358; ${ }^{1} \mathrm{H}$ NMR (400 MHz, CDCl 3 , gCOSY) 1.27-1.33 (m, 1H, H-8), 1.50-1.58 (m, 3H, H-5, H7), $1.63-1.70(\mathrm{~m}, 1 \mathrm{H}, \mathrm{H}-5), 1.78$ (d, $J=11.2 \mathrm{~Hz}, 1 \mathrm{H}, \mathrm{H}-3), 1.90-2.00(\mathrm{~m}, 1 \mathrm{H}, \mathrm{H}-8), 2.04$ (da, $J=12.8 \mathrm{~Hz}, 1 \mathrm{H}, \mathrm{H}-8 \mathrm{a}$ ), 2.22-2.29 (m, 3H, H-1, H-3, H-4a), 2.37 (dd, $J=14.2,8.2$ $\left.\mathrm{Hz}, 1 \mathrm{H}, \mathrm{CH}_{2}\right), 2.47(\mathrm{dd}, J=19,11,8 \mathrm{~Hz}, 1 \mathrm{H}, \mathrm{H}-1), 2.65-2.73\left(\mathrm{~m}, 2 \mathrm{H}, \mathrm{NCH}_{2}, \mathrm{CH}_{2}\right), 2.77$ $\left(\mathrm{dd}, J=11.8,6.6 \mathrm{~Hz}, 1 \mathrm{H}, \mathrm{NCH}_{2}\right), 3.35$ and $3.48\left(2 \mathrm{~d}, J=13.4 \mathrm{~Hz}, 1 \mathrm{H}\right.$ each, $\left.\mathrm{CH}_{2} \mathrm{Ph}\right)$, $4.12\left(\mathrm{brs}, W_{1 / 2}=9 \mathrm{~Hz}, 1 \mathrm{H}, \mathrm{H}-6\right), 4.94\left(\mathrm{dd}, J=10 \mathrm{~Hz}, 1.6,1 \mathrm{H},=\mathrm{CH}_{2}\right), 5.06(\mathrm{~d}, J=17.2$ $\left.\mathrm{Hz}, 1 \mathrm{H},=\mathrm{CH}_{2}\right), 5.42(\mathrm{tbr}, J=5.8 \mathrm{~Hz}, 1 \mathrm{H}, \mathrm{NH}), 5.62(\mathrm{~m}, 1 \mathrm{H},=\mathrm{CH}), 7.22-7.30(\mathrm{~m}, 5 \mathrm{H}$, 
ArH), 7.71, 7.83 and $8.06\left(3 \mathrm{~m}, 4 \mathrm{H}, \mathrm{NO}_{2} \mathrm{ArH}\right) ;{ }^{13} \mathrm{C} \mathrm{NMR}\left(100 \mathrm{MHz}, \mathrm{CDCl}_{3}\right.$, gHSQC) 23.1 (C-8), 27.3 (C-7), 27.7 (C-5), 30.0 (C-4a), 32.1 (C-8a), $37.9\left(\mathrm{CH}_{2}\right), 39.4$ (C-4), $46.7\left(\mathrm{NCH}_{2}\right), 53.1(\mathrm{C}-1), 54.3(\mathrm{C}-3), 63.1\left(\mathrm{CH}_{2} \mathrm{Ph}\right), 66.3(\mathrm{C}-6), 118.4\left(=\mathrm{CH}_{2}\right), 127.0$, 128.1, 128.3 and 128.7 (Ar), 125.4, 131.2, 132.7, 133.5, $134.0\left(=\mathrm{CH}, \mathrm{NO}_{2} \mathrm{Ar}\right), 138.8$ and 148.1 (C-ipso); HRMS (ESI-TOF): calcd for $\mathrm{C}_{26} \mathrm{H}_{34} \mathrm{~N}_{3} \mathrm{O}_{5} \mathrm{~S} 500.2199[\mathrm{M}+\mathrm{H}]^{+}$found 500.2214 .

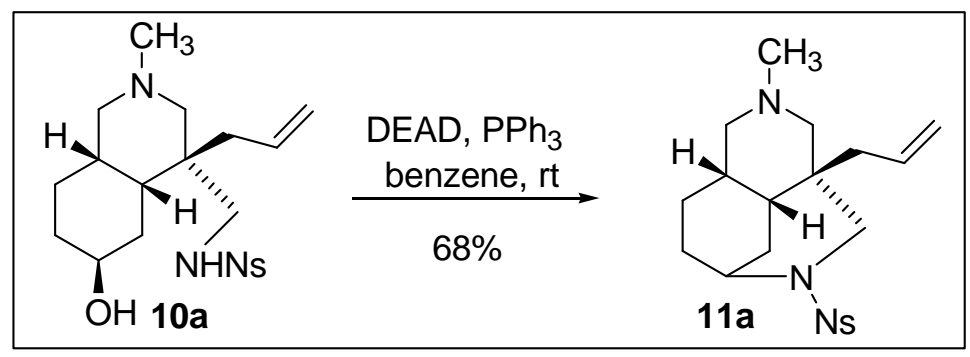

(4RS,4aRS,6RS,8aSR)-4-Allyl-3-methyl-9-(2-nitrophenylsulphonyl)perhydro-6,4(iminomethano)isoquinoline (11a). To a stirred solution of 2-nitrobenzenesulfonamide 10a (90 mg, $0.21 \mathrm{mmol})$ and $\mathrm{PPh}_{3}(124 \mathrm{mg}, 0.53 \mathrm{mmol})$ in benzene $(2 \mathrm{~mL})$ was slowly added DEAD $(84 \mu \mathrm{L}, 0.53 \mathrm{mmol})$ at rt. After stirring overnight, the reaction mixture was concentrated, and the residue was purified by chromatography $\left(\mathrm{Al}_{2} \mathrm{O}_{3}\right.$, hexane to hexane $/ \mathrm{CH}_{2} \mathrm{Cl}_{2}$ 95:5) to give the tricyclic derivative $11 \mathrm{a}(60 \mathrm{mg}, 0.15 \mathrm{mmol}, 68 \%)$ as a yellow oil: $\mathrm{IR}(\mathrm{NaCl}) 3075,2930,2782,1544,1492,1372 ;{ }^{1} \mathrm{H} \mathrm{NMR}\left(400 \mathrm{MHz}, \mathrm{CDCl}_{3}\right.$, gCOSY) $1.38-1.52(\mathrm{~m}, 2 \mathrm{H}, \mathrm{H}-7, \mathrm{H}-4 \mathrm{a}), 1.52-1.70\left(\mathrm{~m}, 3 \mathrm{H}, \mathrm{H}-8, \mathrm{H}-5, \mathrm{CH}_{2}\right), 1.77$ (d, $J=$ $10 \mathrm{~Hz}, 1 \mathrm{H}, \mathrm{H}-3), 1.80-1.98$ (m, 2H, H-1, H-3), 2.11 (dd, $J=13.6,7.4 \mathrm{~Hz}, 1 \mathrm{H}, \mathrm{CH}_{2}$ ), 2.14-2.17 (m, 2H, H-8, H-7), 2.18 (s, 3H, CH $), 2.36$ (da, $J=11.2 \mathrm{~Hz}, 1 \mathrm{H}, \mathrm{H}-3), 2.54$ $(\mathrm{d}, J=10.2 \mathrm{~Hz}, 1 \mathrm{H}, \mathrm{H}-1), 3.23$ and $3.85(2 \mathrm{~d}, J=13.2 \mathrm{~Hz}, 1 \mathrm{H}$ each, H-10), 4.14 (brs, $\left.W_{1 / 2}=10 \mathrm{~Hz}, 1 \mathrm{H}, \mathrm{H}-6\right), 4.72\left(\mathrm{dd}, J=17,2.2 \mathrm{~Hz}, 1 \mathrm{H},=\mathrm{CH}_{2}\right), 4.92(\mathrm{dd}, J=10.2,2.2 \mathrm{~Hz}$, $\left.1 \mathrm{H},=\mathrm{CH}_{2}\right), 5.59(\mathrm{~m}, 1 \mathrm{H},=\mathrm{CH}), 7.70$ i $8,05\left(2 \mathrm{~m}, 4 \mathrm{H}, \mathrm{NO}_{2} \mathrm{ArH}\right) ;{ }^{13} \mathrm{C} \mathrm{NMR}(100 \mathrm{MHz}$, $\mathrm{CDCl}_{3}$, gHSQC) 24.9 (C-8), 28.7 (C-7), 30.5 (C-5), 34.5 (C-8a), 34.6 (C-4a), 35.8 (C4), $40.9\left(\mathrm{CH}_{2}\right), 46.6\left(\mathrm{CH}_{3}\right), 47.4(\mathrm{C}-6), 49.3(\mathrm{C}-10), 61.3(\mathrm{C}-1), 63.4(\mathrm{C}-3), 118.5$ $\left(=\mathrm{CH}_{2}\right), 124.2,131.1,131,4,133.0,133.1\left(=\mathrm{CH}, \mathrm{NO}_{2} \mathrm{Ar}\right), 133.6$ and 147.9 (C-ipso). HRMS (ESI-TOF): calcd for $\mathrm{C}_{20} \mathrm{H}_{28} \mathrm{~N}_{3} \mathrm{O}_{4} \mathrm{~S} 406.1795[\mathrm{M}+\mathrm{H}]^{+}$found 406.1793 . 
H1 / s2pul / Gemini-300A

cdc13/Temp: Ambient /N reg: PCB171105180957

Usuari: san / Mostra: lp835c

Nom: LAURA PALOMA ROMEU

Data:17/11/05/ Sist automatic

Pulse Sequence: s2pul

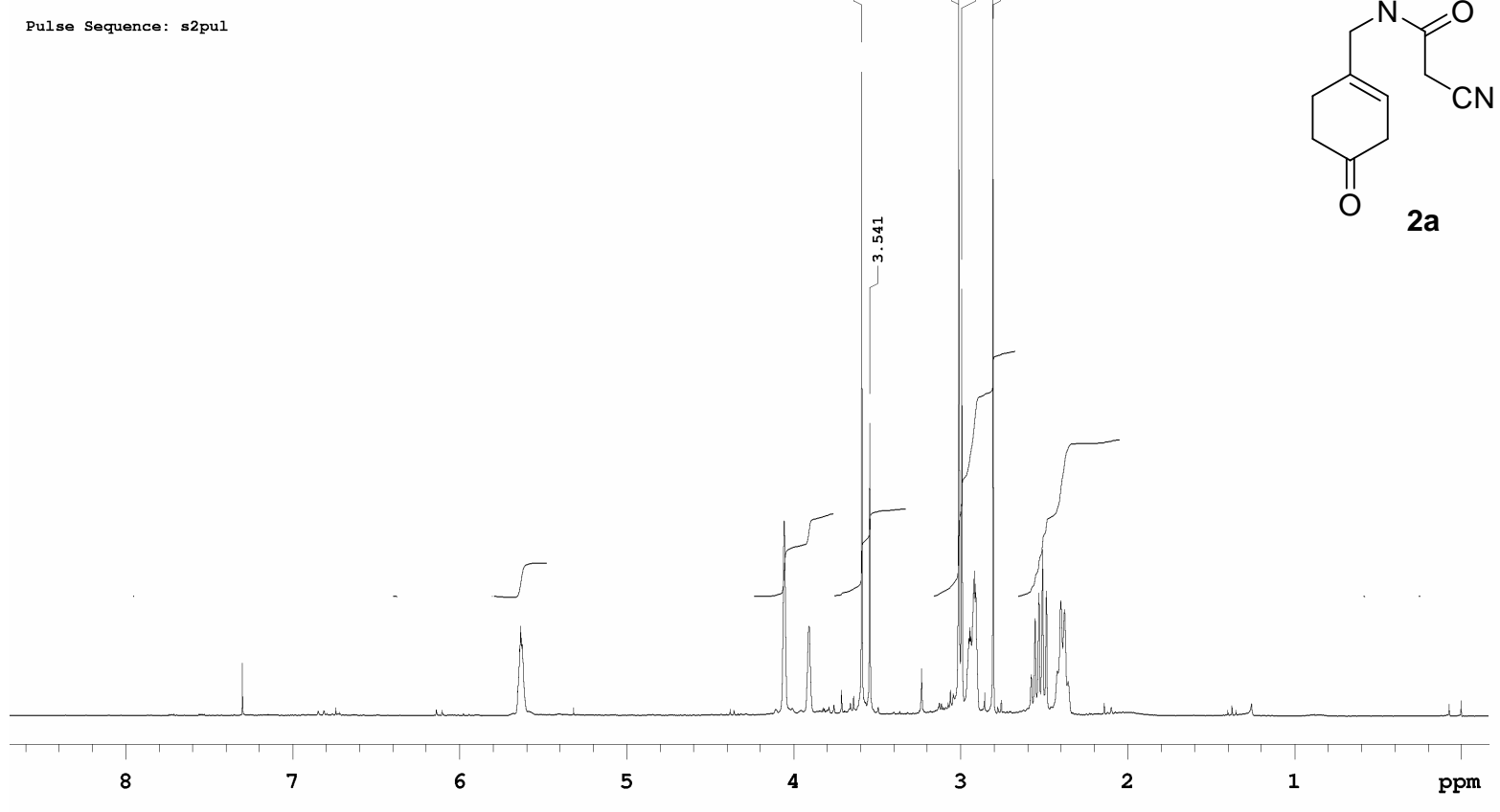

C13 / s2pul / Gemini-300A

cdc13/Temp: Ambient /N reg: PCB171105180957

Nom: LAURA PALOMA ROMEU

Data:17/11/05/Sist automatic

Pulse Sequence: s2pul
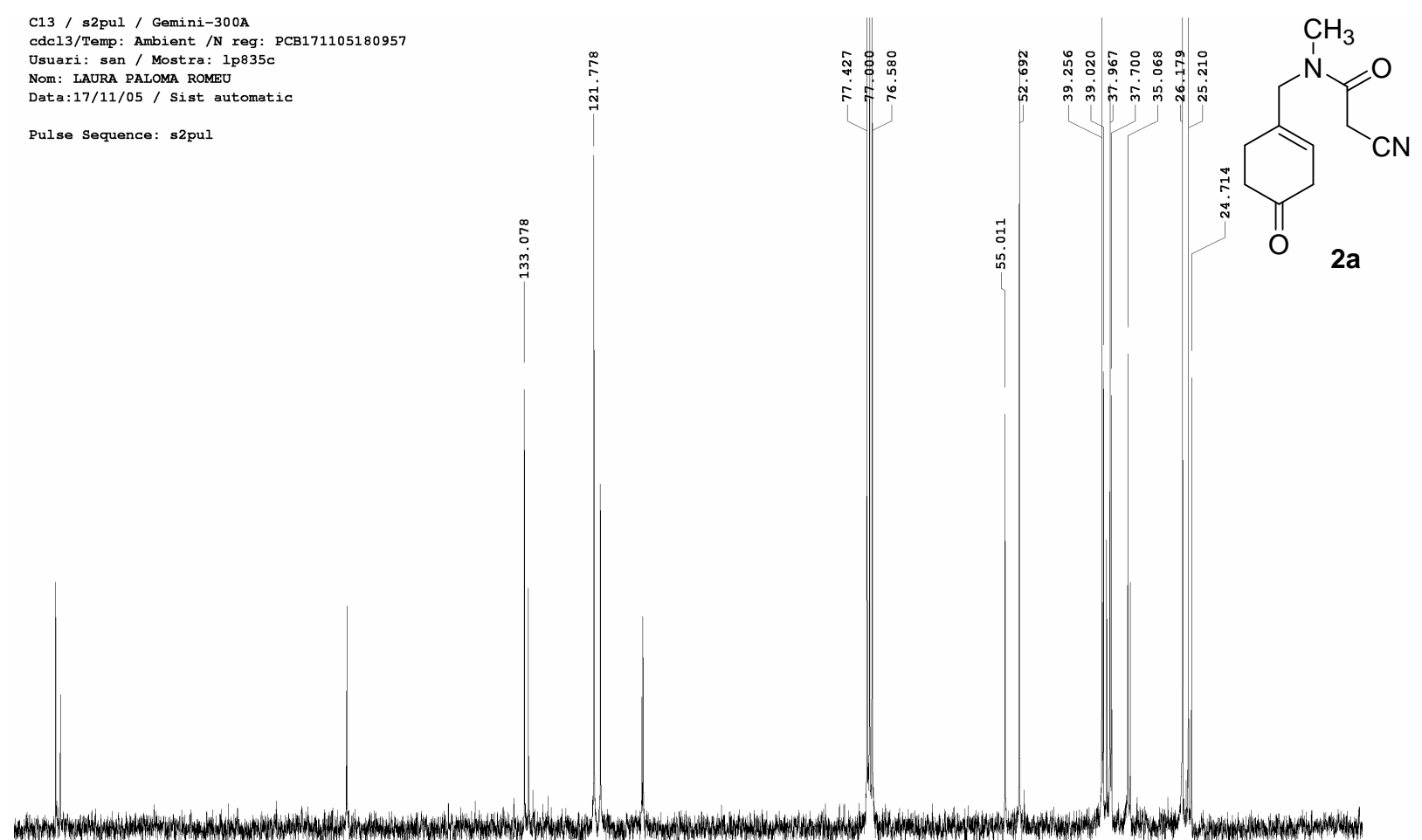

200

180

160

140

120

100

80

60

40

20

ppm 
cdc13/Temp: Ambient /N reg: PCB171105180957 Usuari: san / Mostra: 1p835c

Data:17/11/05/ Sist automatic

Pulse Sequence: $\cos Y$<smiles>CN(CC1=CCC(=O)CC1)C(=O)CC#N</smiles>

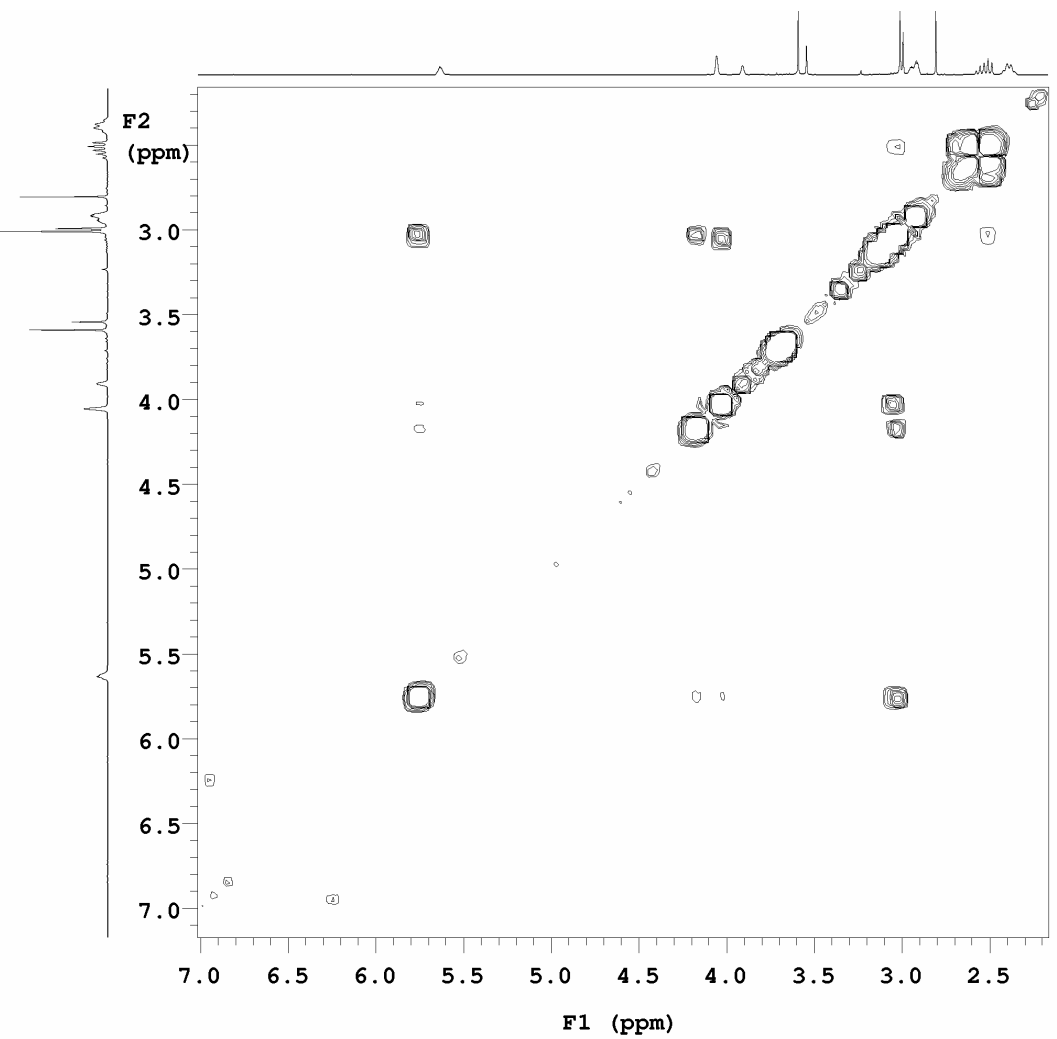


H1 / s2pul / Mercury-400 qui

cdc13/Temp: 25C /N reg: M40005-221105164303

Nom: M. MAR BORREGAN PRATS

Data: 22/11/05/ Sist automatic

Pulse Sequence: s2pul

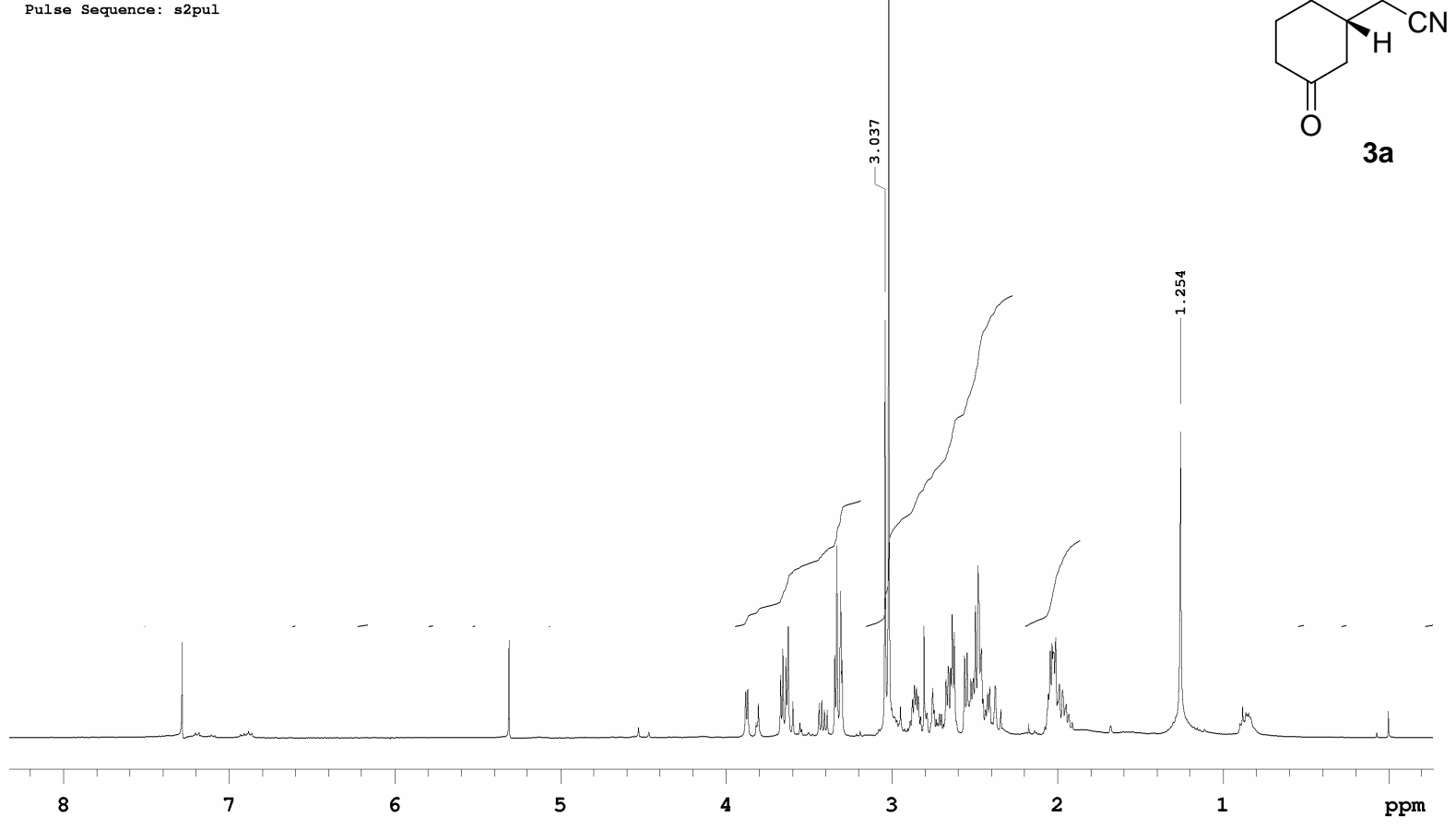

C13 / s2pul / Mercury-400_qui

$005-221105164303$

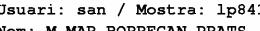

NOM: M.MAR BORREGAN PAAT

ㅇำ

Pulse Sequence: s2pul<smiles>CN1C[C@H]2CCC(=O)C[C@H]2C(C#N)C1=O</smiles>

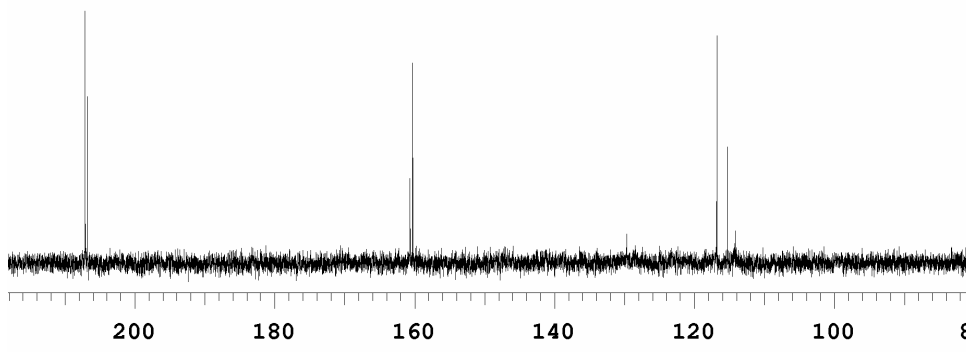

80

60

40

20

ppm 
H1 / gCosY / Mercury-400_qui cdc13/Temp: $25 \mathrm{C} / \mathrm{N}$ reg: M40005-221105164303 Usuarl: san / Mostra: 1pad

NOm: M.MAR BORREGAN PRATS
Data:23/11/05/ Sist automatic

Pulse Sequence: gcosy<smiles>CN1C[C@H]2CCC(=O)C[C@H]2C(C#N)C1=O</smiles>

$3 a$

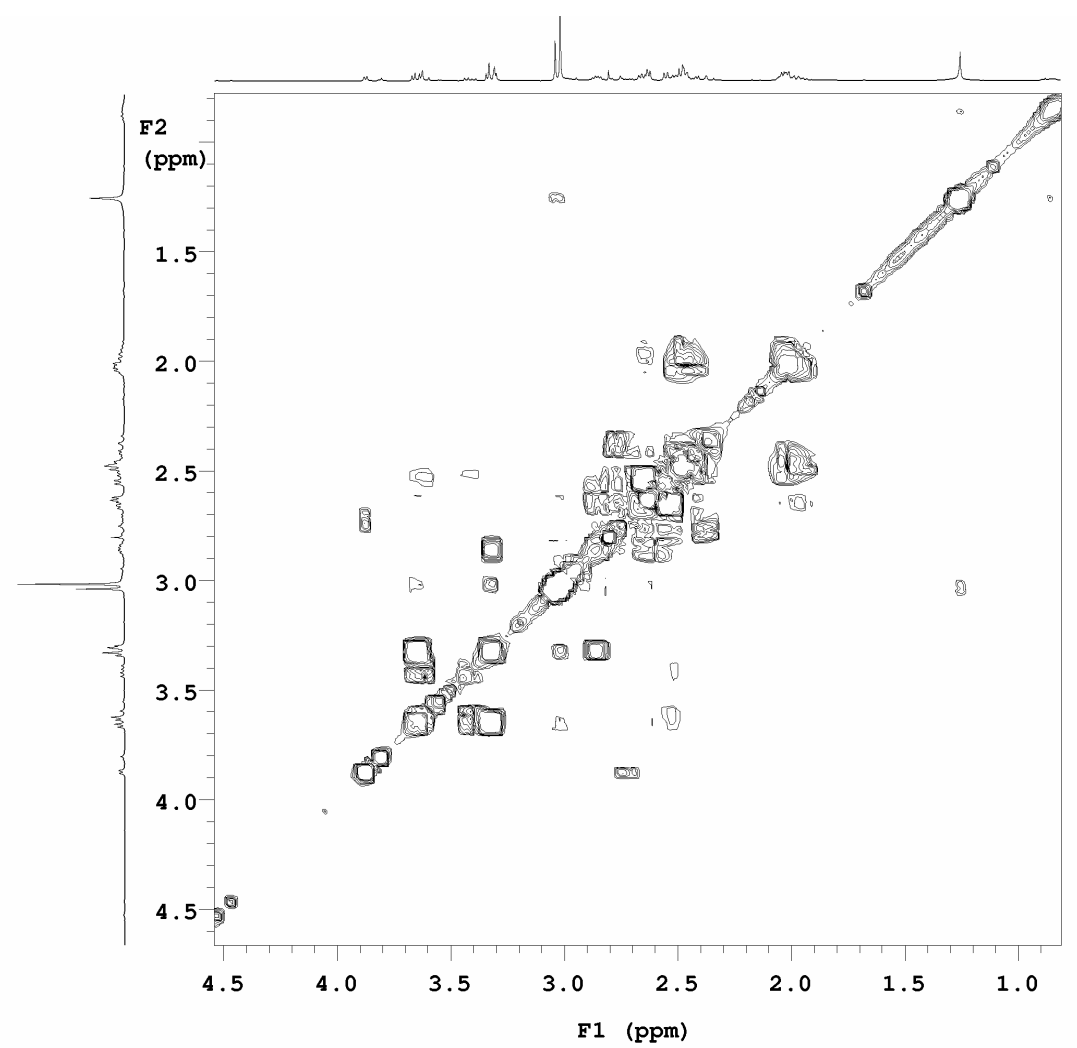

H1 / gHSQC / Mercury-400_qui

cdc13/Temp: 25C /N reg: M40005-221105164303

Usuari: san / Mostra: 1 p841

NOM: M.MAR BORREGAN PRATS

Data:23/11/05 / Sist automatic

Pulse Sequence: gHSQC<smiles>CN1C[C@H]2CCC(=O)C[C@H]2C(C#N)C1=O</smiles>

3a

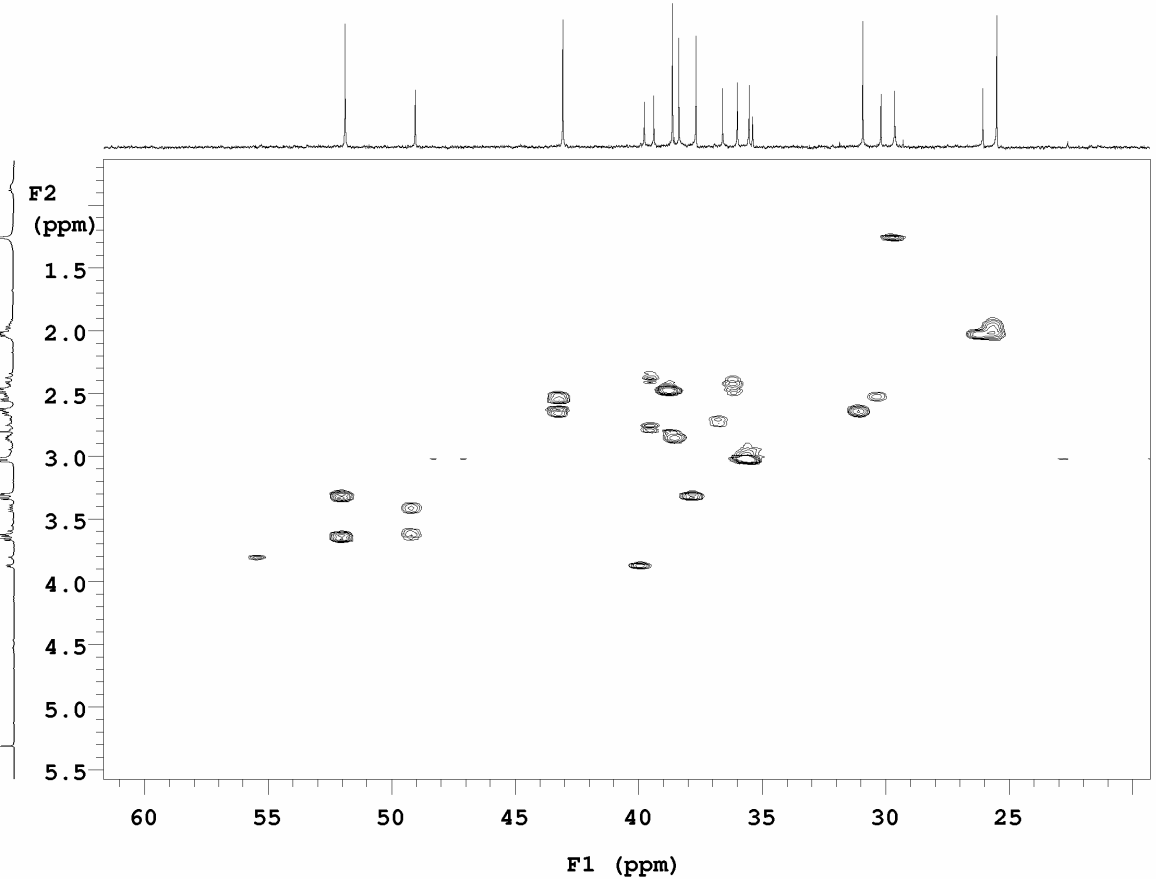




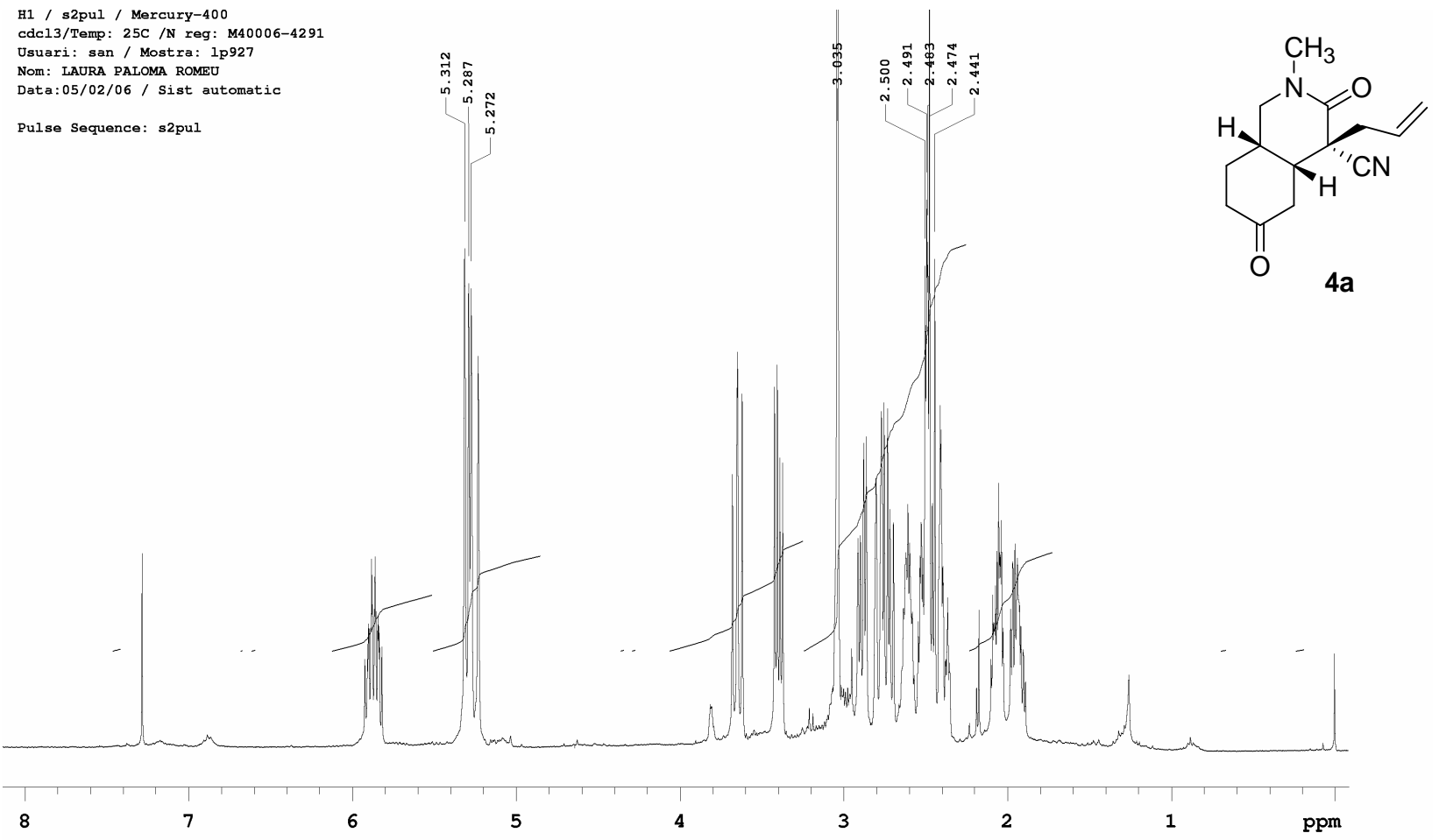

C13 / s2pul / Mercury-400

cdc13/Temp: $25 \mathrm{C} / \mathrm{N}$ reg: M40006-4291

Usuari: san / Mostra: 1p927

automatic

Pulse Sequence: s2pul
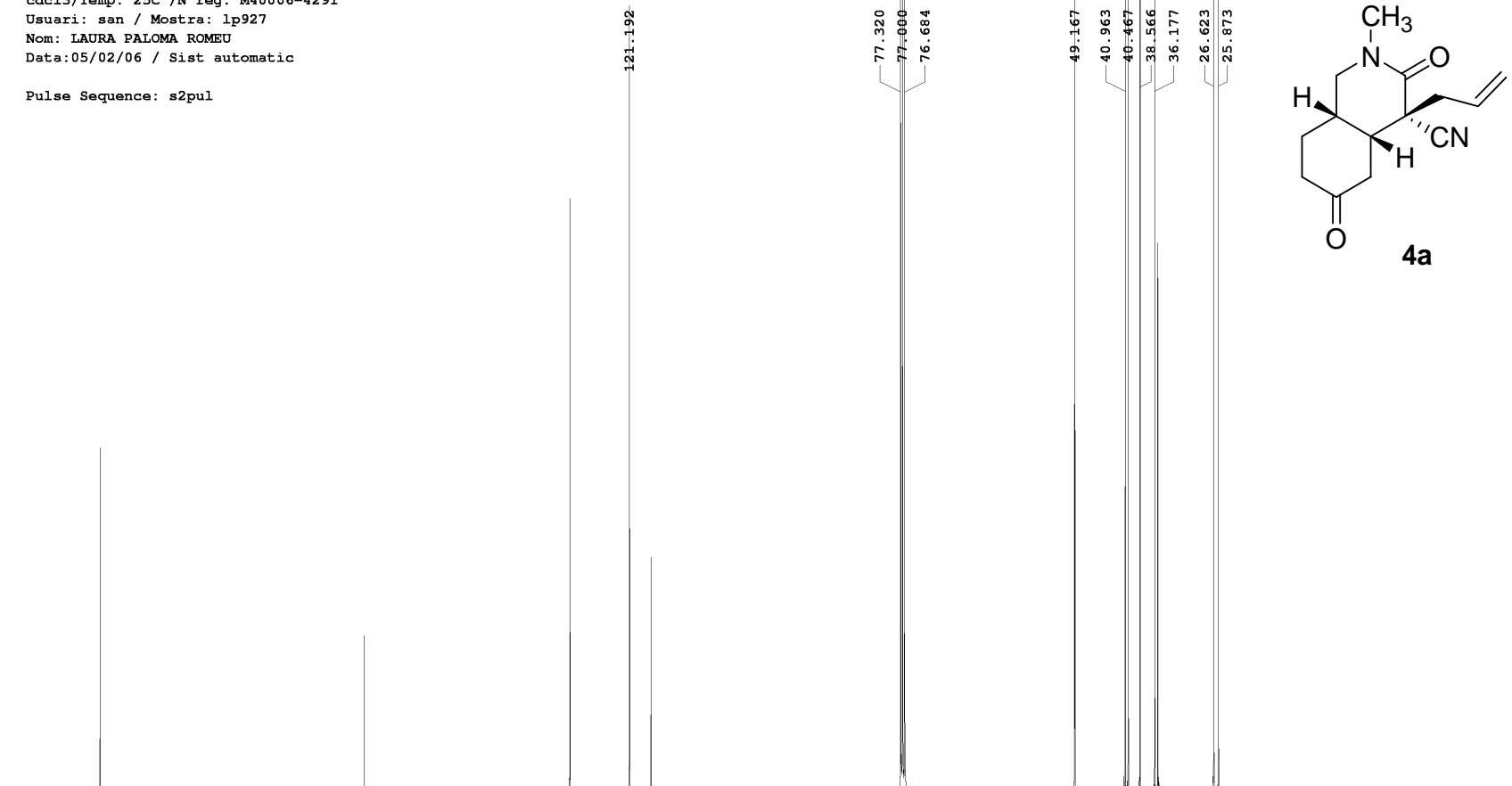

$4 a$

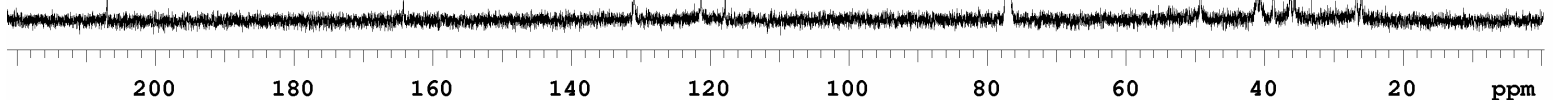


H1 / COSY / Gemini-300A

cdc13/Temp: $25 \mathrm{C} / \mathrm{N}$ reg: D-PCB06030718440

Usuari: san / Mostra: 1p1499

Data: 06/03/07/ Sist automatic

Pulse Sequence: CosY

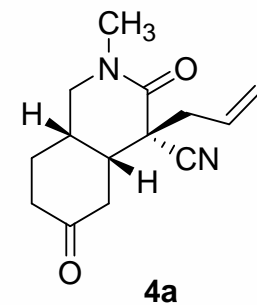

$4 a$

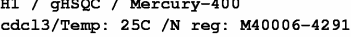

Usuari: san / Mostra: 1p927

Data: 05/02/06 / Sist automat

Pulse Sequence: gHSQC<smiles>C=CC[C@]1(C#N)C(=O)N(C)C[C@H]2CCC(=O)C[C@H]21</smiles>

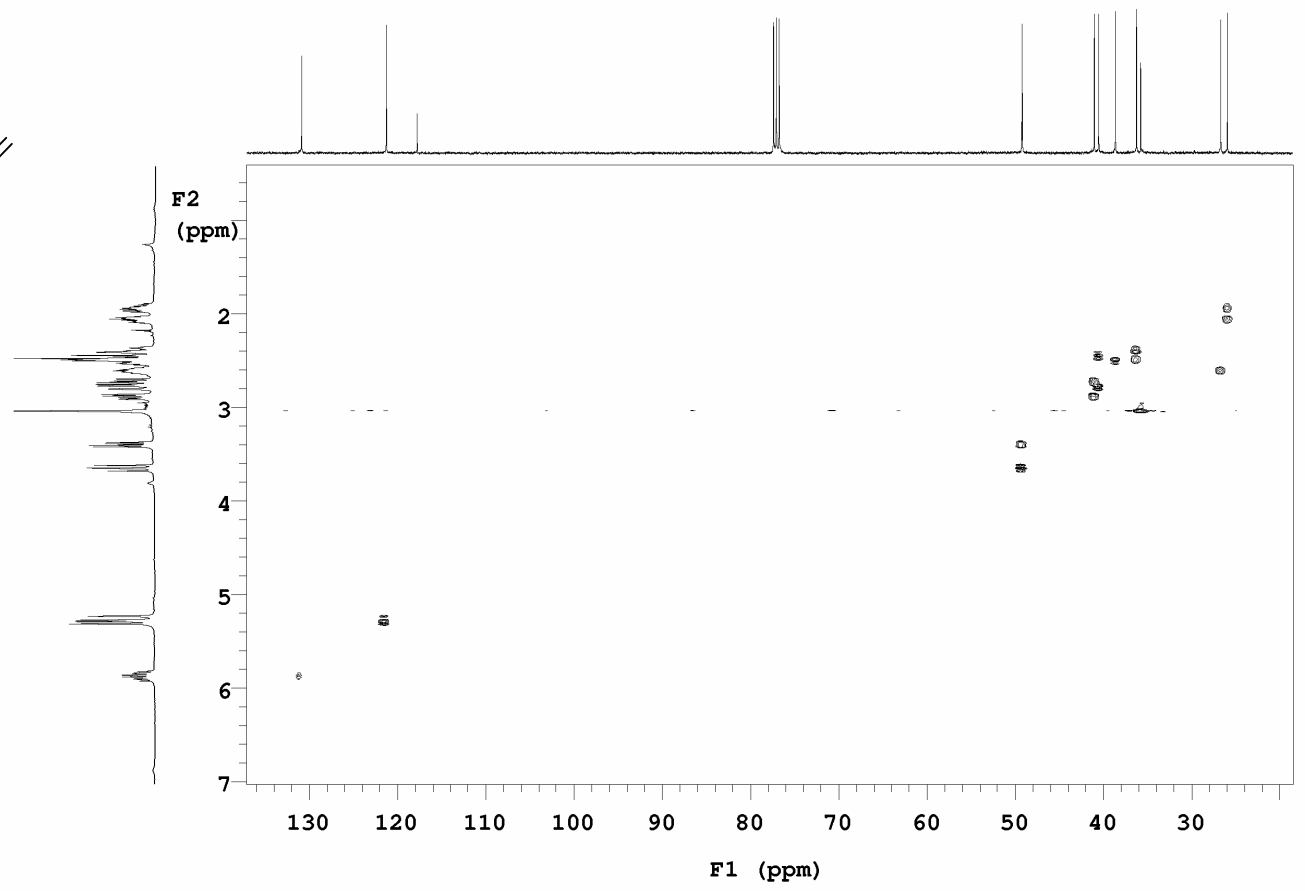




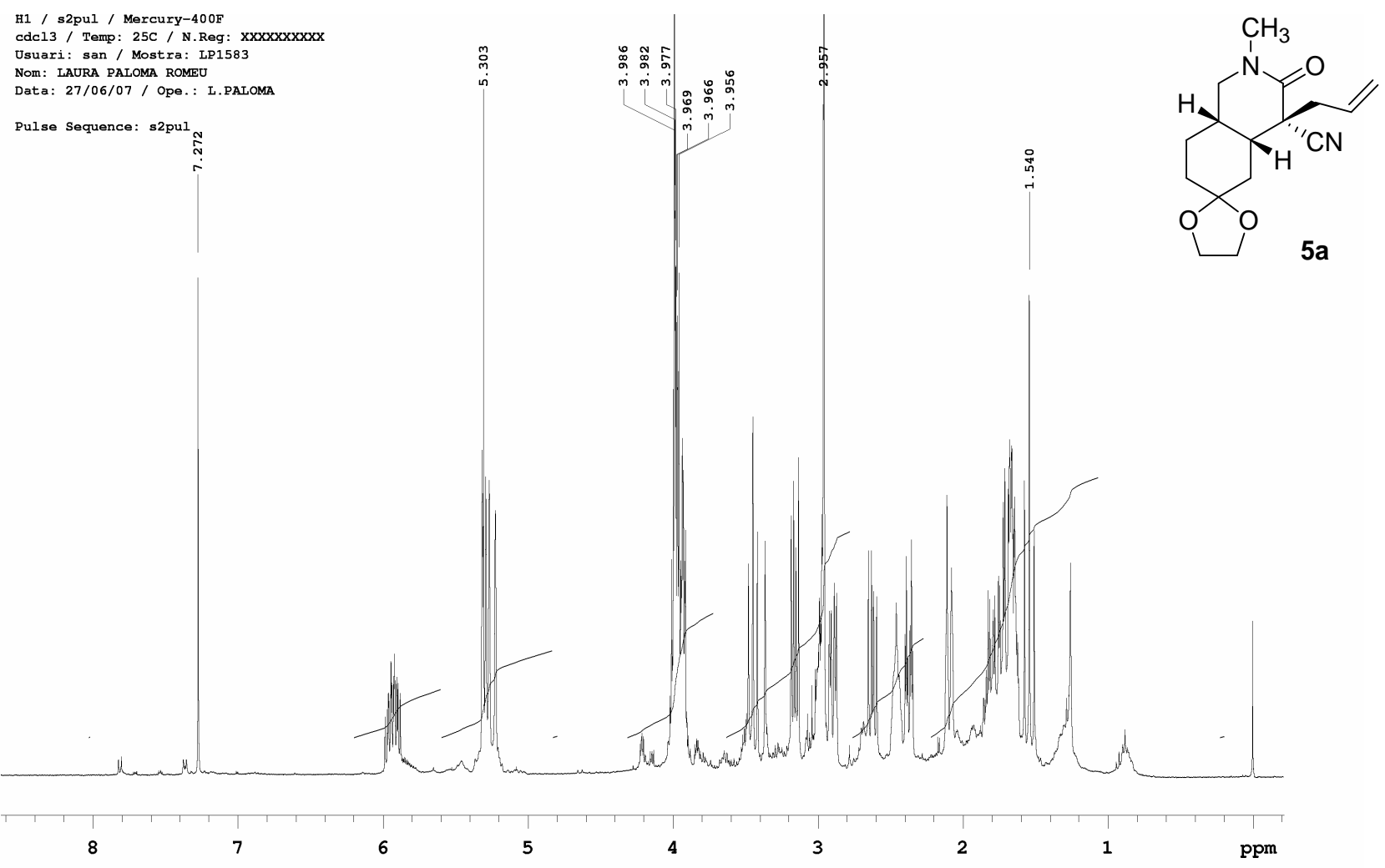

C13 / s2pul / Mercury-400 qui

cdc13/Temp: 25C /N reg: M40006-090206164641 Usuari: san / Mostra: 1p94

Data: $10 / 02 / 06 /$ Sist automatic

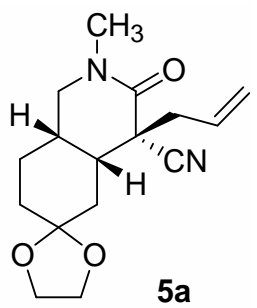

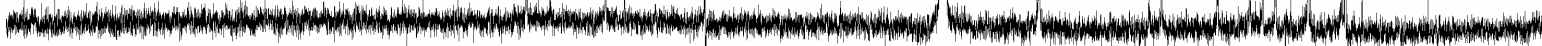


H1 / gCosY / Mercury-400F

Temp: 25C / N.Reg: $x x x x x x x x x x$

Usuri

Data: 27/06/07/ Ope.: L. PALOMA

Pulse Sequence: gcosy

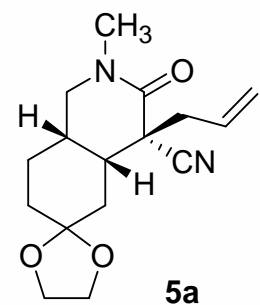

$5 a$

H1 / gHSQC / Mercury-400F

N. Reg: $x x x x x x x x x x$

Nom: LAURA PALOMA ROMEU

Data: 27/06/07 / Ope.: L.PALOMA

Pulse Sequence: gHSQC

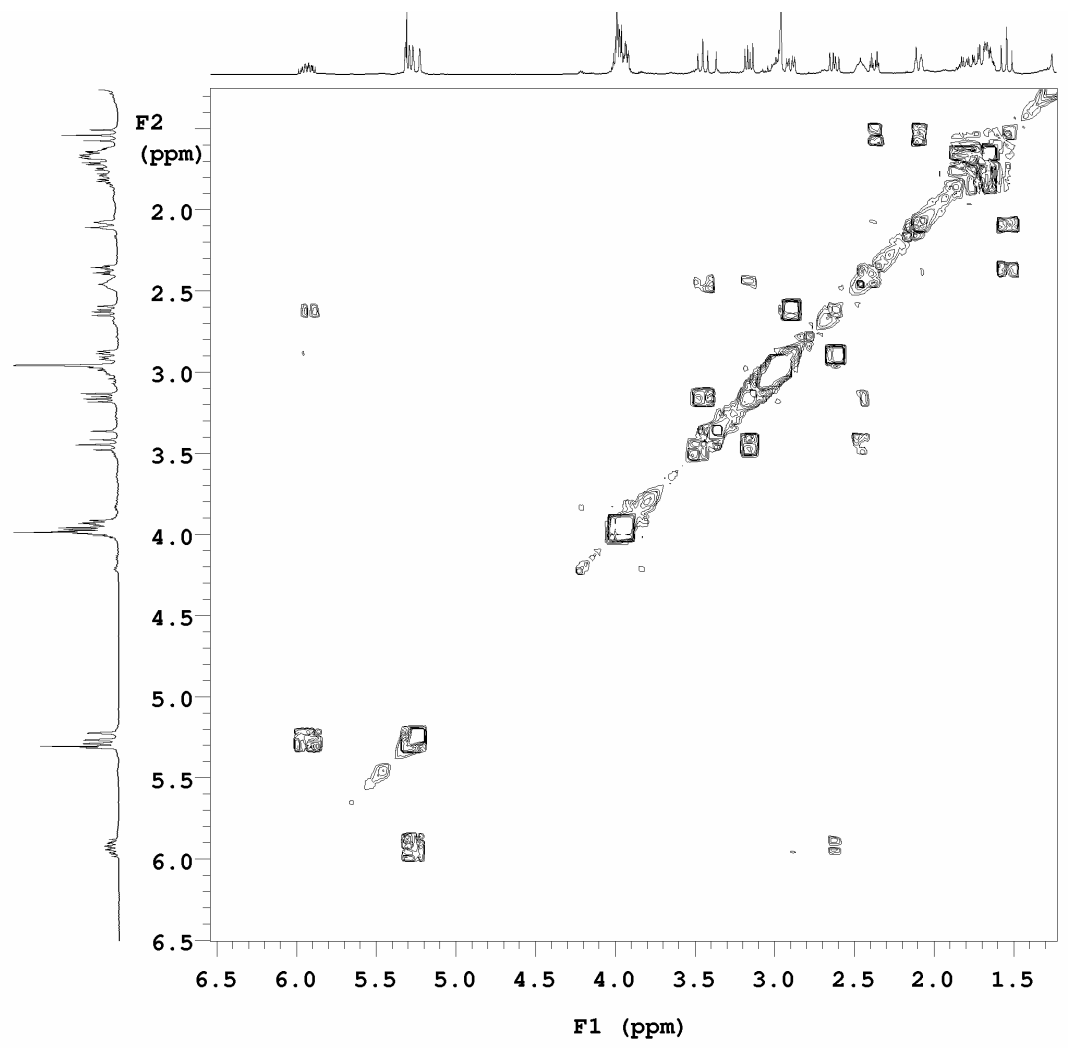

F1 (ppm)

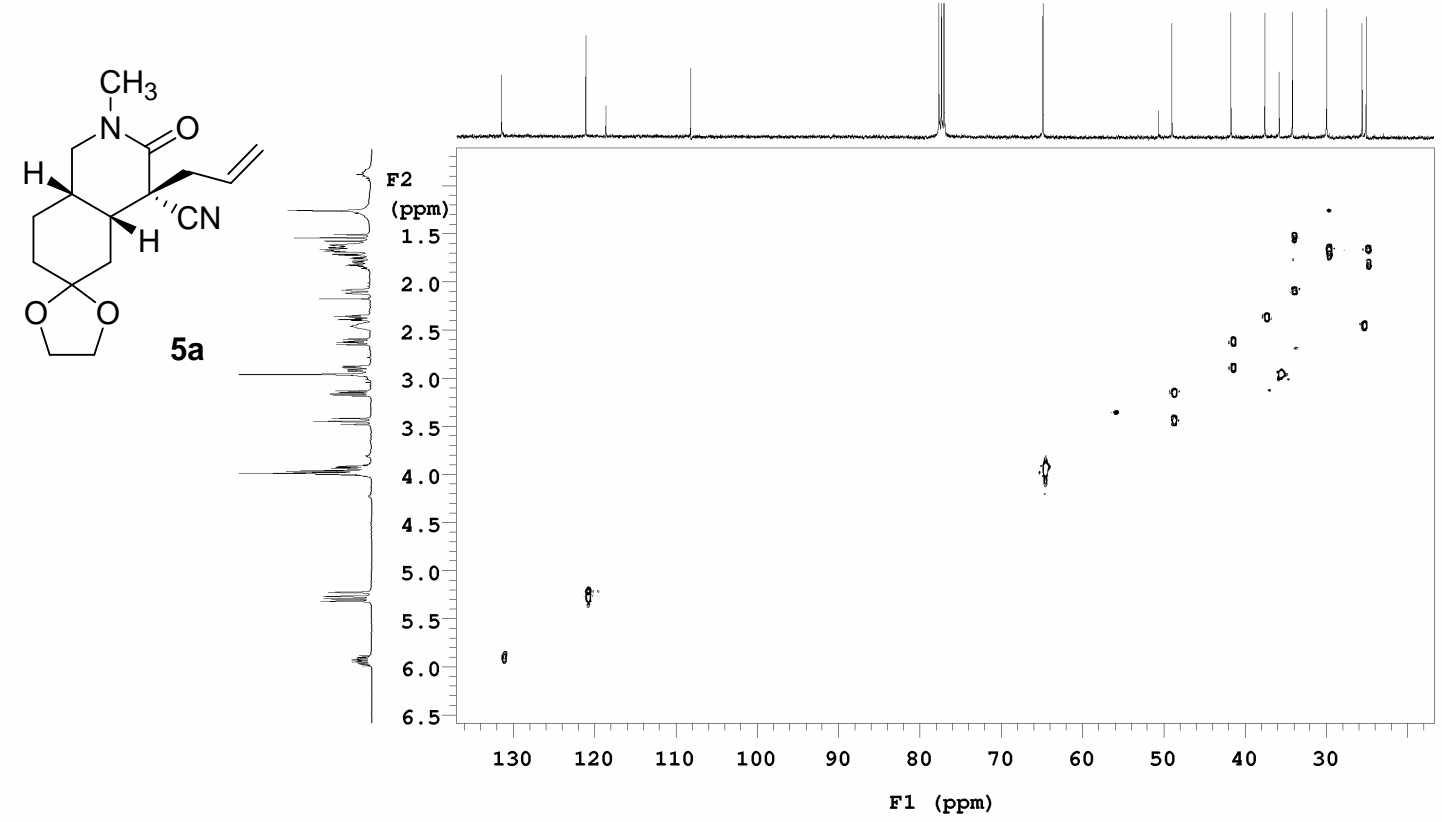


H1 / s2pul / Mercury-400_qui

cdc13/Temp: 25C /N reg: M40006-26050618035

Usuari: san / Mostra: 1palc13f1

Data:27/05/06/ Sist automatic

Pulse Sequence: s2pul
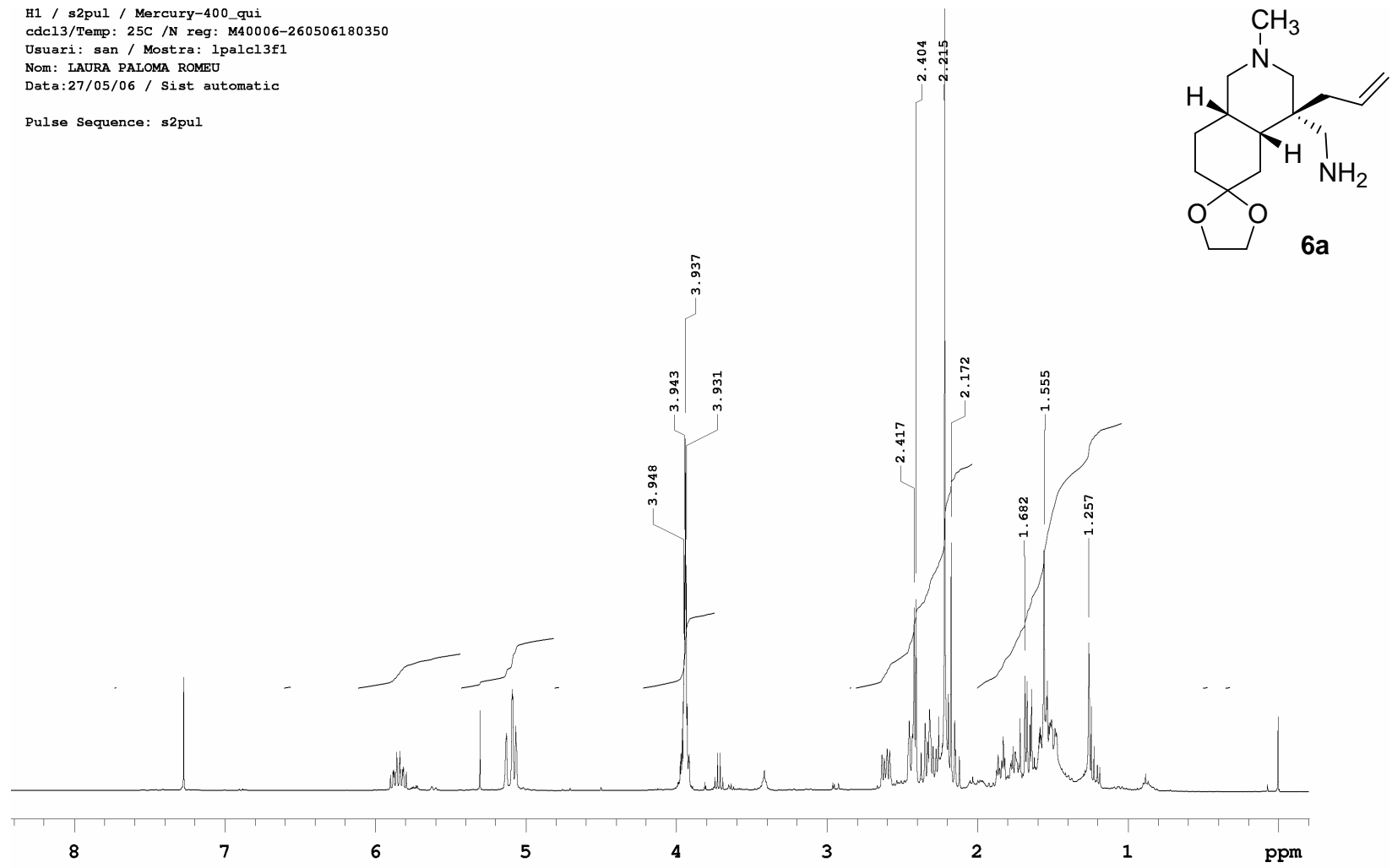

C13 / s2pul / Mercury-400_qui

cdc13/Temp: 25C /N reg: M40006-26050618035

Usuari: san / Mostra: 1palc13f1

Nom: LAURA PALOMA ROMLO

มี

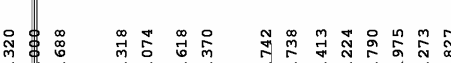

Pulse Sequence: s2pul

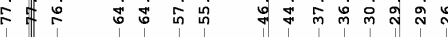

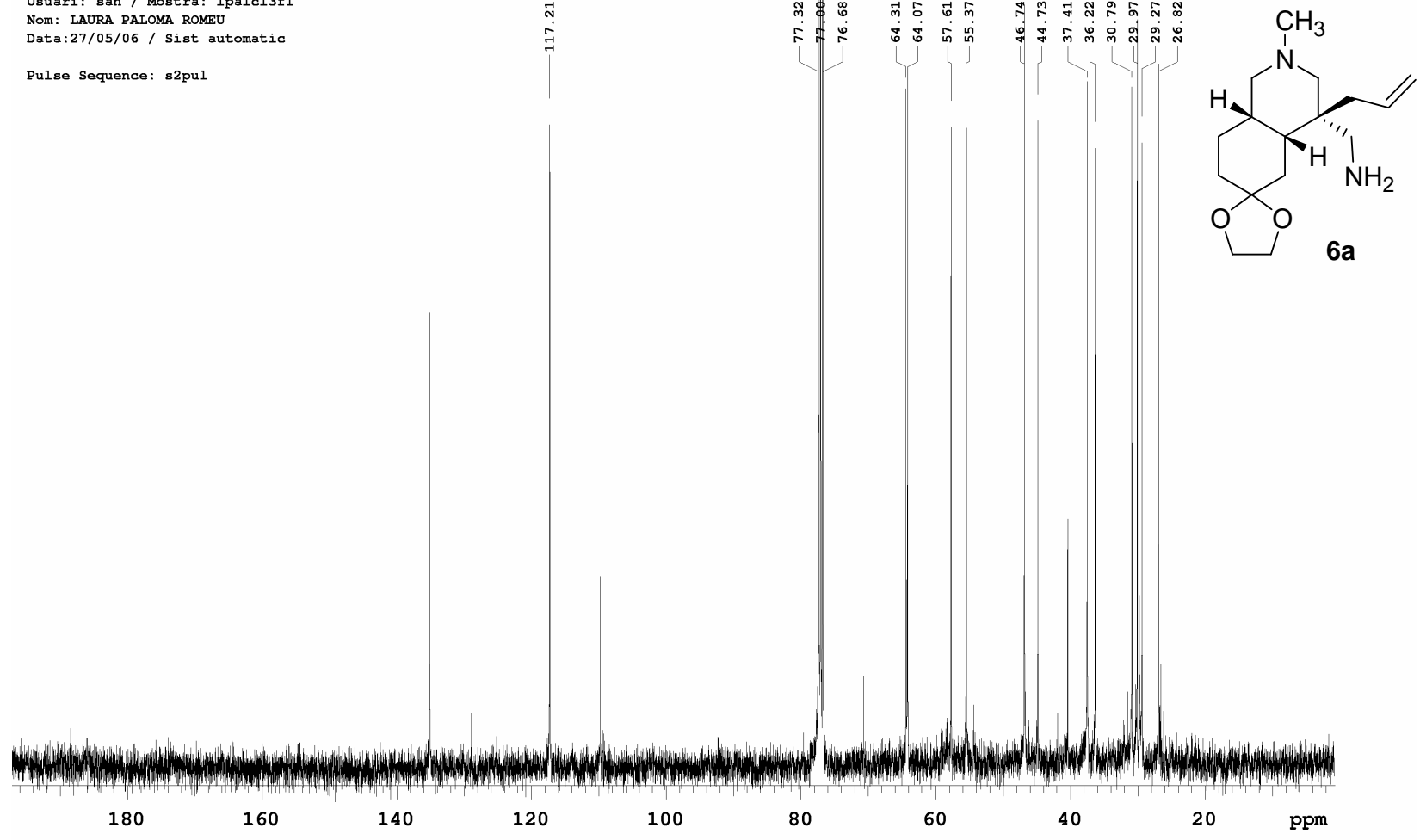


vnmrs 400

camrs $25 \mathrm{C} / \mathrm{N}$. reg: 2103-2006 Laura Paloma/san data : 02-06-2006

Pulse Sequence: gcosY<smiles>C=CC[C@]1(CN)CN(C)C[C@@H]2CCC3(C[C@H]21)OCCO3</smiles>

$6 a$

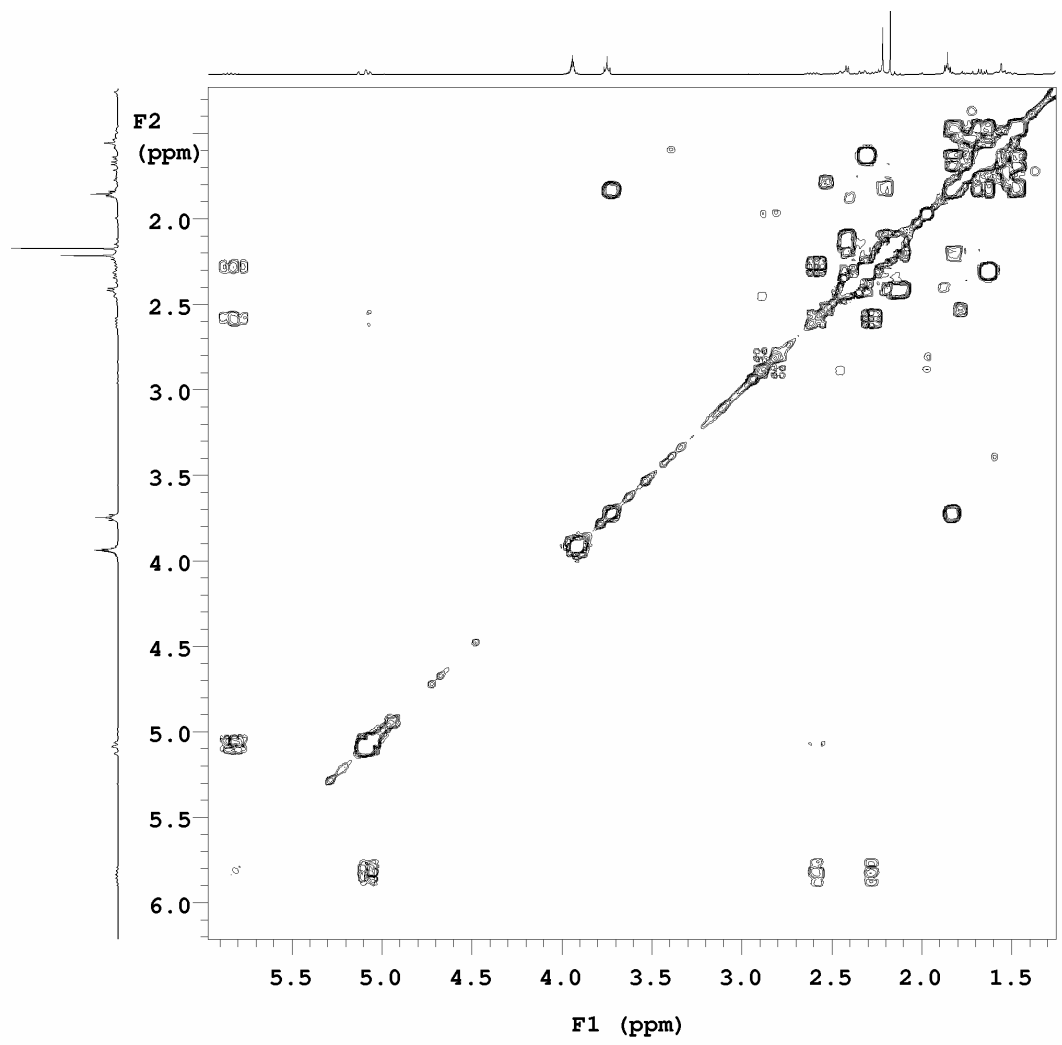

H1 / gHSQC / Mercury-400_qui

cdc13/Temp: 25C /N reg: M40006-260506180350

Usuari: san / Mostra: lpalc13f1

Data: 27/05/06/ Sist autongtic

Pulse Sequence: gHSOC<smiles>C=CC[C@]1(CN)CN(C)C[C@H]2CCC3(C[C@H]21)OCCO3</smiles><smiles>CCCCCCC(C)C</smiles>

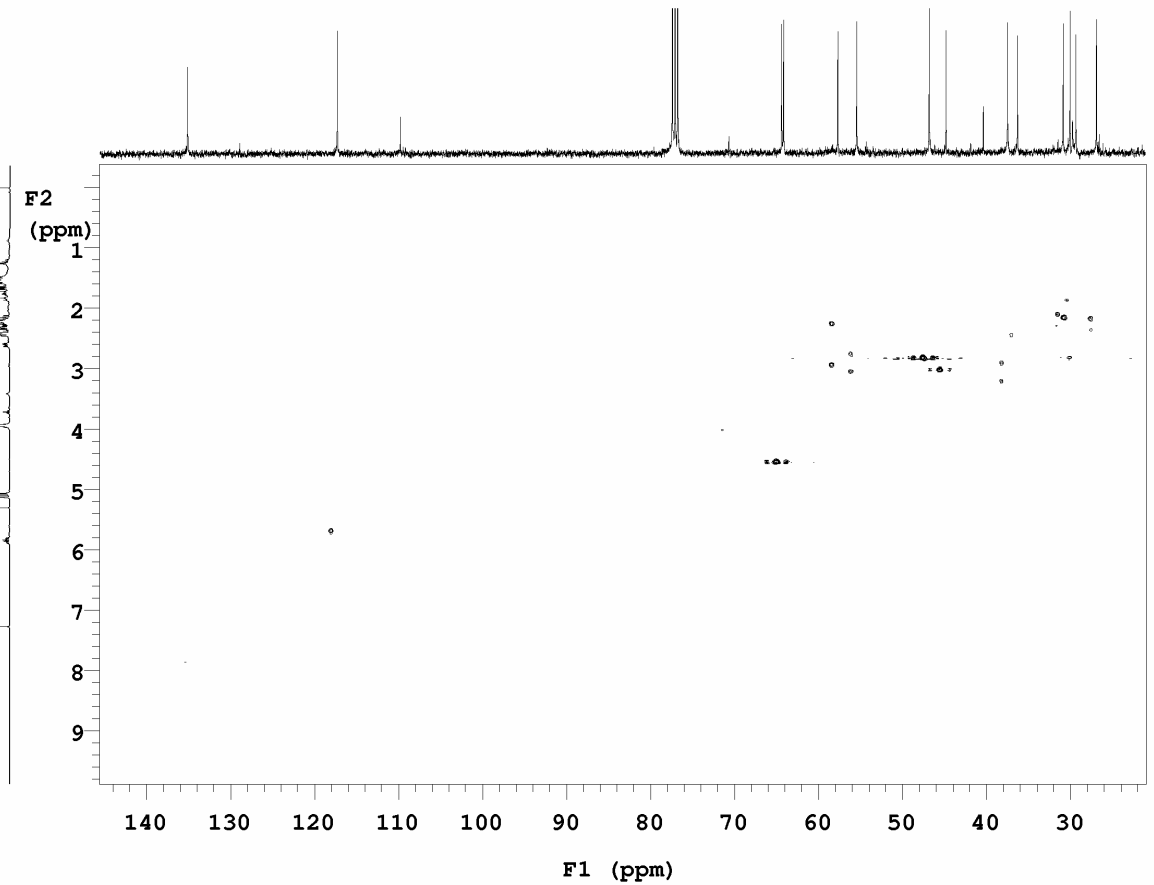




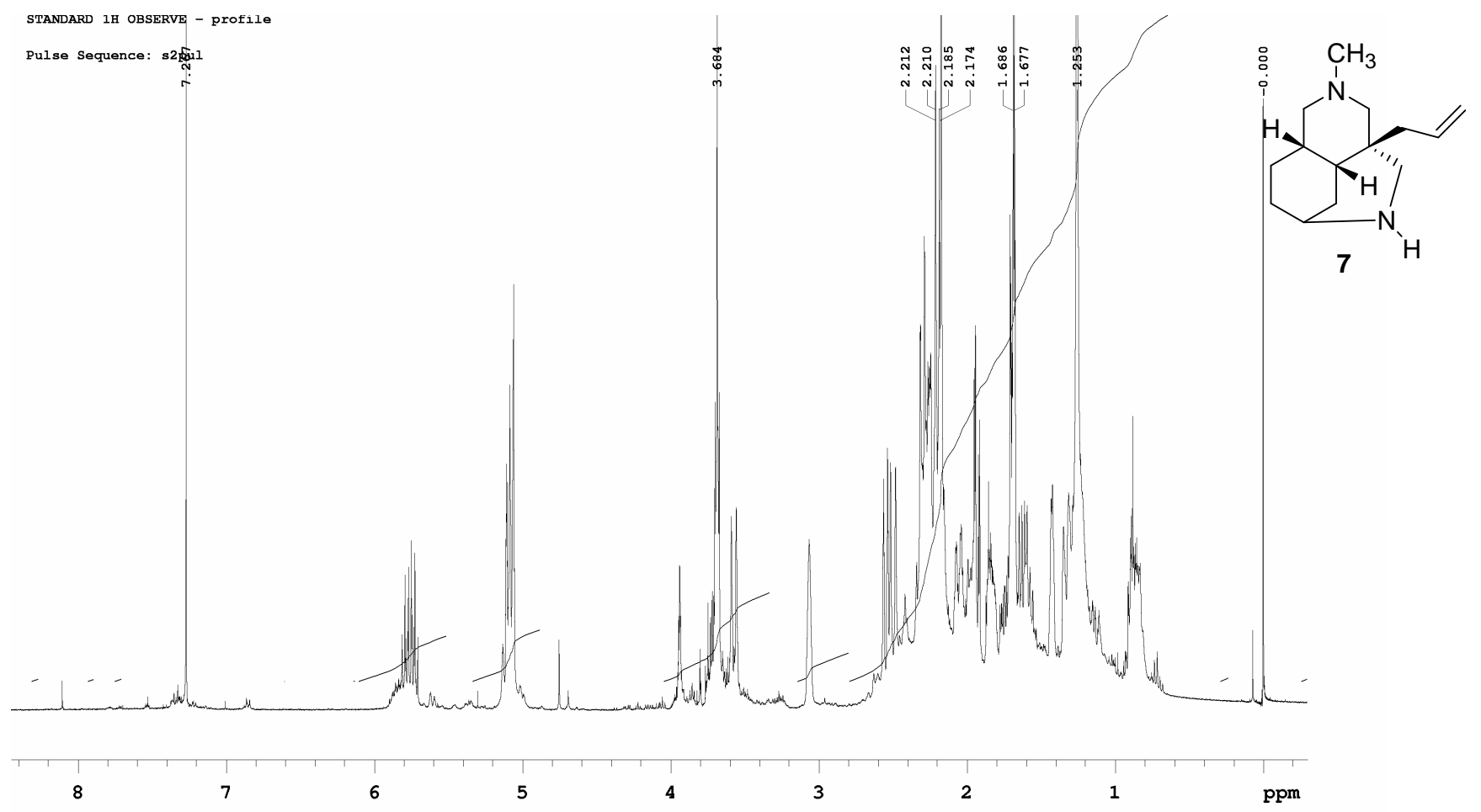

vnmrs 400

cdc13/temp. 25C/N. reg: 2207-2006

usuar1:san/mostra: 1pf2 juntes

Pulse Sequence: s2pul
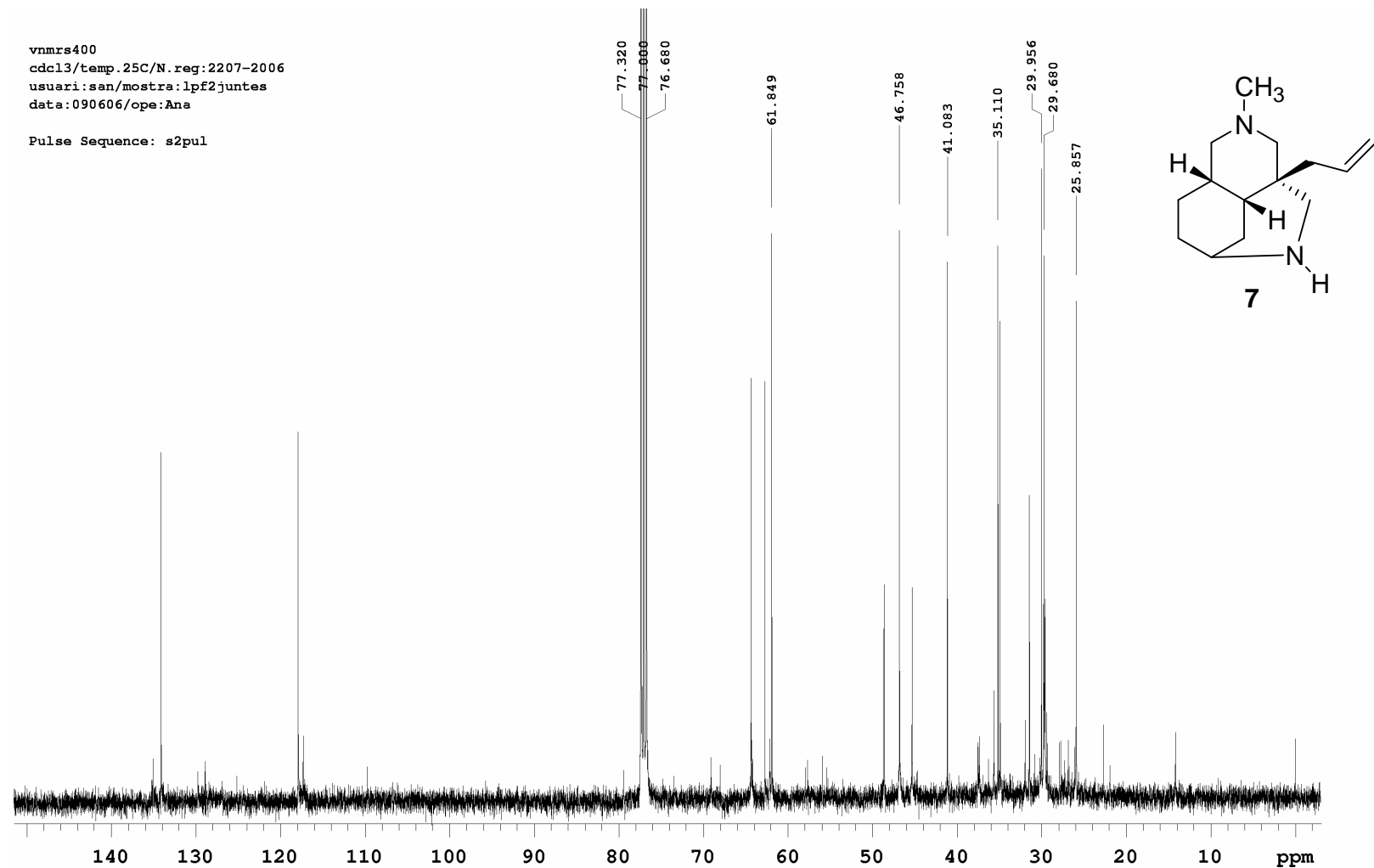
vnmrs 400

. 25C/N.reg:2207-2006 usuri:san/mostra:lpf2juntes

data : 090606/ope: Ana

Pulse Sequence: gcosY

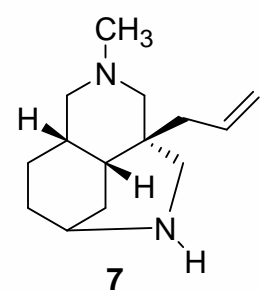

7

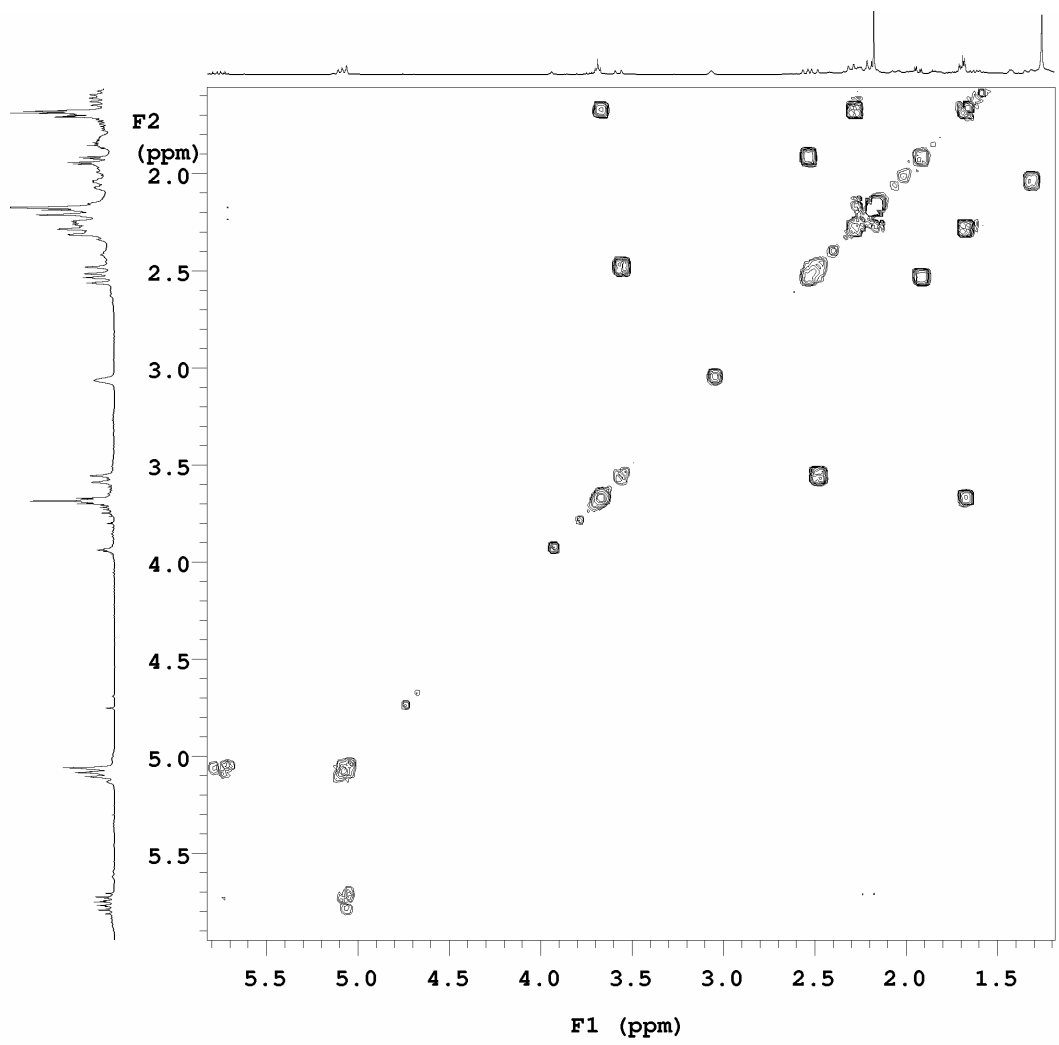

vnmrs 400

cdc13/temp. 25C/N. reg: 22 07-2006

usuari: san/mostra : 1pf2 juntes

data : 090606/ope: Ana<smiles>C=CC[C@H]1CNC2CC[C@H]1CN2</smiles>

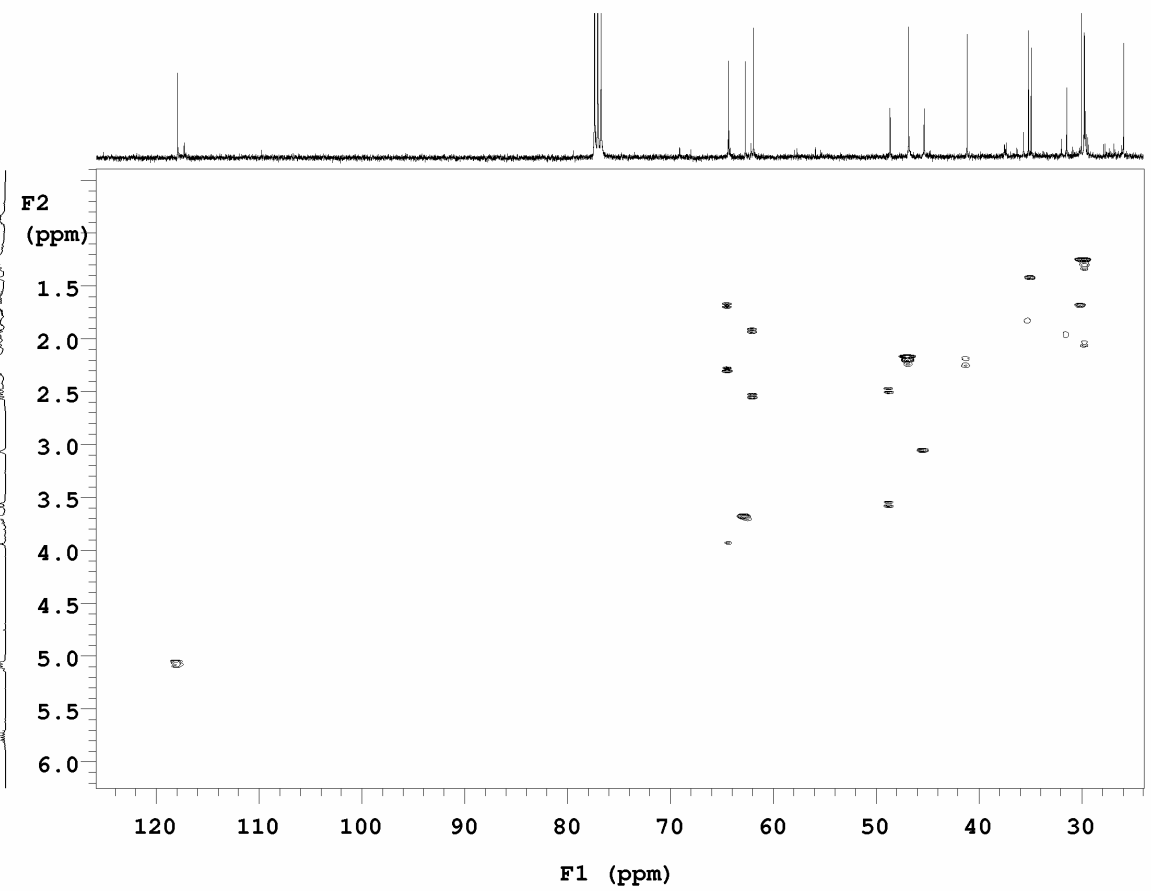


H1 / s2pul / Mercury-400F

Usuari:

Data:27/06/06/Op.:Ana

Pulse Sequence: s2pul

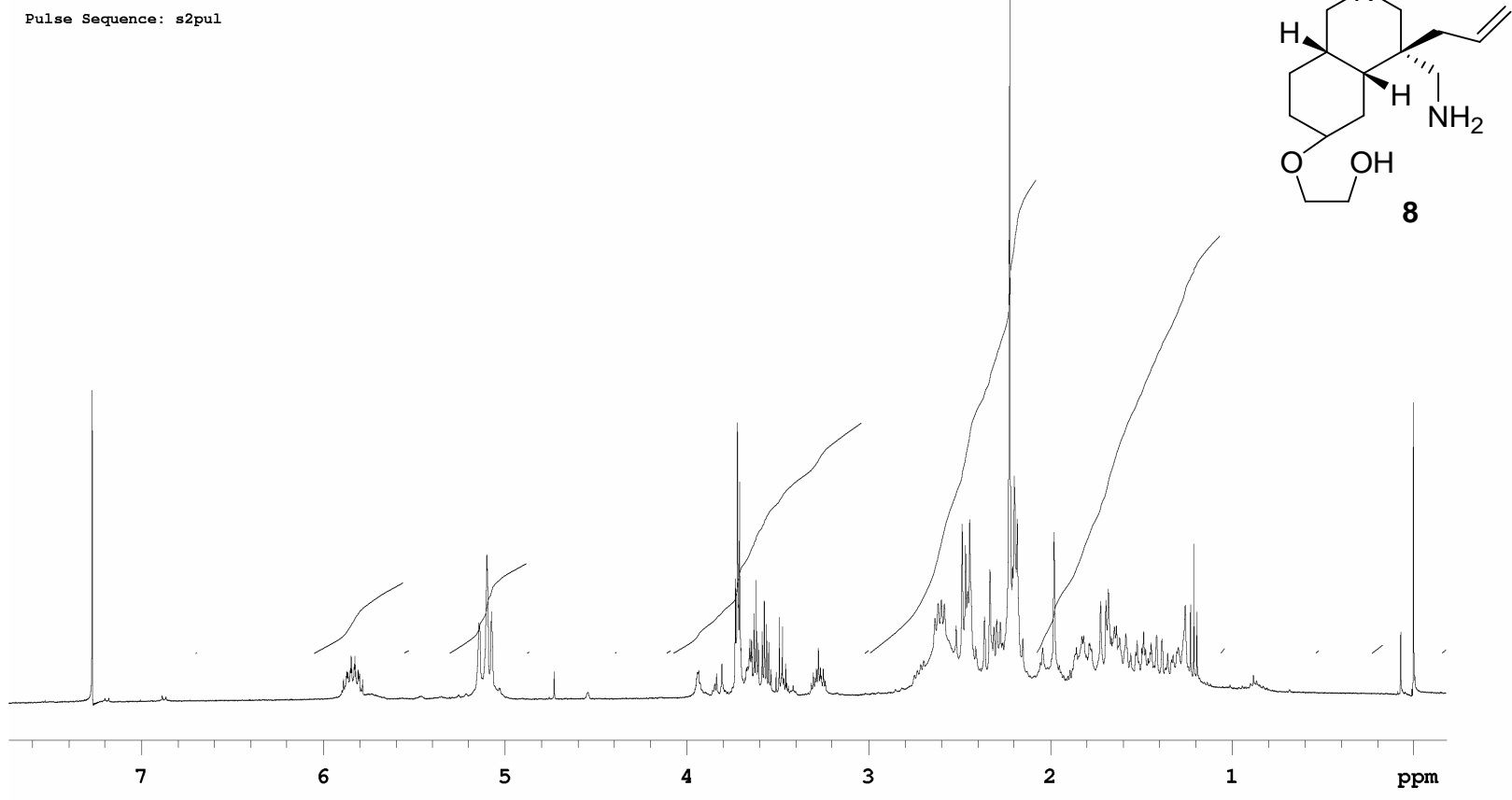

vnmrs 400
vnmrs 400

cdc13/ temp. 25C/ N reg: 2034-2006

usuari: san/mostra: 1palc13f3

오ำ

data: 30-05-06/ ope: Ana

i

Pulse Sequence: s2pul

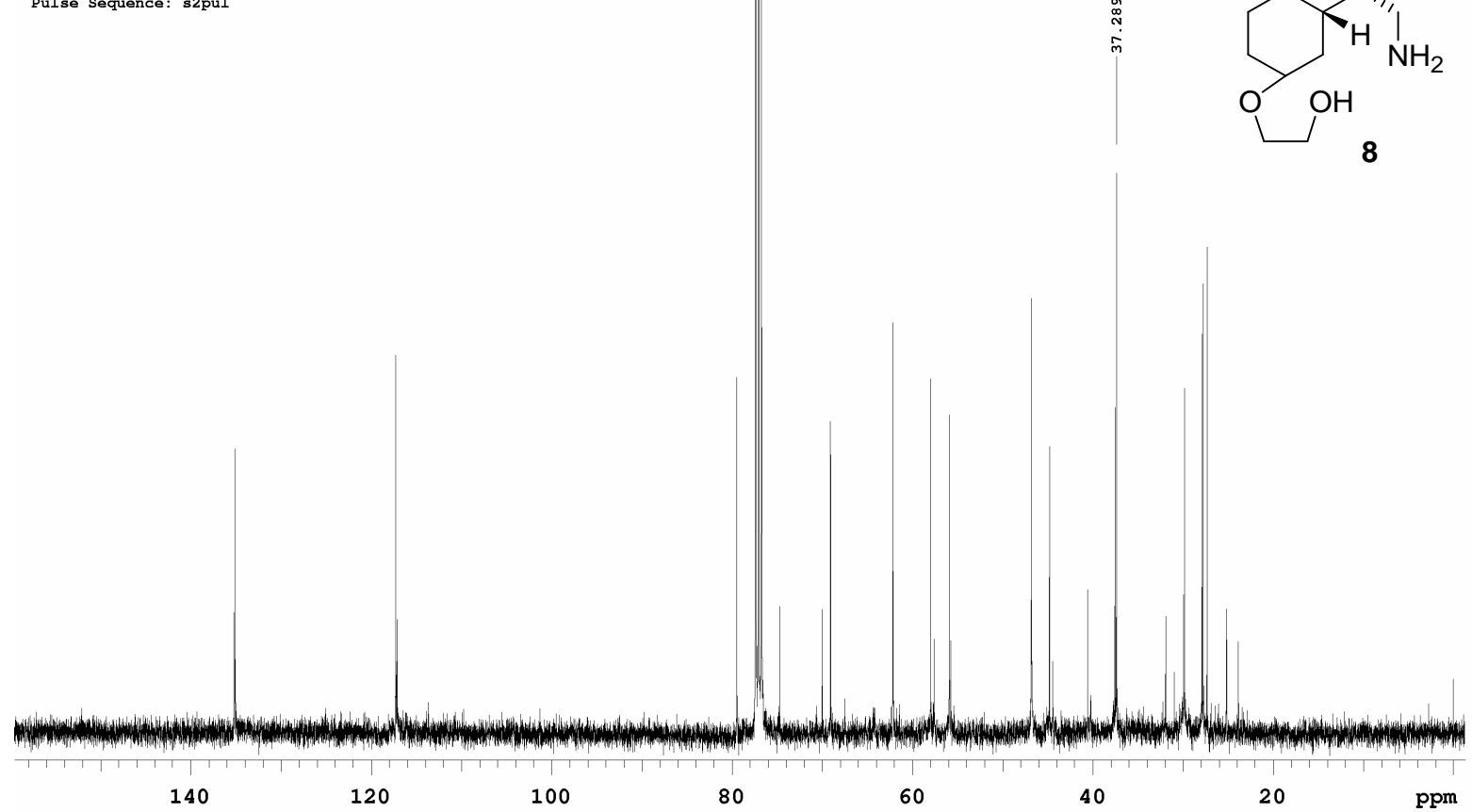


H1 / gcosy/ Mercury-400F

Usuari: san / Mostra: lpalc1 6 / Op. : Ana

Pulse Sequence: $\cos \mathrm{CY}$<smiles>C=CC[C@]1(CN)CN(C)C[C@H]2CCC3CC2C31</smiles>

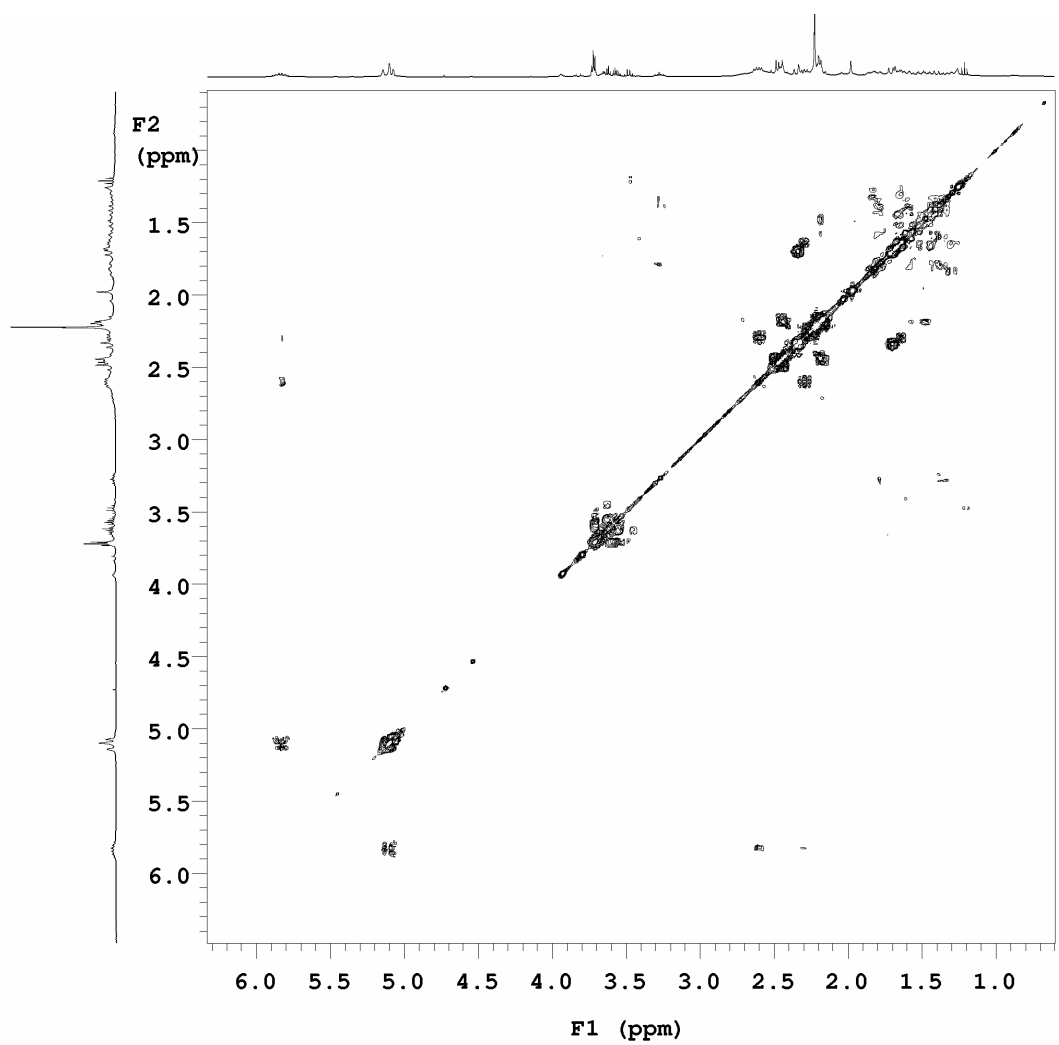

H1 / gHSQC / Mercury-400F

Cdc13/ 25C/N reg: 02477-2006

Usuar1: san / Mostra: 1 pa

Pulse Sequence: gHSQC<smiles>C=CC[C@]1(CN)CN(C)C[C@H]2CCC3OCCOCC3[C@H]21</smiles>

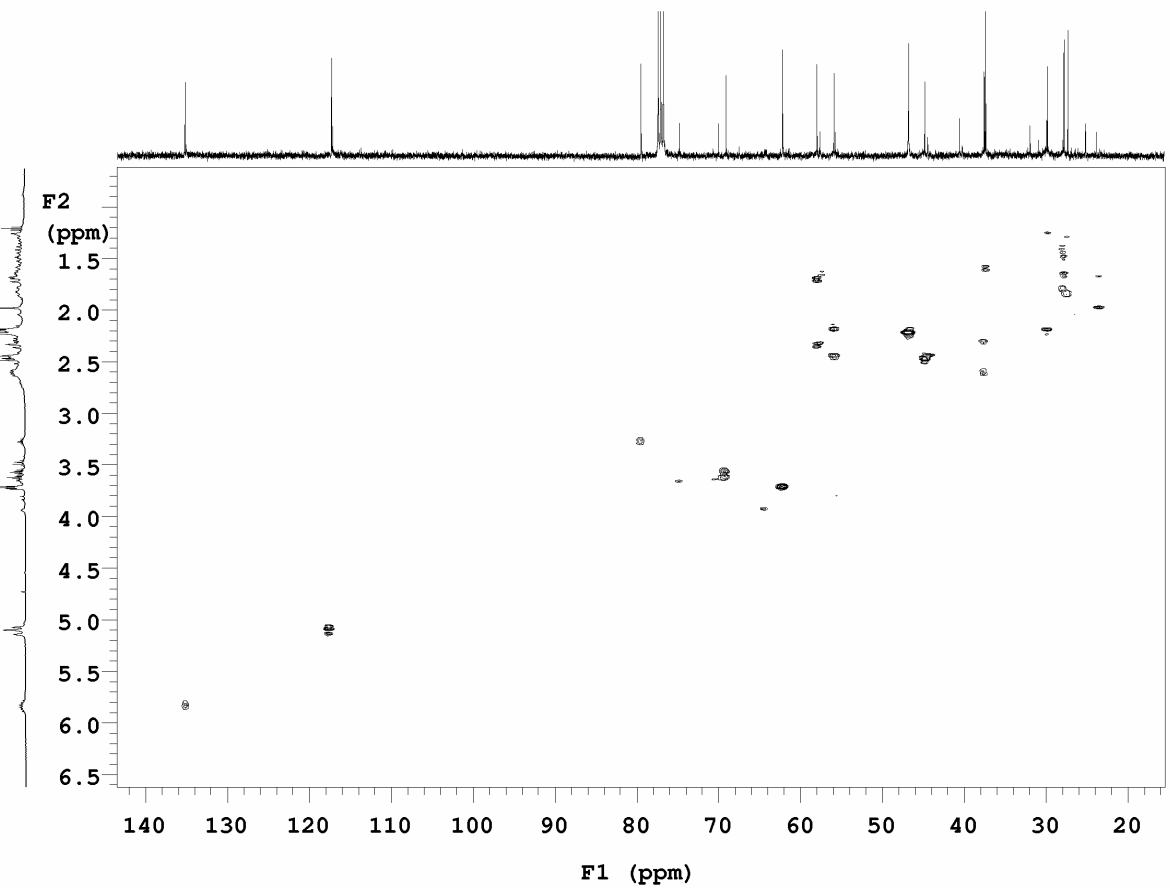


H1 / s2pul / Mercury-400_qui

cdc13/Temp: 25C /N reg: M40007-01080716253

Usuari: san / Mostra: 1p14

Deta: $01 / 00 / 07 /$ sist

Pulse Sequence: s2pul

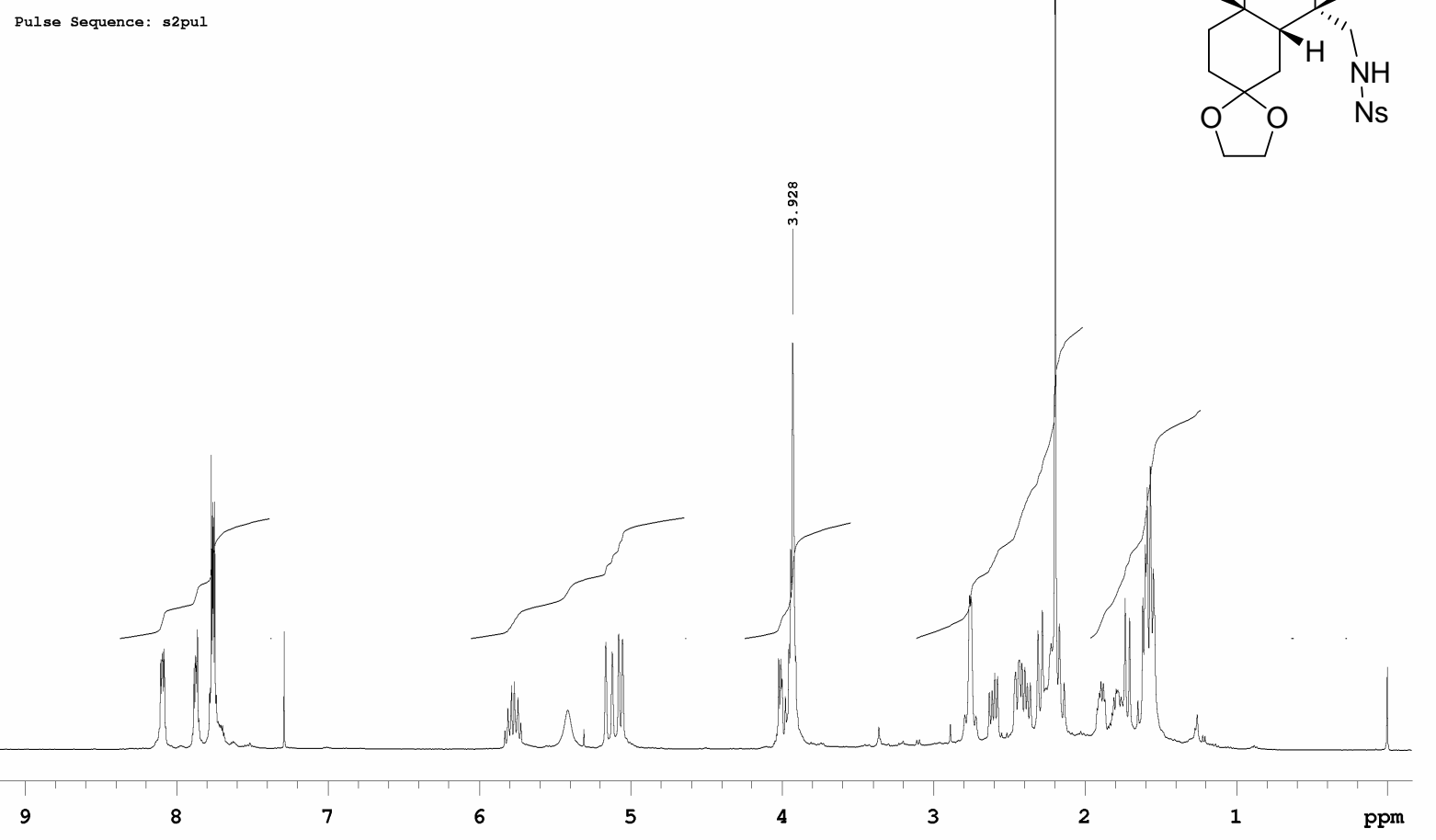

C13 / s2pul / Mercury-400_qui

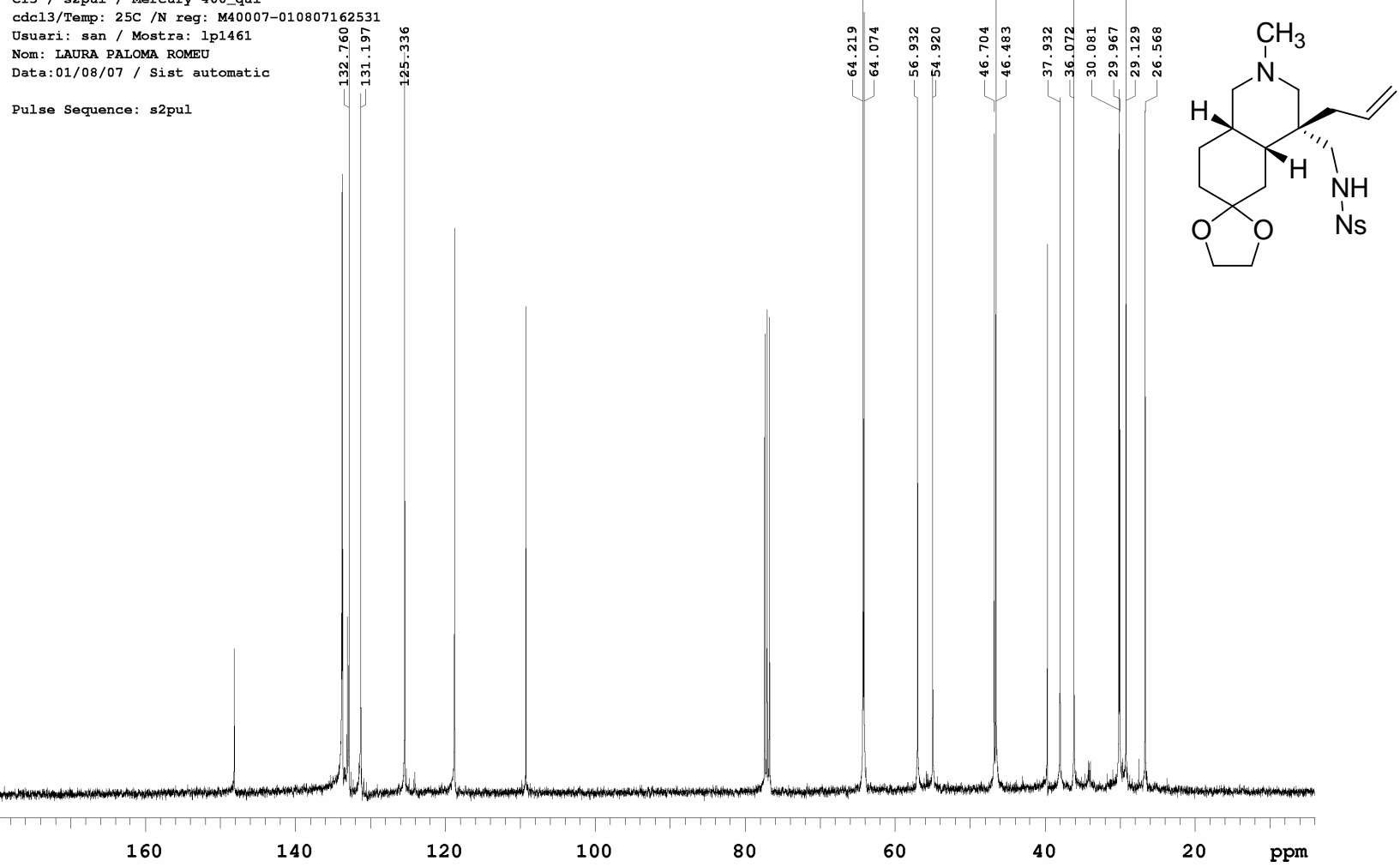


H1 / gCosy / Mercury-400_qui

cdc13/Temp: 25C /N reg: M40007-01080716253

Usuari: san / Mostra: 1p1461

Data: 01/08/07/ Sist automatic

Pulse Sequence: gcosY
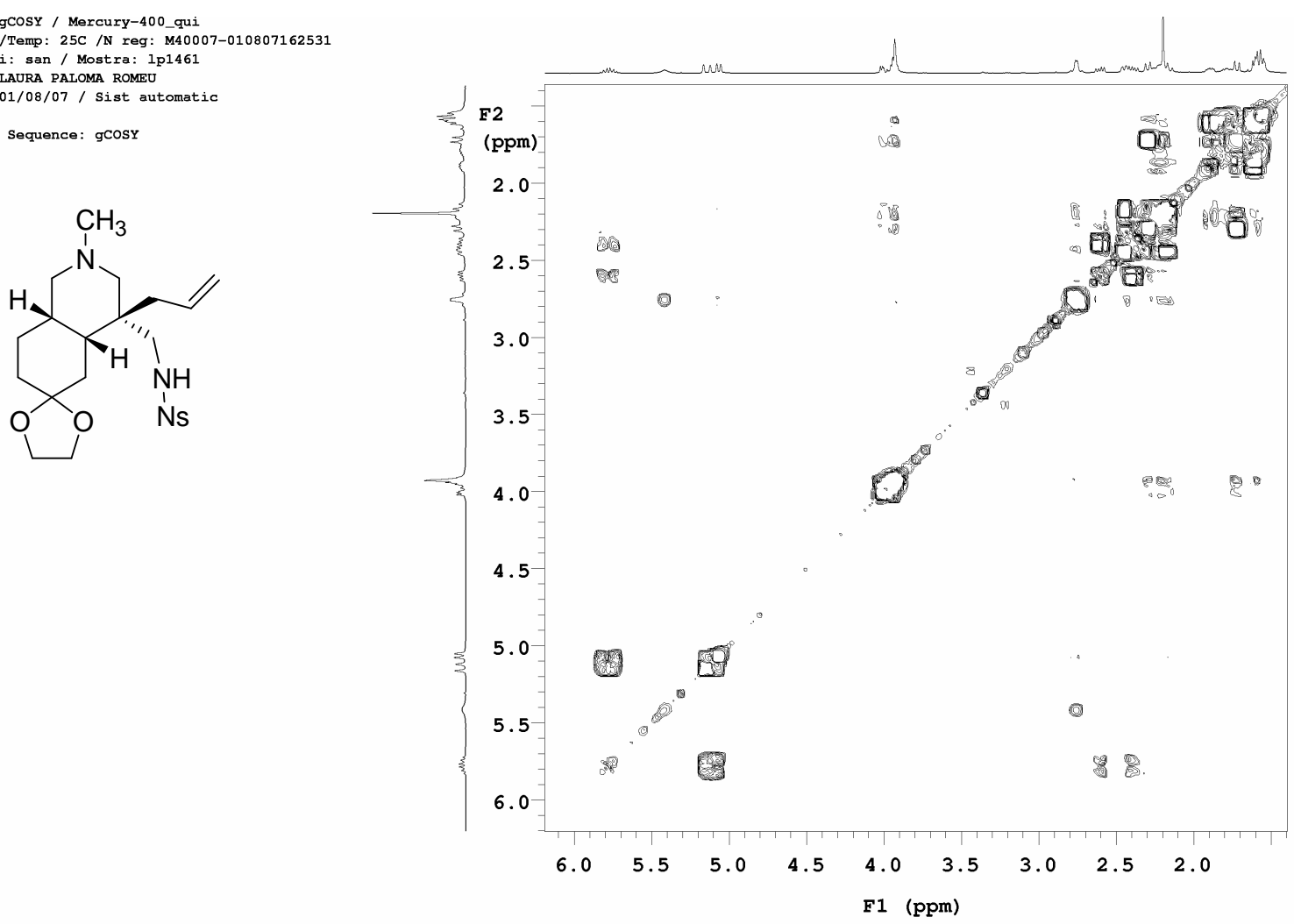

H1 / gHSQC / Mercury-400_qui
cdc13/Temp: 25C /N reg: M40007-010807162531

Usuari: san / Mostra: 1p1461

Pulse Sequence: gHSQC<smiles>C=CC[C@]1(CNN)CN(C)C[C@H]2CCC3(C[C@H]21)OCCO3</smiles>

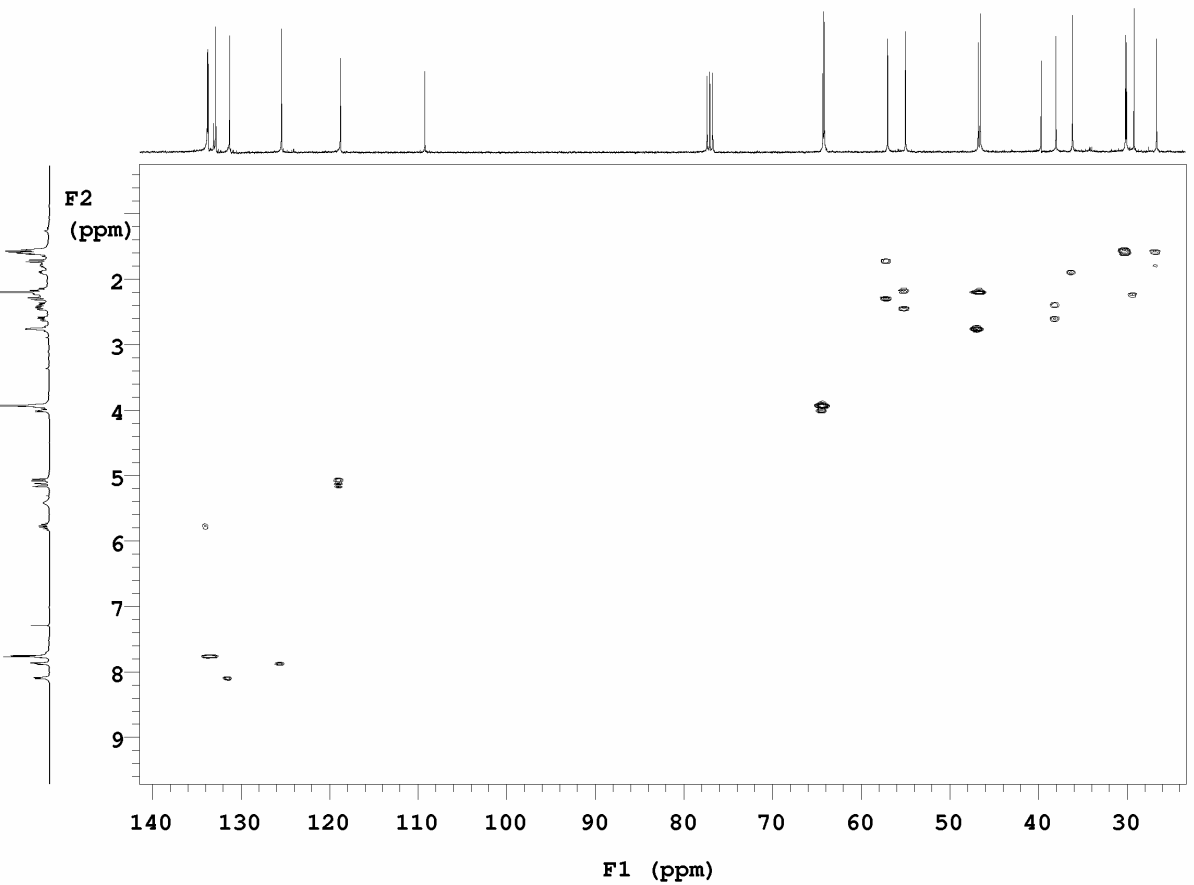


H1 / s2pul / Mercury-400_qui

6-131006180121

Nom: LAURA PALOMa ROMEU

Data: $13 / 10 / 06$ / Sist automatic

Pulse Sequence: s2pul

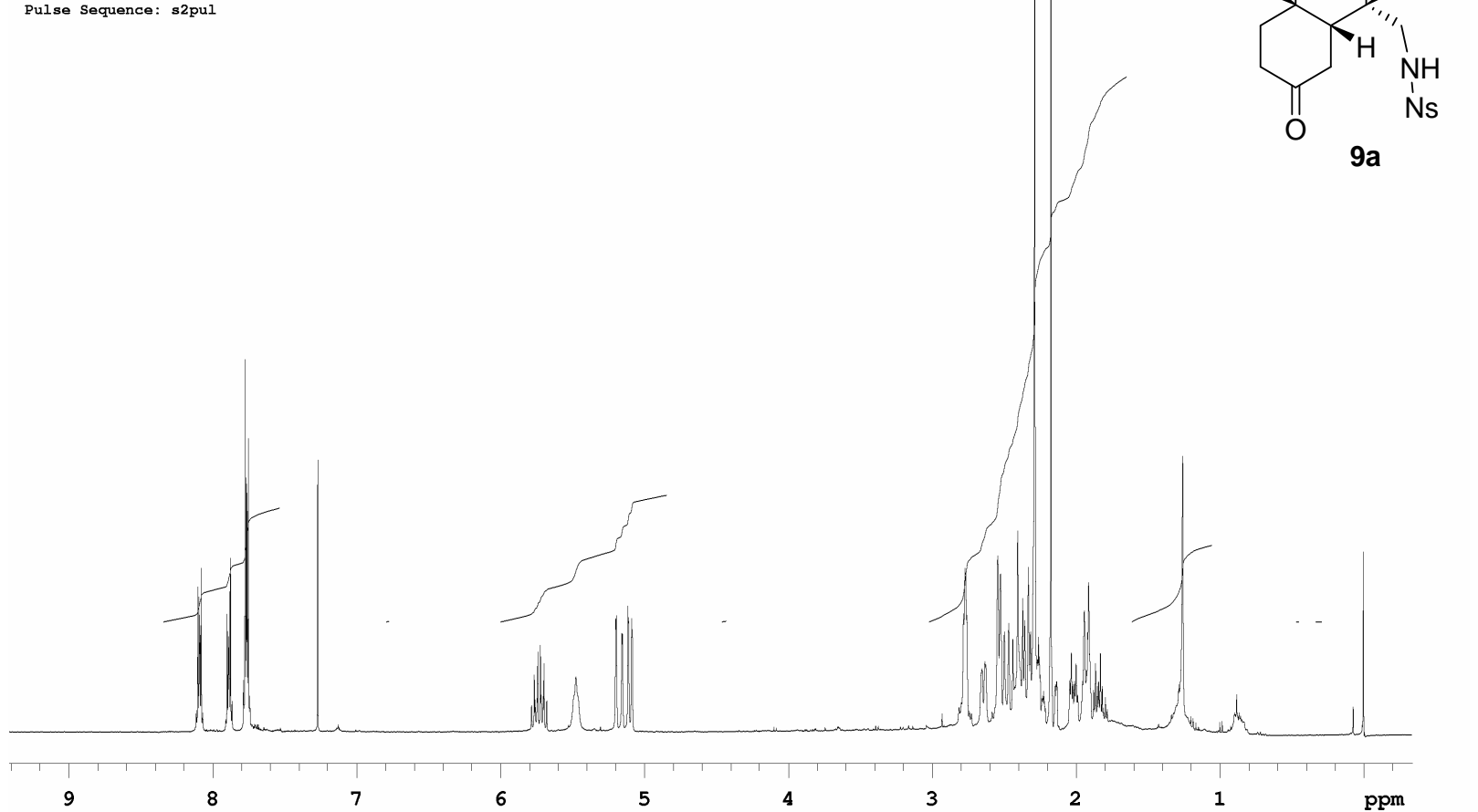

C13 / s2pul / Mercury-400_qui

cdc13/Temp: 25C /N reg: M40006-13100618012

Usuari: san / Mostra: Ip1315

WOM: LAURA PALOMA ROMEU

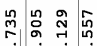

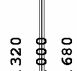

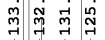

F

Pulse Sequence: s2pul

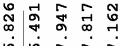

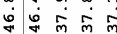

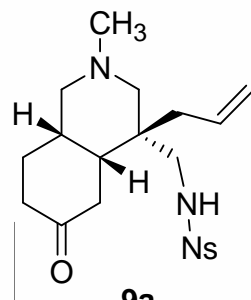

9a

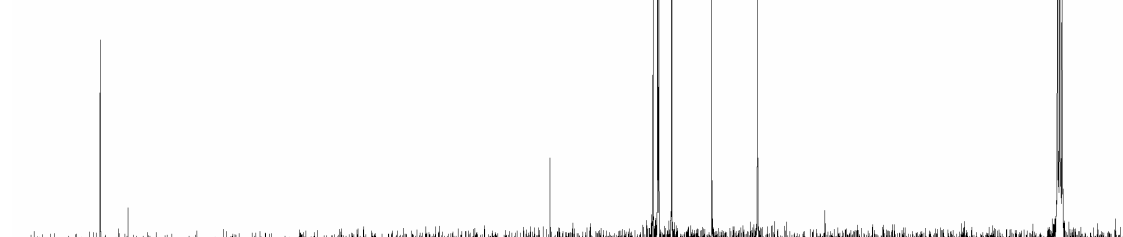

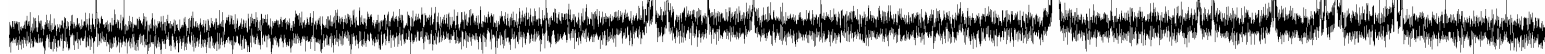


H1 / gCOSY / Mercury-400_qui

cdc13/Temp: 25C /N reg: M40006-131006180121 Usuari: san / Mostra: 1p1315 Nom: LAURA PALOMA ROME

Data:13/10/06 / Sist automatic

Pulse Sequence: gcosy<smiles>C=CC[C@]1(CNN)CN(C)C[C@H]2CCC(=O)C[C@H]21</smiles>

9a

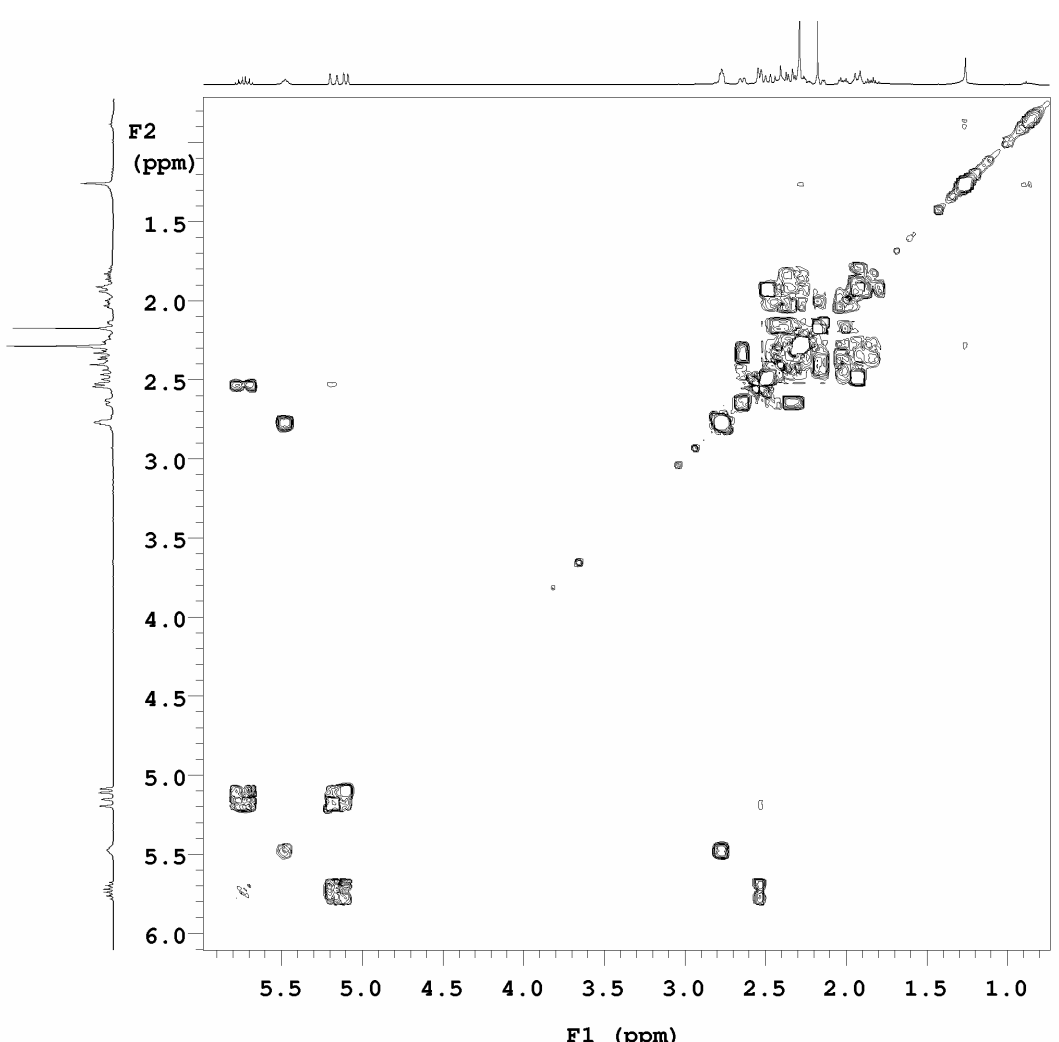

F1 (ppm)

11 / gHSQC / Mercury-4000 quB

cdc13/Temp: 25C /N reg: M40006-131006180121

Usuari: $s$ an / Mostra: 2pi315

Om: LAURA PALOMA ROMEU

Data:13/10/06 / Sist automatic

Pulse Sequence: gHSQC<smiles>C=CC[C@]1(CNN)CN(C)C[C@H]2CCC(=O)C[C@]21C</smiles>

$9 a$

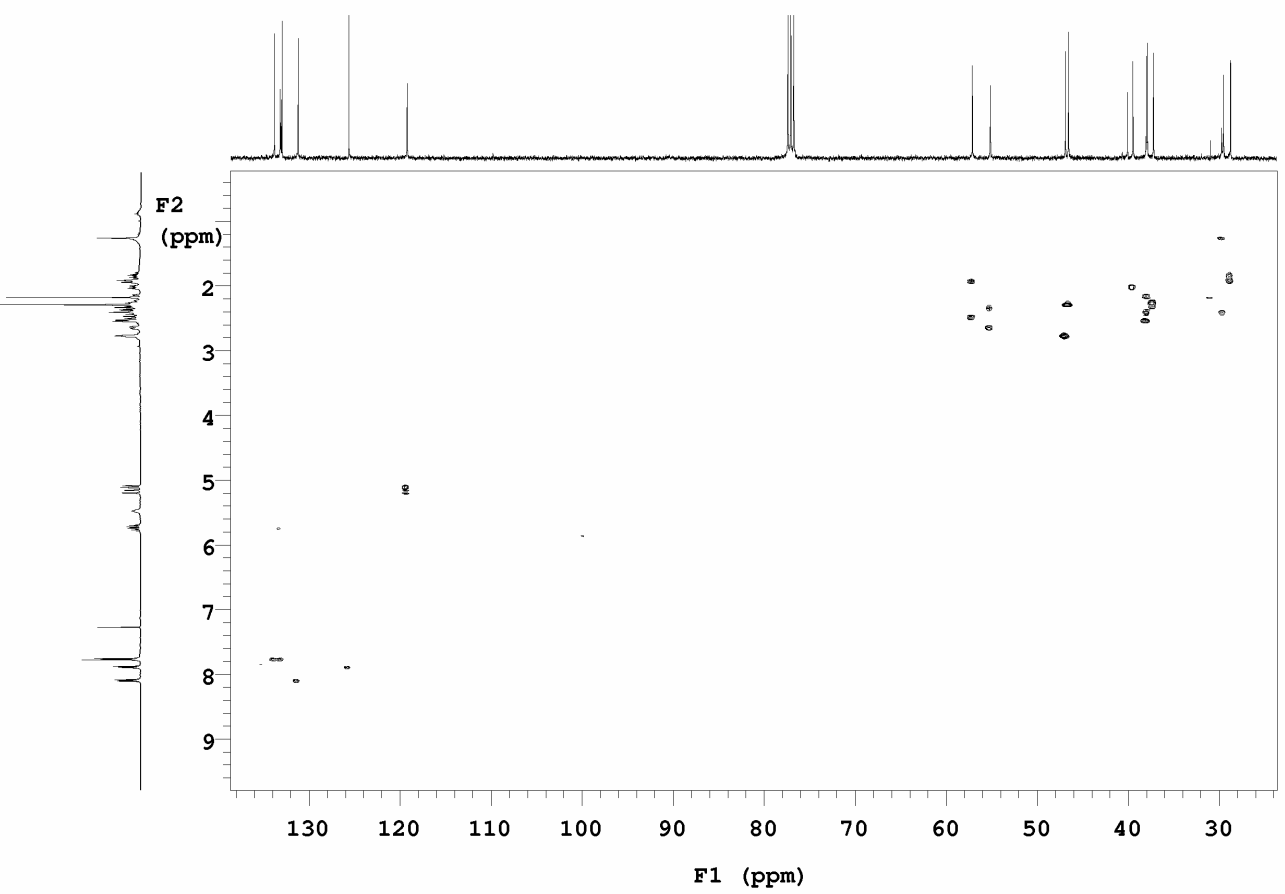


H1 / s2pul / Mercury-400_qui

Usuari: san / Mostra: 1p1397

RA PALOMA ROMEU

Data:23/11/06 / Sist automatic

Pulse Sequence: s2pul

†

(n)

.

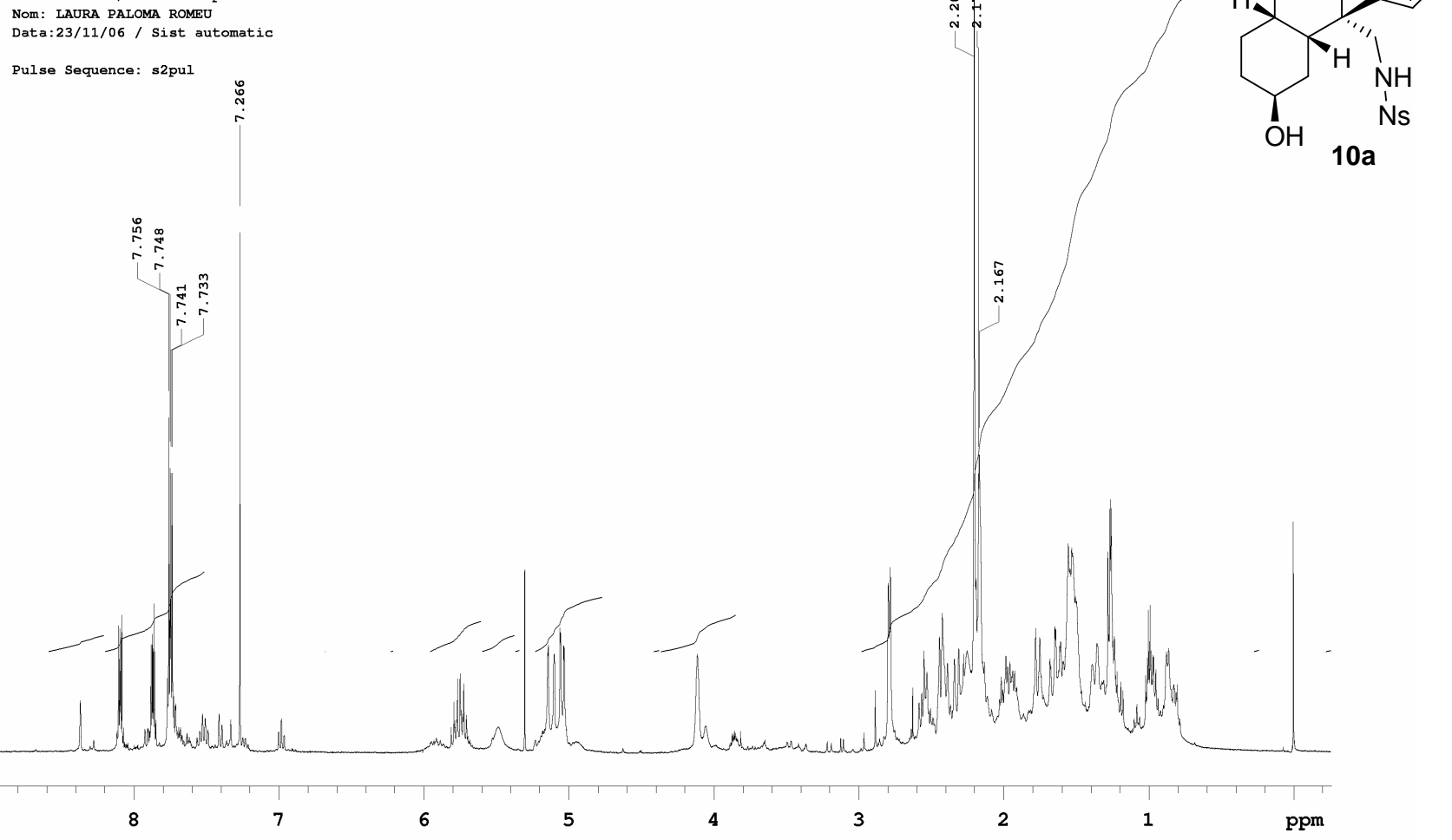

C13 / s2pul / Mercury-400_qui

cdc13/Temp: 25C/N reg: M40006-221106181018

Usuari. san / Mostra: 1p1397

Data:23/11/06 / Sist automatic

Pulse Sequence $\underset{\substack{\infty \\ \sim}}{\min } \mathbf{s}$ pul

m
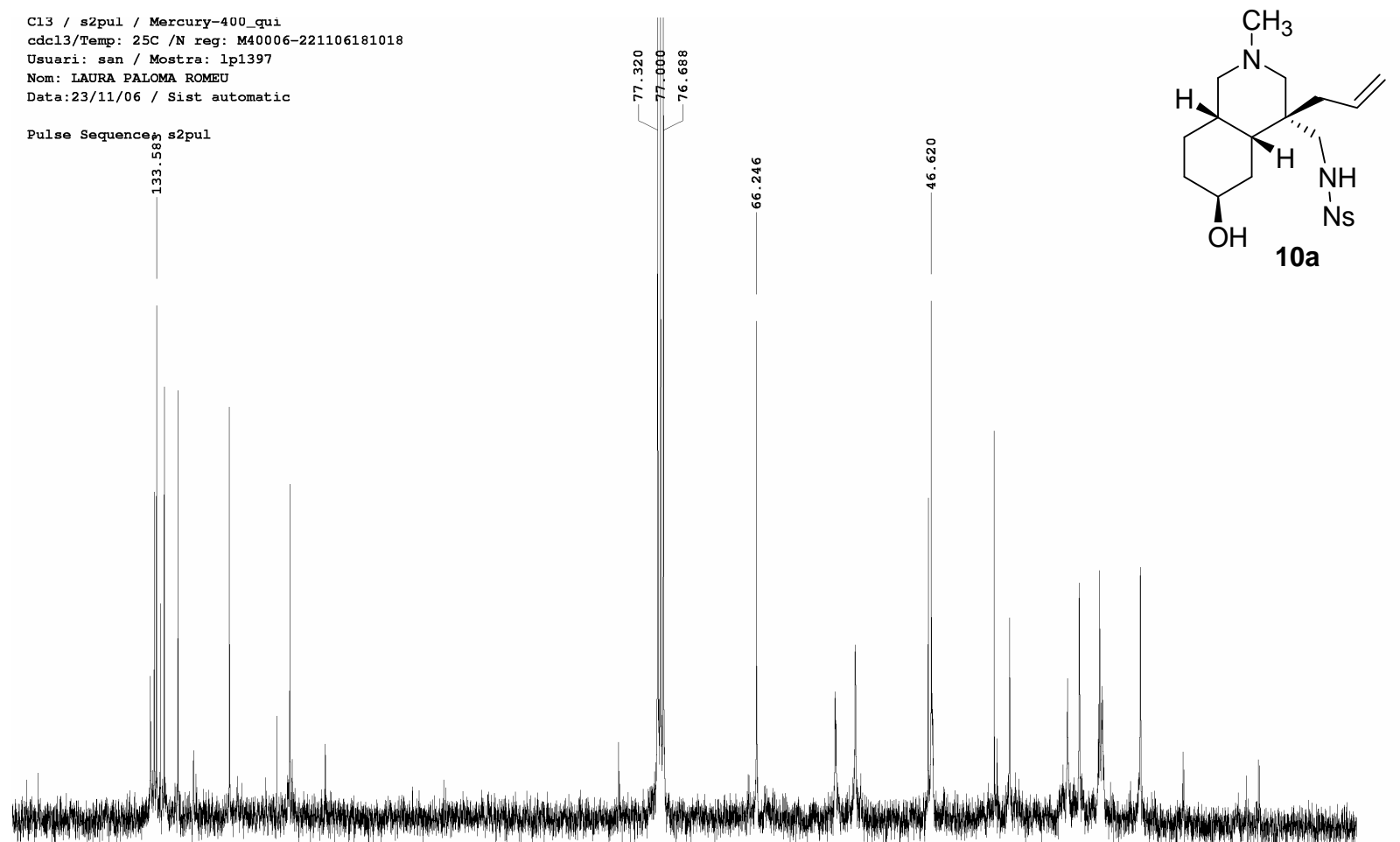

$\because$

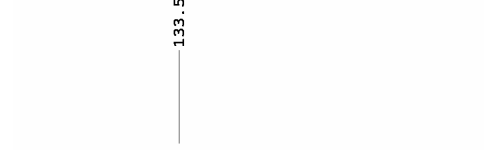


H1 / gCosy / Mercury-400_qui

M40006-221106181018

Us:

Data:23/11/06/ Sist automatic

Pulse Sequence: gcosy
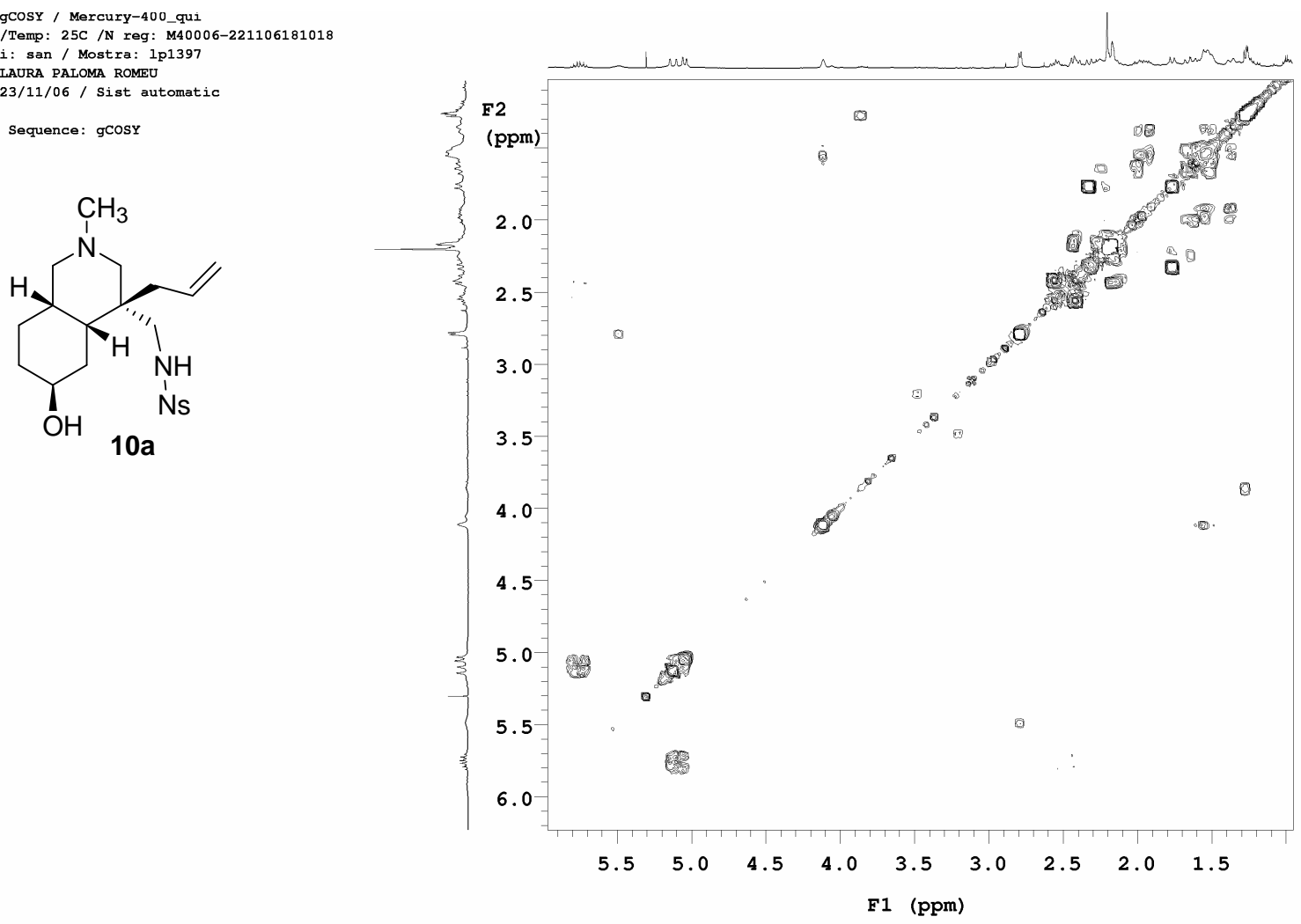

H1 / gHSQC / Mercury-400_qui

cdc13/Temp: 25C /N reg: M40006-22110618101

Usuari: san / Mostra: 1p1397

Data:23/11/06 / Sist automatic

Pulse Sequence: gHSQC<smiles>C=CC[C@]1(CNN)CN(C)C[C@H]2CCC(O)C[C@H]21</smiles>

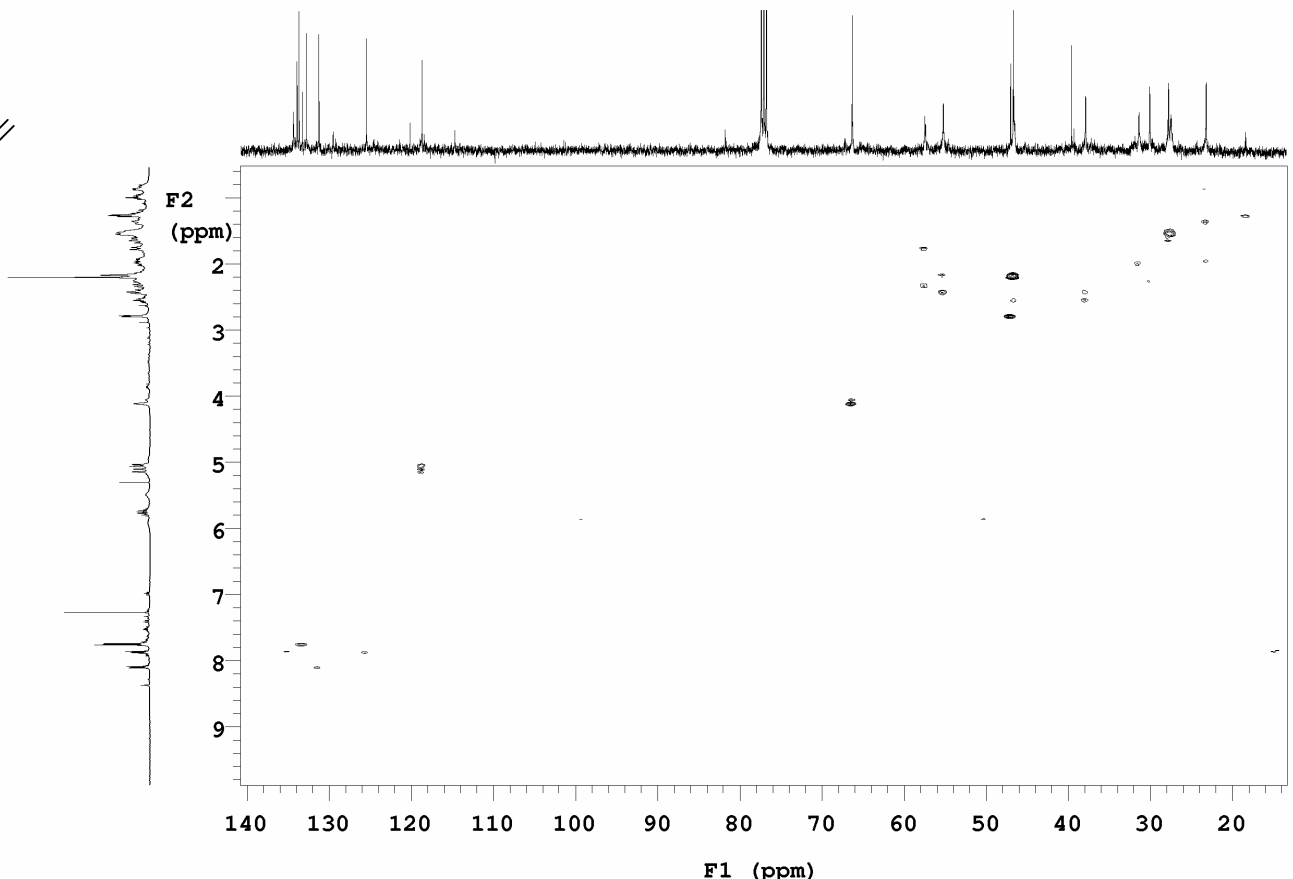


H1 / s2pul / Mercury-400F
cdc13 / Temp: 25C / N.Reg: 3383/2006

Usuari: san / Mostra: 1p1293

Nom: LAURA PALOMA ROMEU
Data: 06/10/06/ Ope.: A.LINARES

Pulse Sequence: s2pul

每

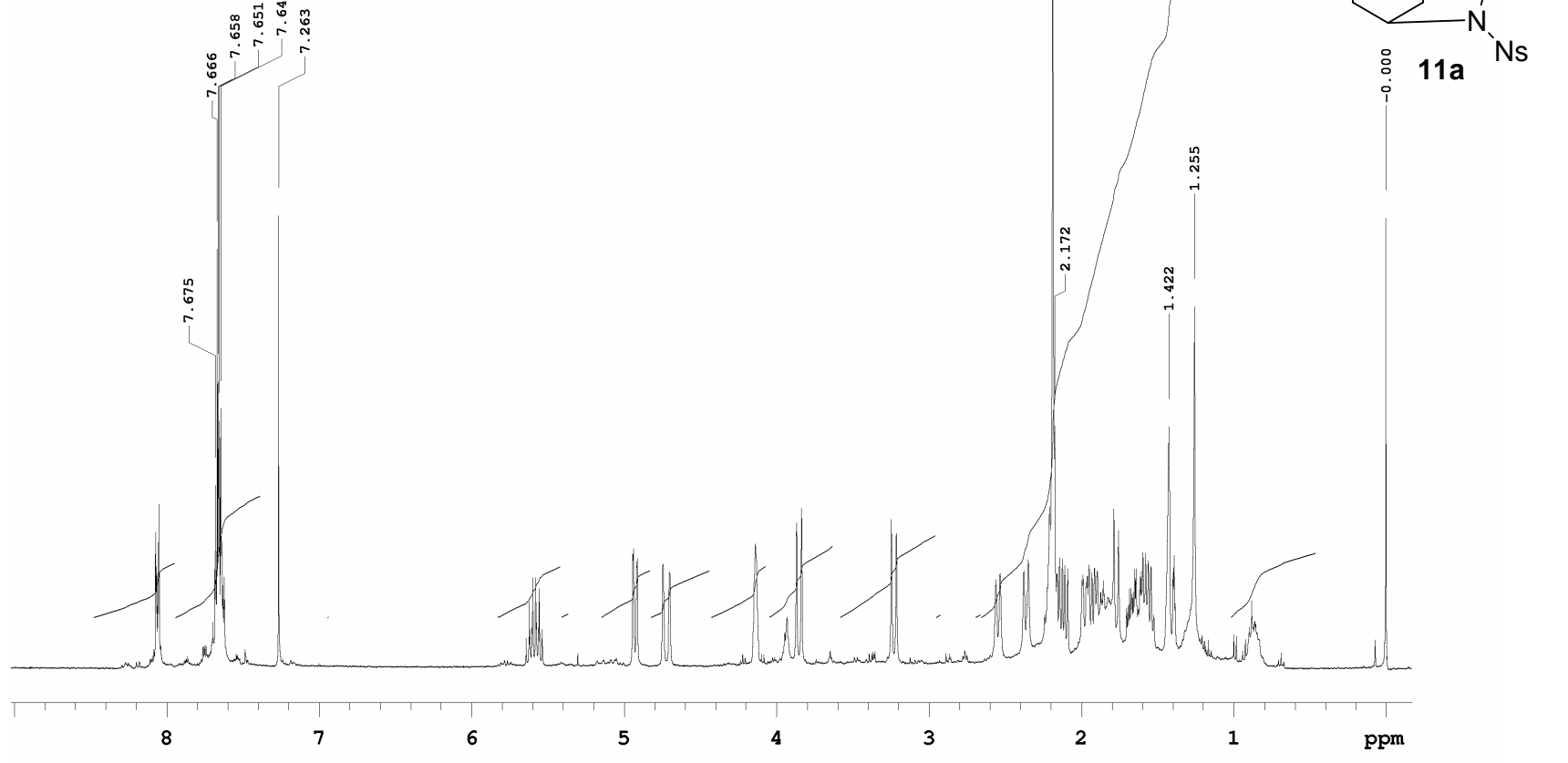

C13 / s2pul / Mercury-400F

cdc13 / Temp: 25C / N.Reg: 3383/2006

Usuari: san / Mostra: 1p1293

Data: 06/10/06/Ope.: A.I INNABEE

Pulse Sequence: s2pul

둥 윰

F

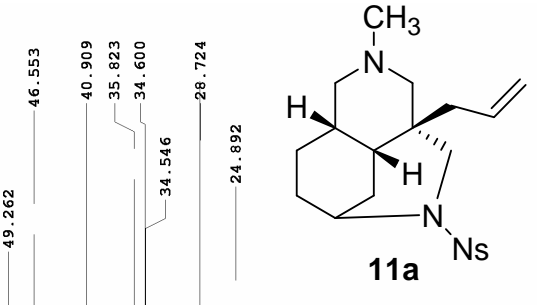

160

140

120

100

80

60

40

20

ppm 
H1 / gcosy / Mercury-400F

eg: $3383 / 2006$

1p1293 / LAURA

Data: 06/10/06/Ope : Ana

Pulse Sequence: gcosY

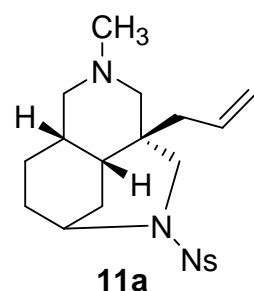

$11 \mathrm{~N}$ Ns

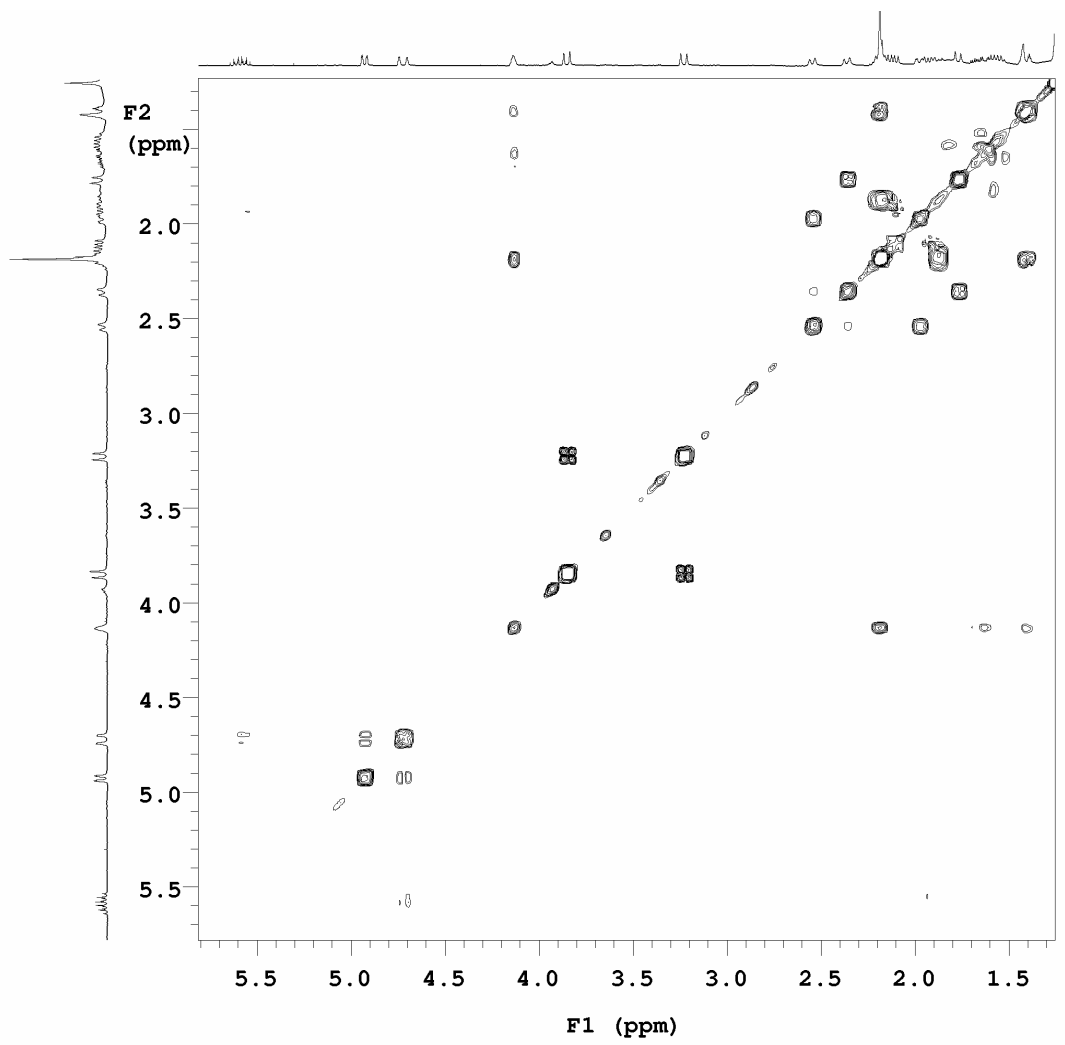

H1 / ghsqc/ Mercury-400F

cdc13 / Temp: 25C / N.Reg: 3383/2006

Usuari: san / Mostra: 1p1293 / LAURA

Data: 06/10/06/Ope.: Ana

Pulse Sequence: gHSQC<smiles>C=CC[C@]1(CN(C)C)CN(C)CC2CCCCC21</smiles>
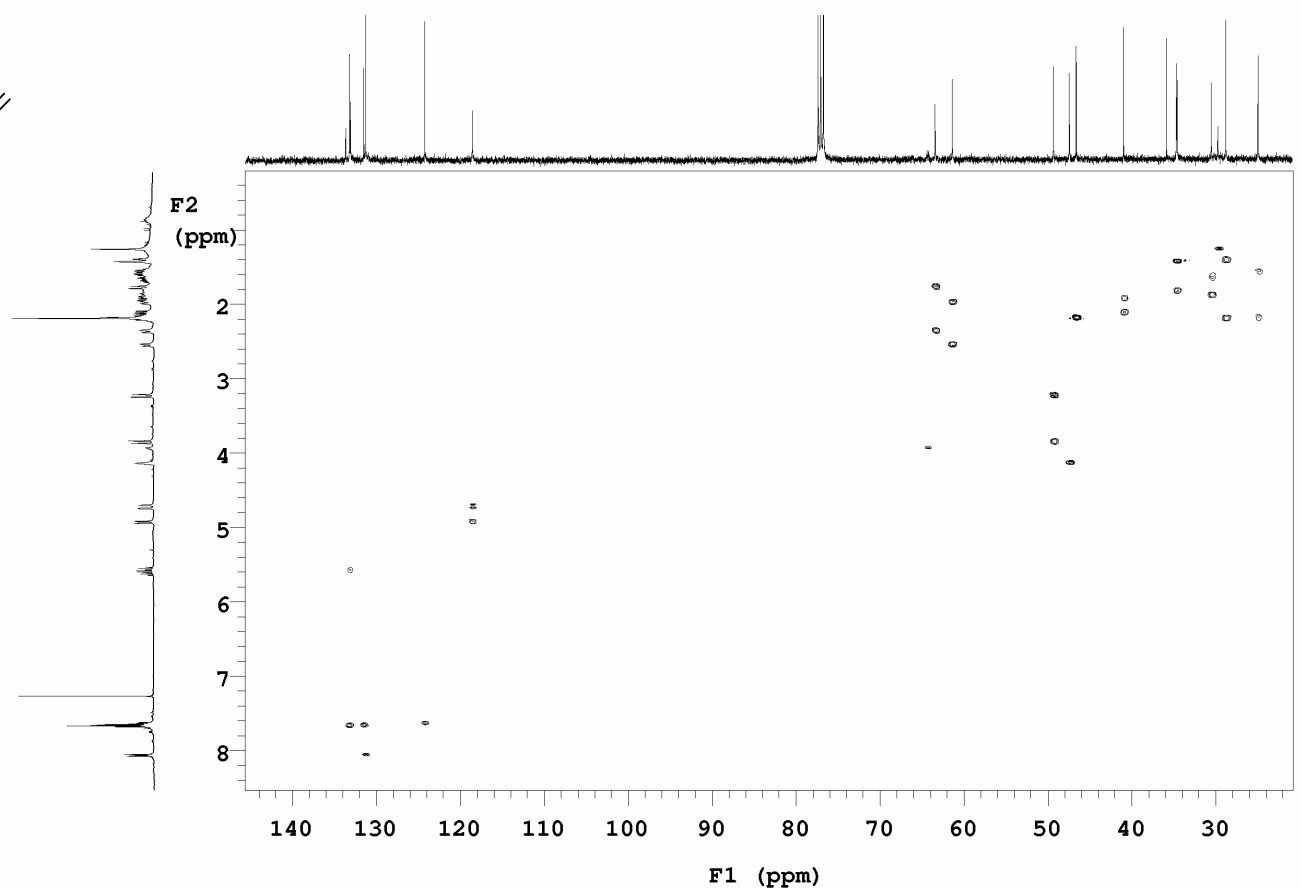
H1 / s2pul / Mercury-400F

cdc13 / Temp: 25C / N.Reg: $x x x x x x x x x x$

Usuari: san / Mostra: 1p1597

NOM: XAVIER VILA TUSELL

Data: 28/03/07 / Ope.: X.VILA

Pulse Sequence: s2pul
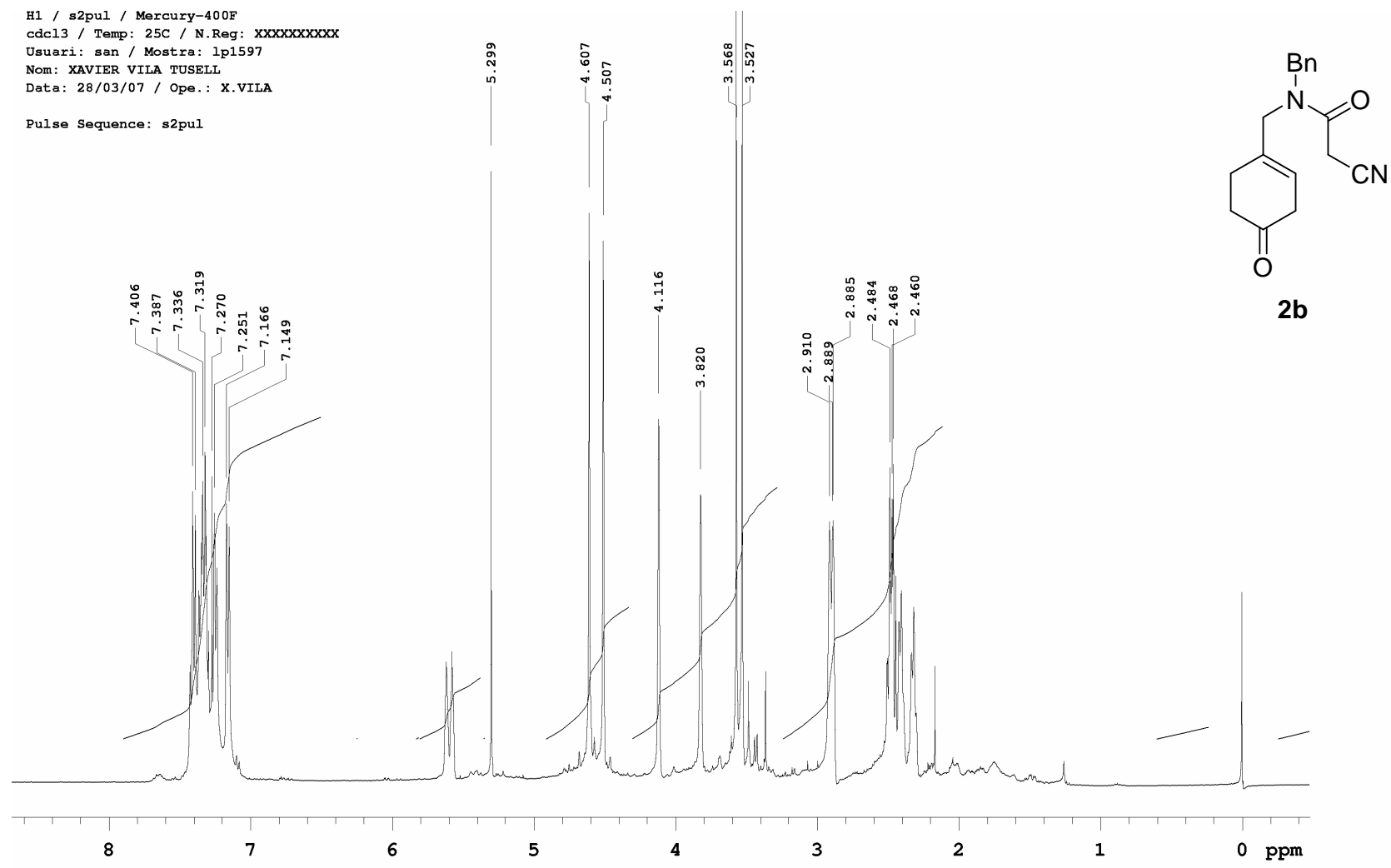

C13 / s2pul / Mercury-400F

dc13/Temp: $25 C /$ N. Reg: $x x x x x x x x x x$

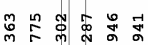

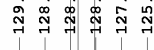

Pulse Sequence: s2pu-

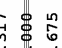

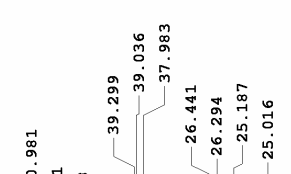<smiles>N#CCC(=O)N(CC1=CCC(=O)CC1)Cc1ccccc1</smiles>

$2 b$

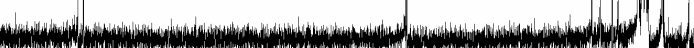


H1 / gCOSY / Mercury-400F

cdc13 / Temp: 25C / N.Reg: $x x \times x \times x x x x x$ Usuari: san / Mostra: 1p1597 NOm: XAVIER VILA TUSELI

Data: 29/03/07 / Ope.: X.vILA

Pulse Sequence: gCosY<smiles>N#CCC(=O)N(CC1=CCC(=O)CC1)Cc1ccccc1</smiles>

$2 b$

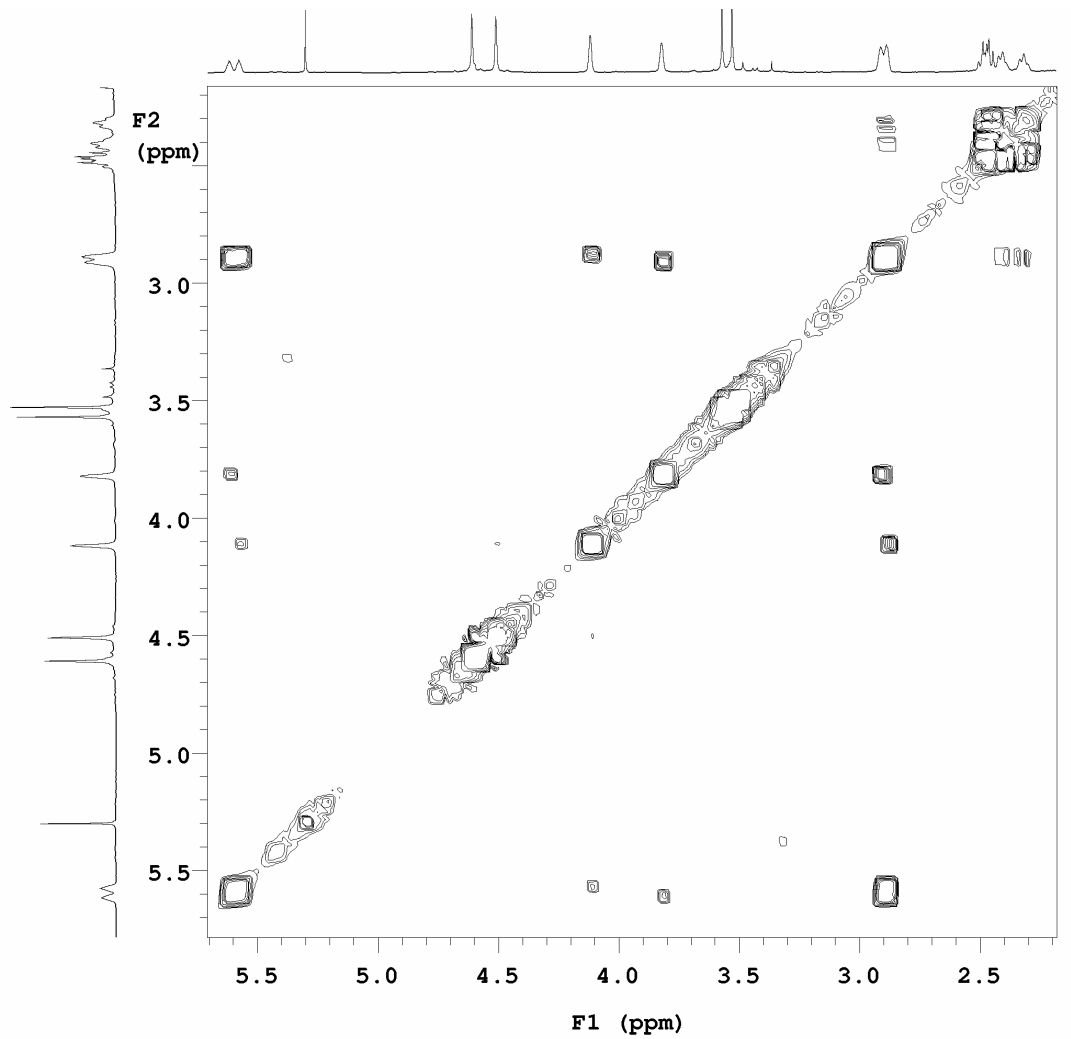

H1 / gHSQC / Mercury-400F

cdc13 / Temp: 25C / N.Reg: xxxxxxxxxx

Jsuari: san / Mostra: 1p1597

Data: $29 / 03 / 07 /$ TUSELL

Pulse Sequence: gHSOC<smiles>N#CCC(=O)N(CC1=CCC(=O)CC1)Cc1ccccc1</smiles>

$2 b$

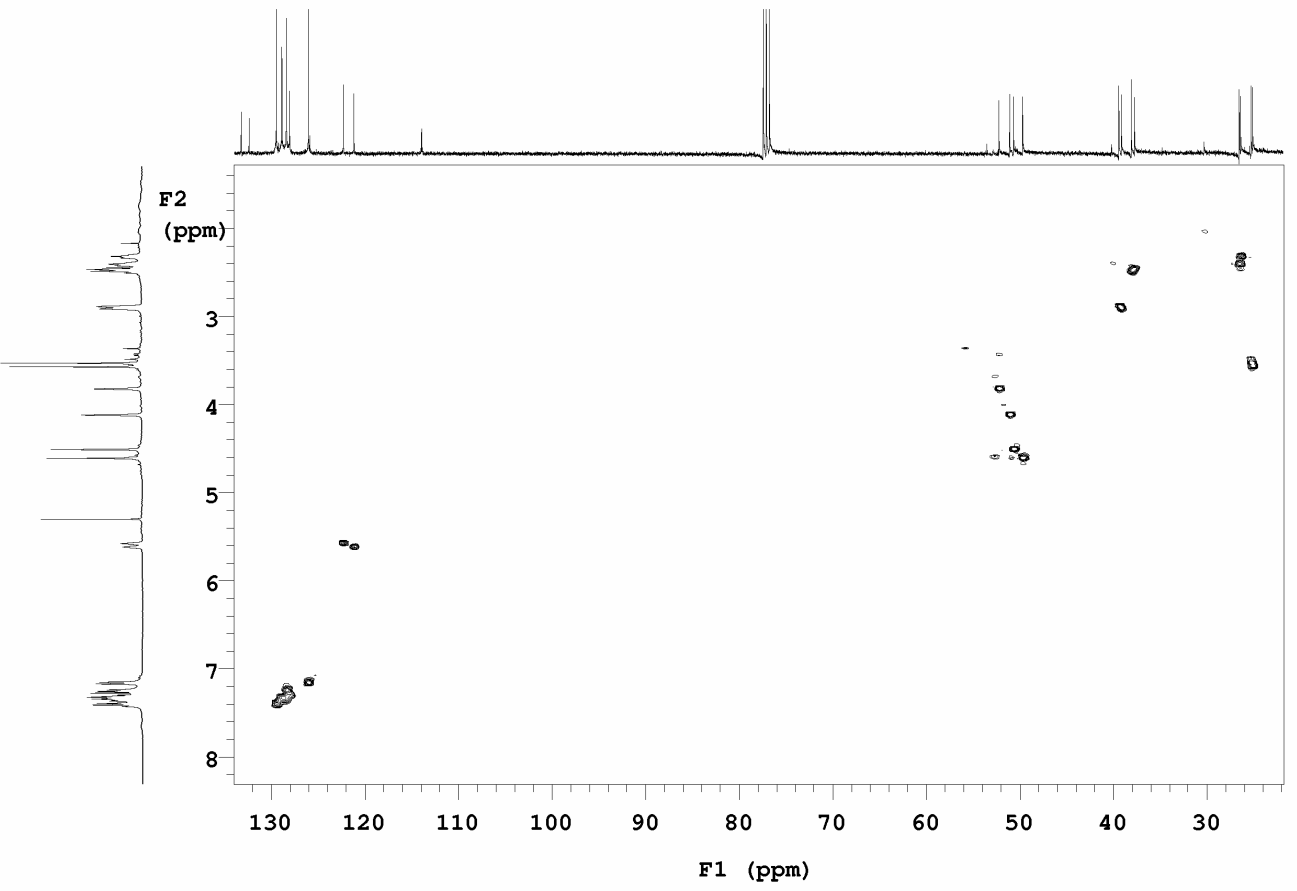


H1 / s2pul / Mercury-400_qui

cdc13/Temp: 25C /N reg: M40004-221004164958

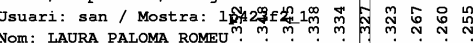

Data:23/10/04/ Sist aufomatic

Pulse Sequence: s2pul

ijitio

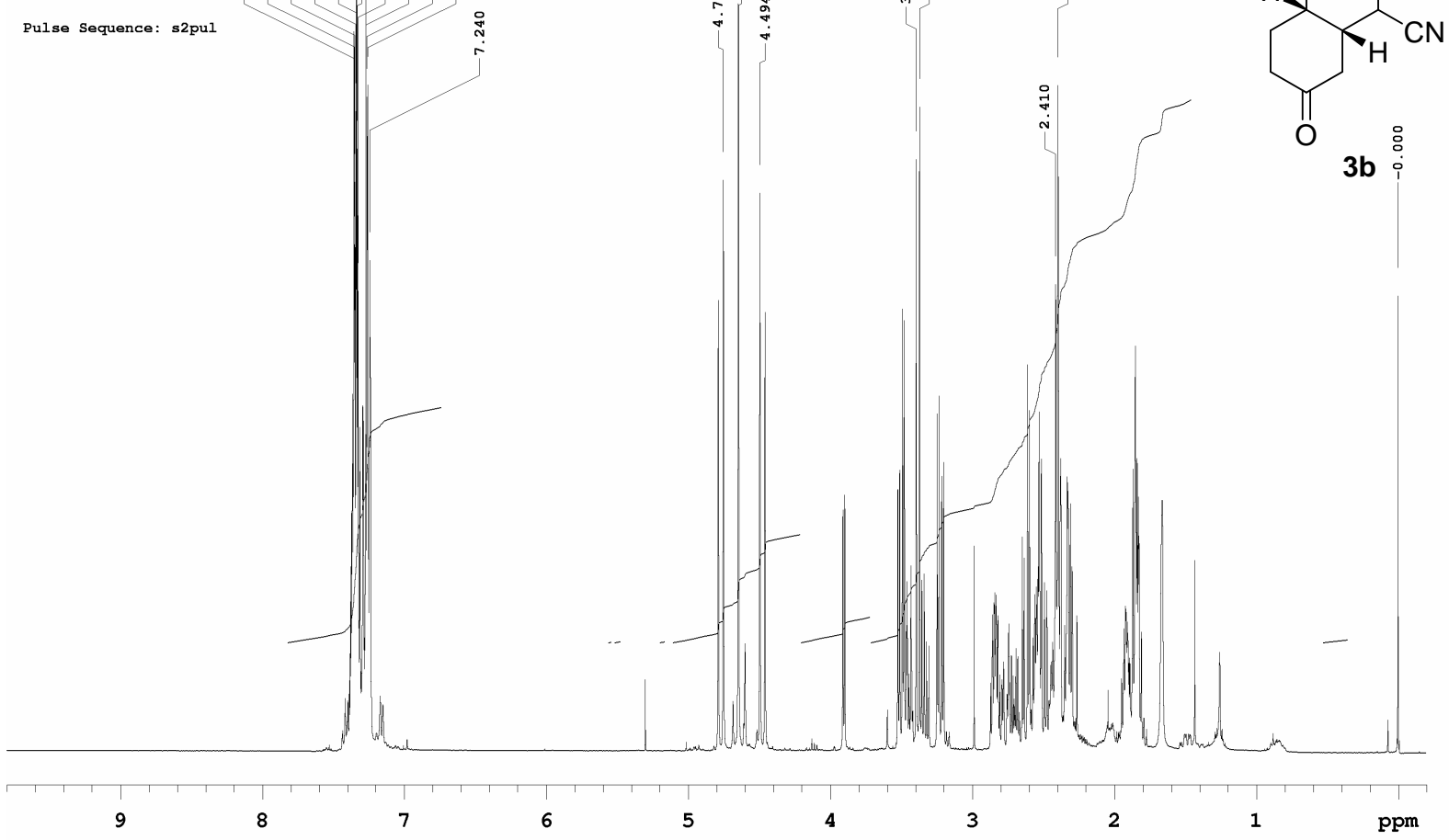

C13 / s2pul / Mercury-400_qui

edc13/Temp: 25C /N reg: M40004-221004164958

Usuar1: san / Mostra: 1p42312_

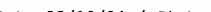

Pulse Sequence: s2pul
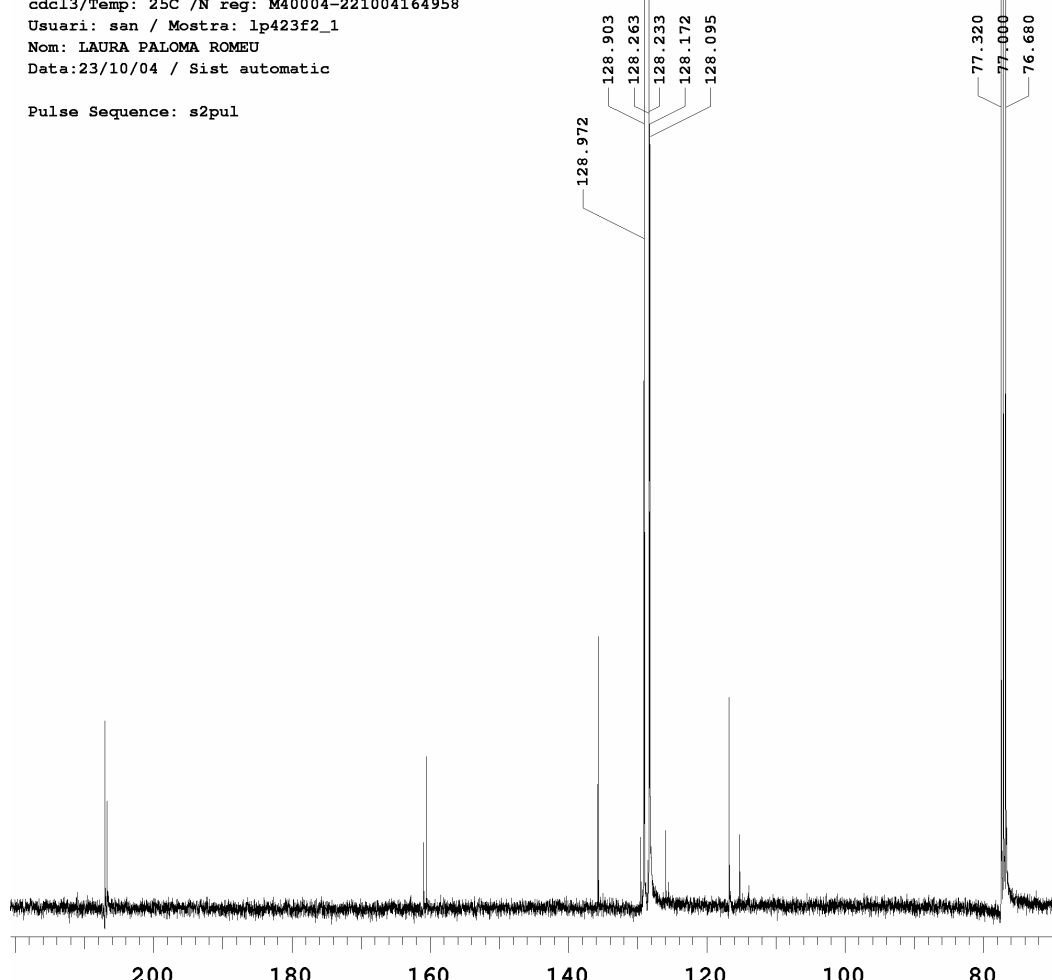

it

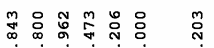

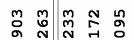

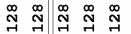

200

180

160

140

120

100

80

60

40

20

ppm 
H1 / gcosy / Mercury-400_qui cdc13/Temp: 25C /N reg: M40004-22100416495 Usuar1: san / Mostra: 1p423f2_1 Data:23/10/04/ Sist automatic

Pulse Sequence: gcosy<smiles>N#CC1C(=O)N(Cc2ccccc2)C[C@H]2CCC(=O)C[C@H]12</smiles>

$3 b$

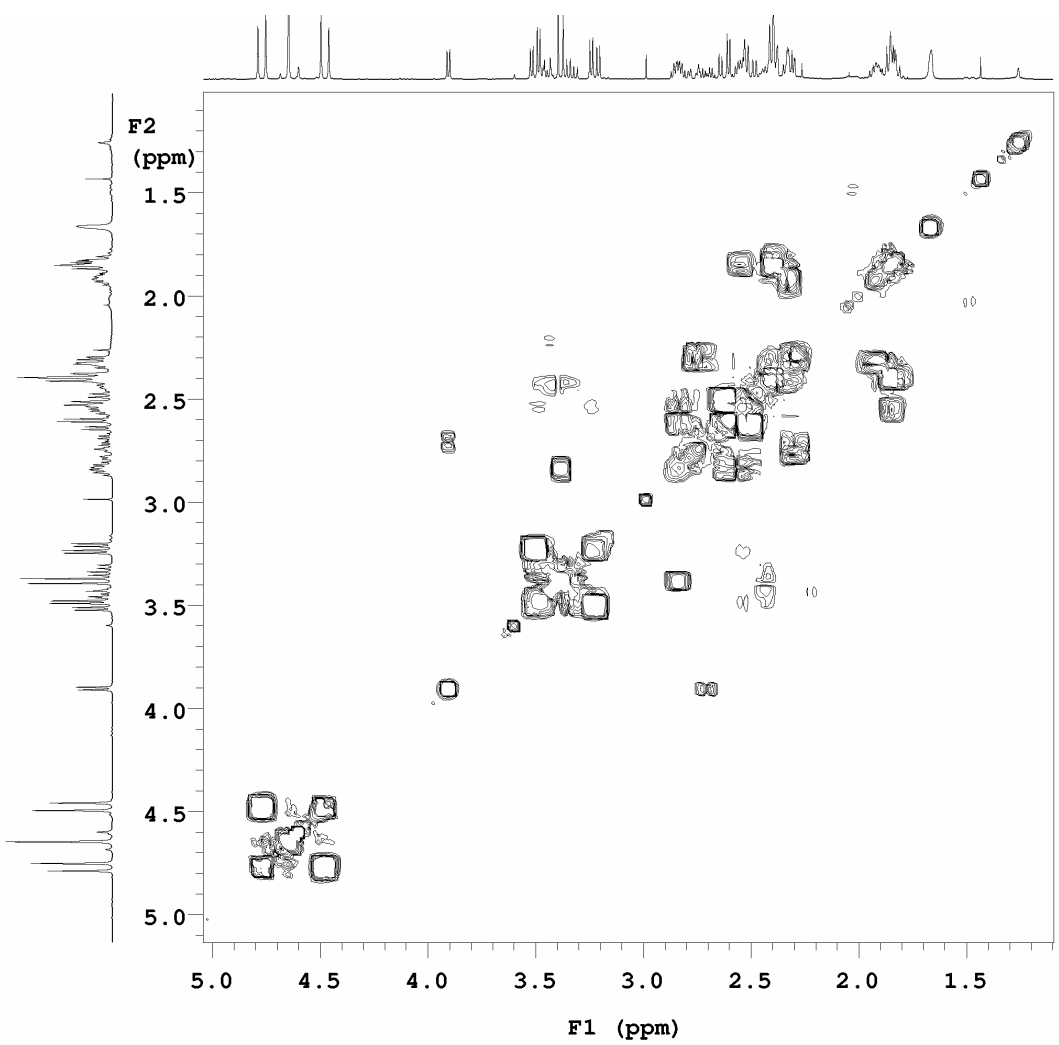

H1 / gHSQC / Mercury-400_qui
cdc13/Temp: 25C /N reg: M40004-22100416495 Usuari: san / Mostra: 1p423f2 Nom: LAURA PALOMA ROMEU

Data:23/10/04 / Sist automatic

Pulse Sequence: gHSQC<smiles>N#CC1C(=O)N(Cc2ccccc2)CC2CCC(=O)CC21</smiles>

$3 b$

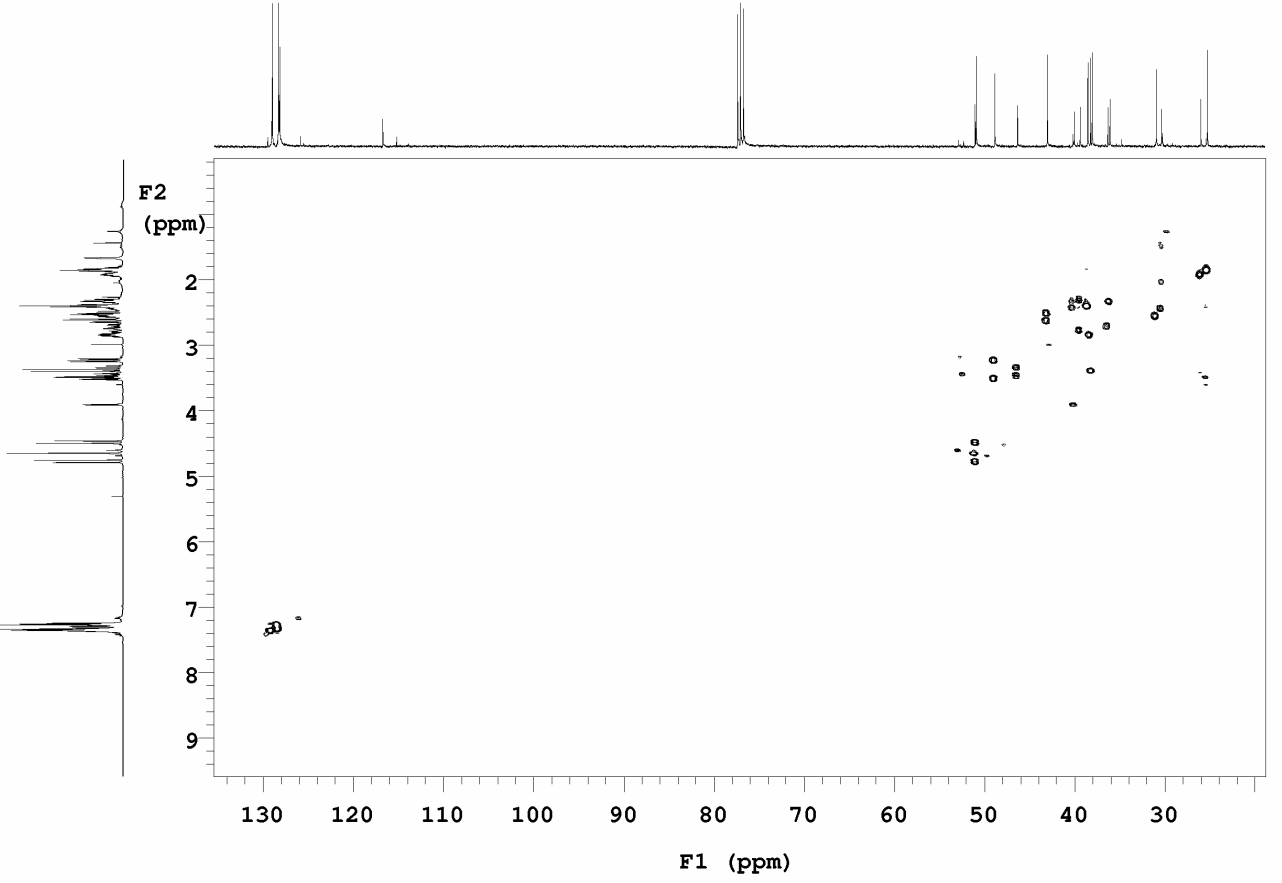


H1 / s2pul / Mercury-400_qui

cdc13/Temp: 25C /N reg: M40004-1227

Usuari: san / Mostra: 1p511

NOm: LAURA PALOMA ROMEU

Data:23/12/04 / Sist automatic

Pulse Sequence sapul

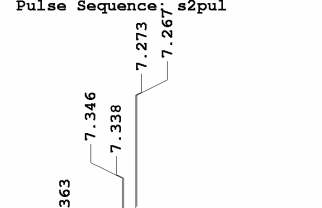

禺
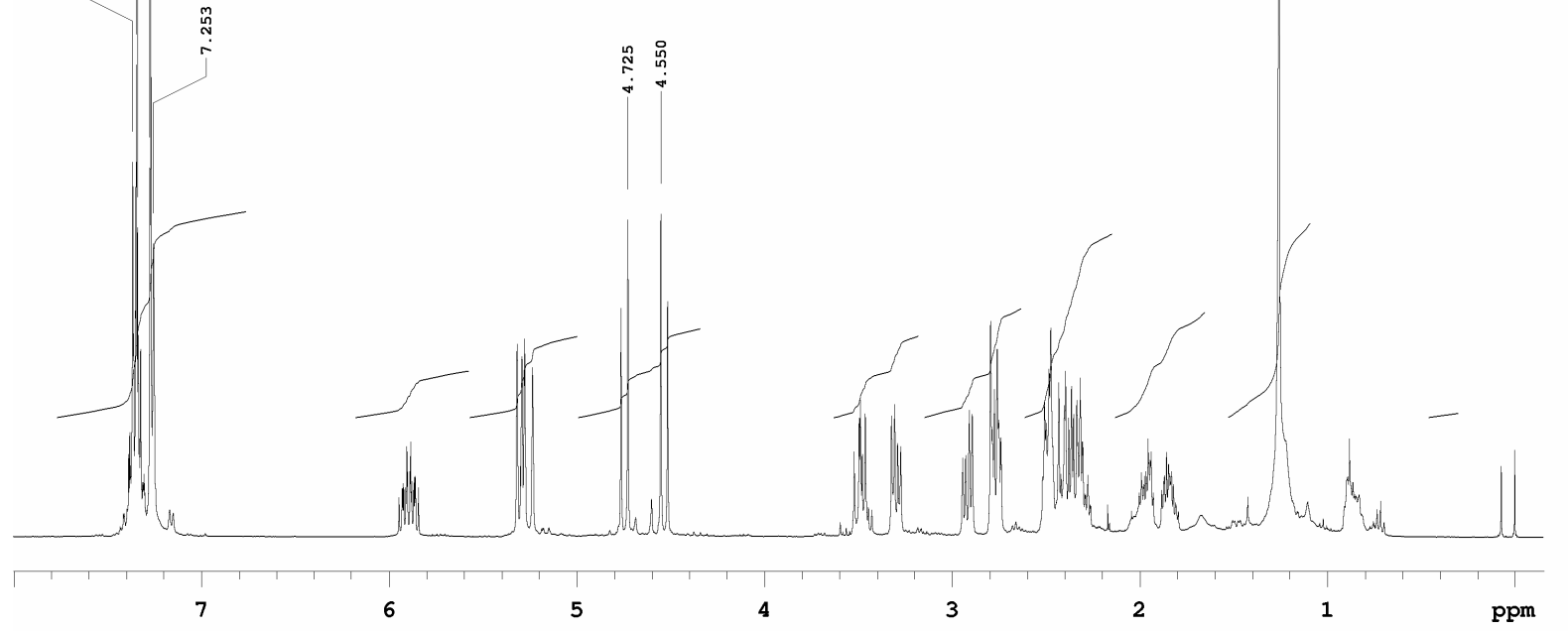

C13 / s2pul / Mercury-400 qui

cdc13/Temp: 25C/N reg: M40004-1227

Usuari: san / Mostra: lp511

NOM: LAURA PALOMA ROMEU

ํํㅇ 욱

Pulse Sequence: s2pul
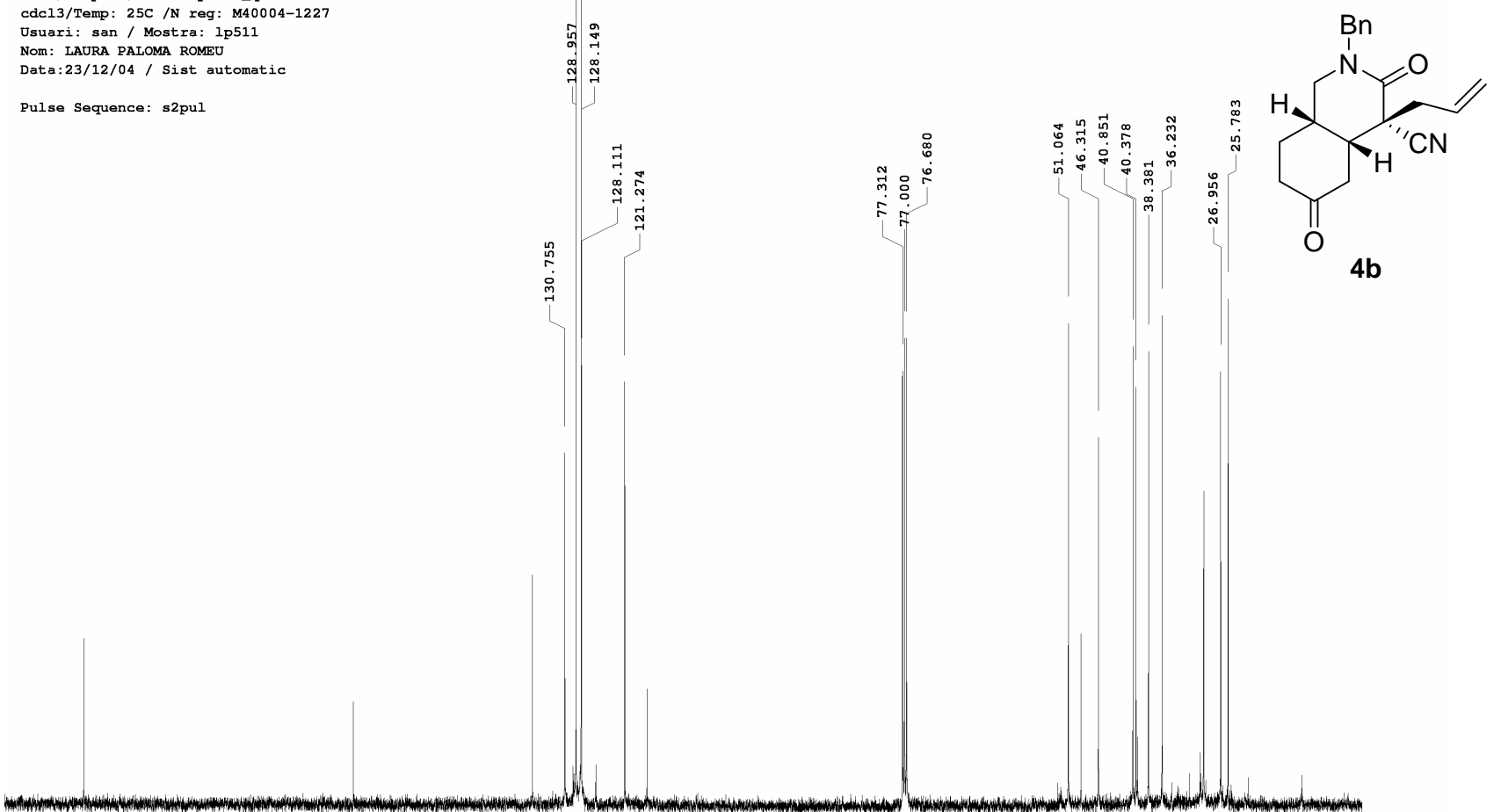

111

80

60

40

20 ppm 
H1 / gCOSY / Mercury-400_qui Ueg: M40004-1227 Usuri: san / Mostra: 1 p 511

Data: 23/12/04/Sist automatic

Pulse Sequence: gcosy<smiles>C=CC[C@]1(C#N)C(=O)N(Cc2ccccc2)C[C@H]2CCC(=O)C[C@H]21</smiles>

$4 b$

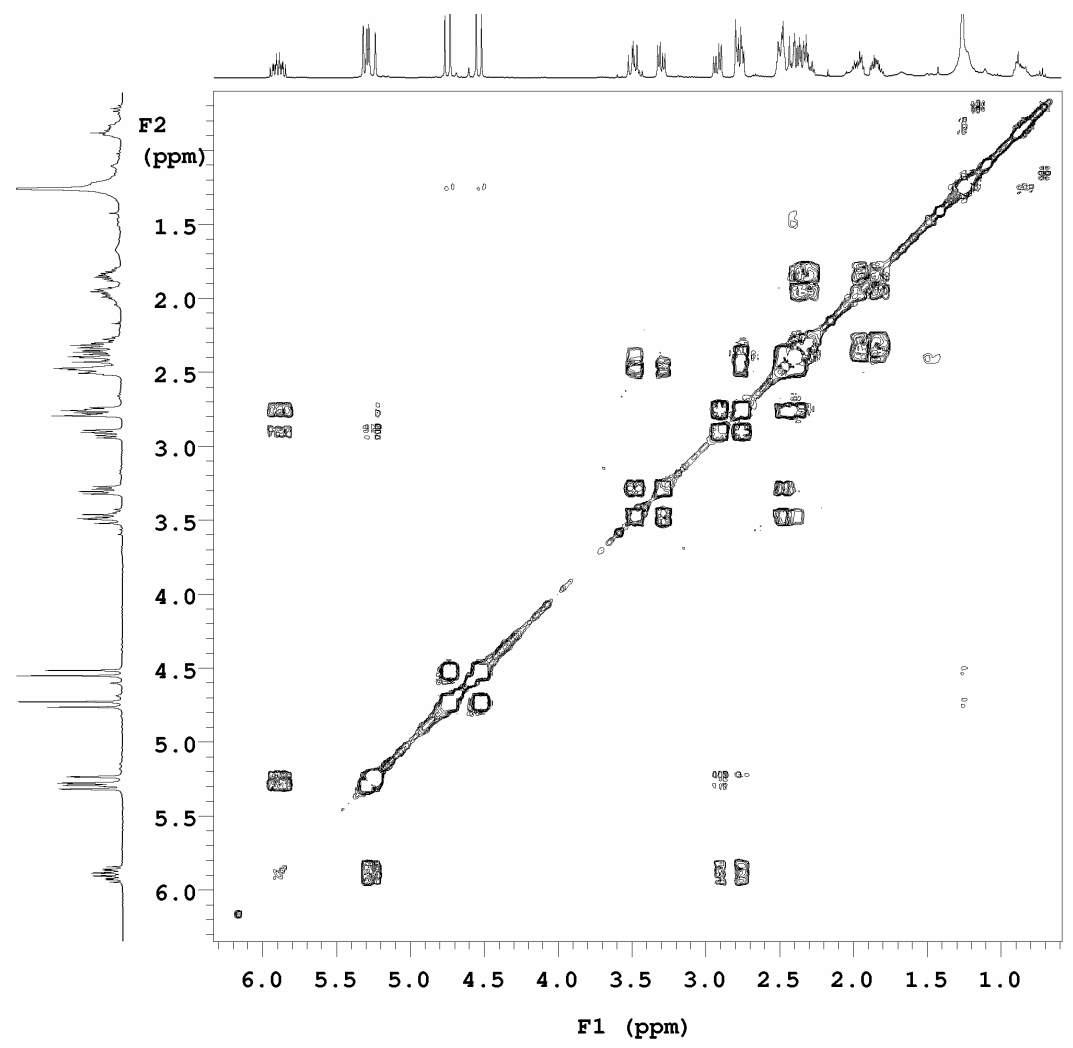

H1 / gHSQC / Mercury-400_qui

cdc13/Temp: $25 \mathrm{C} / \mathrm{N}$ reg: M40004-1227

Usuari: san / Mostra: 1p511

Data:23/12/04/ Sist automatic

Pulse Sequence: gHSOC<smiles>C=CC[C@]1(C)C(=O)N(Cc2ccccc2)C[C@H]2CCC(=O)C[C@H]21</smiles>

$4 b$

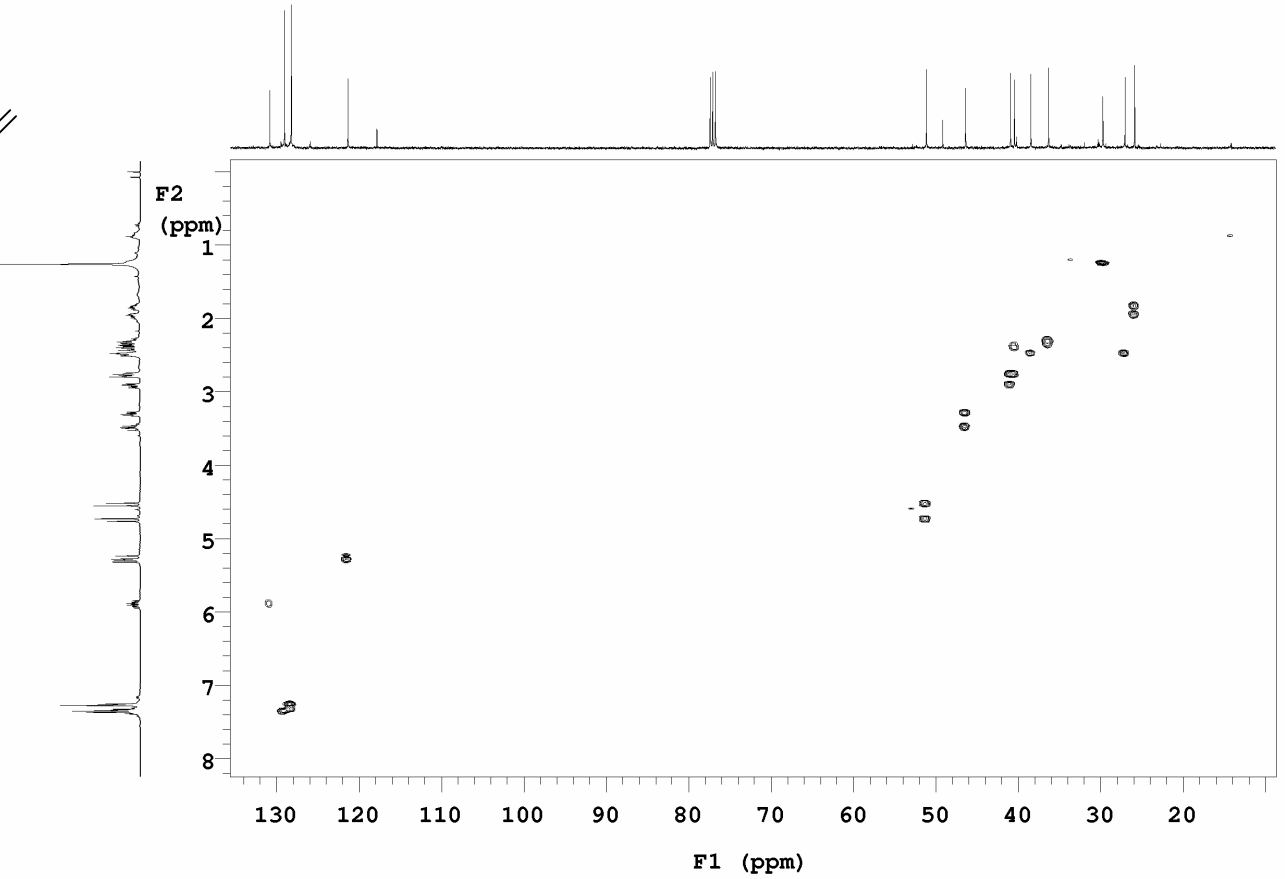



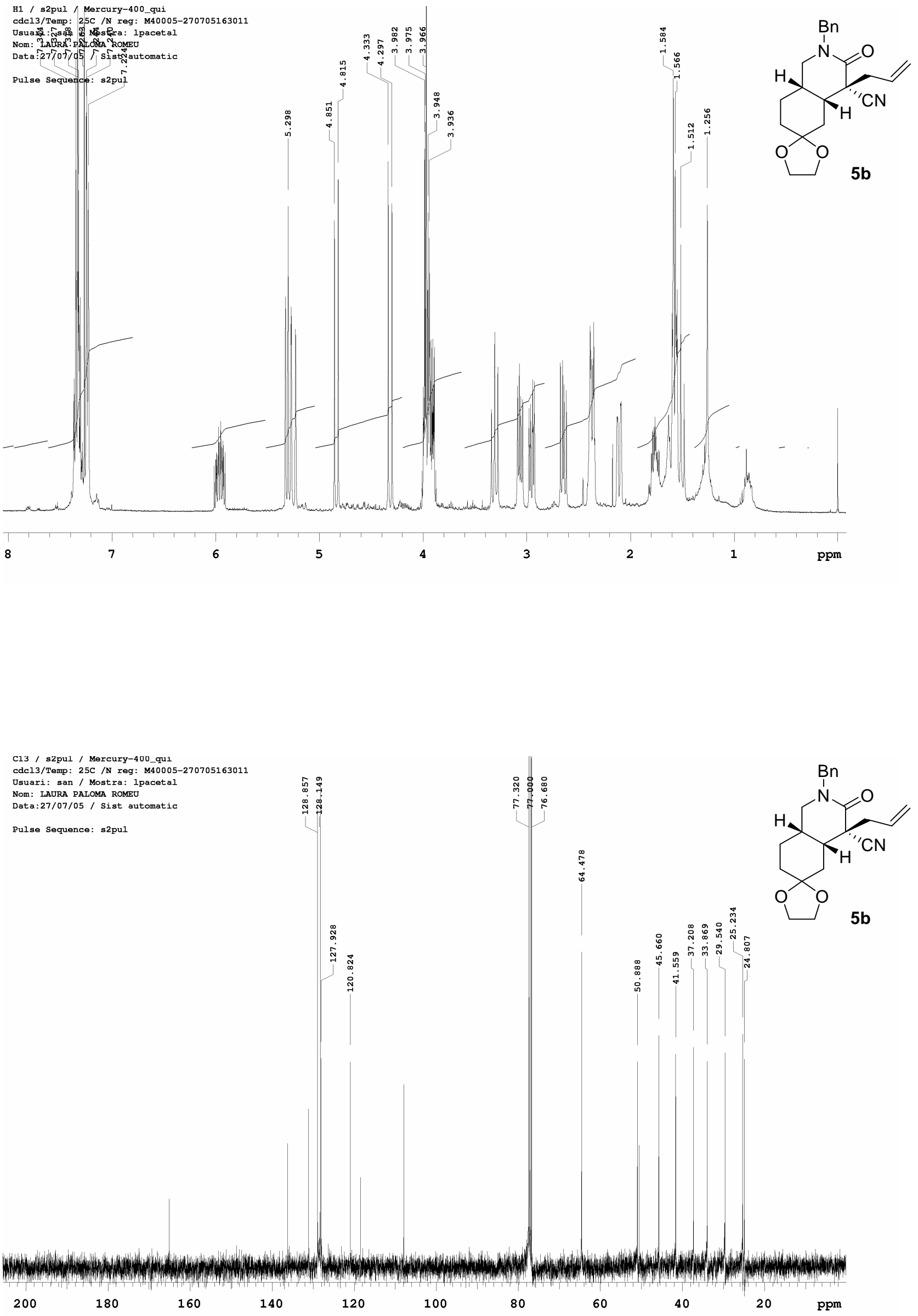
H1 / gCosy / Mercury-400_qui

$40005-270705163011$

Usuari: san / Mostra: 1pacetal

Data:27/07/05 / Sist automatic

Pulse Sequence: gcosy<smiles>C=CC[C@H]1C(=O)N(Cc2ccccc2)C[C@H]2CCC3(C[C@H]21)OCCO3</smiles>

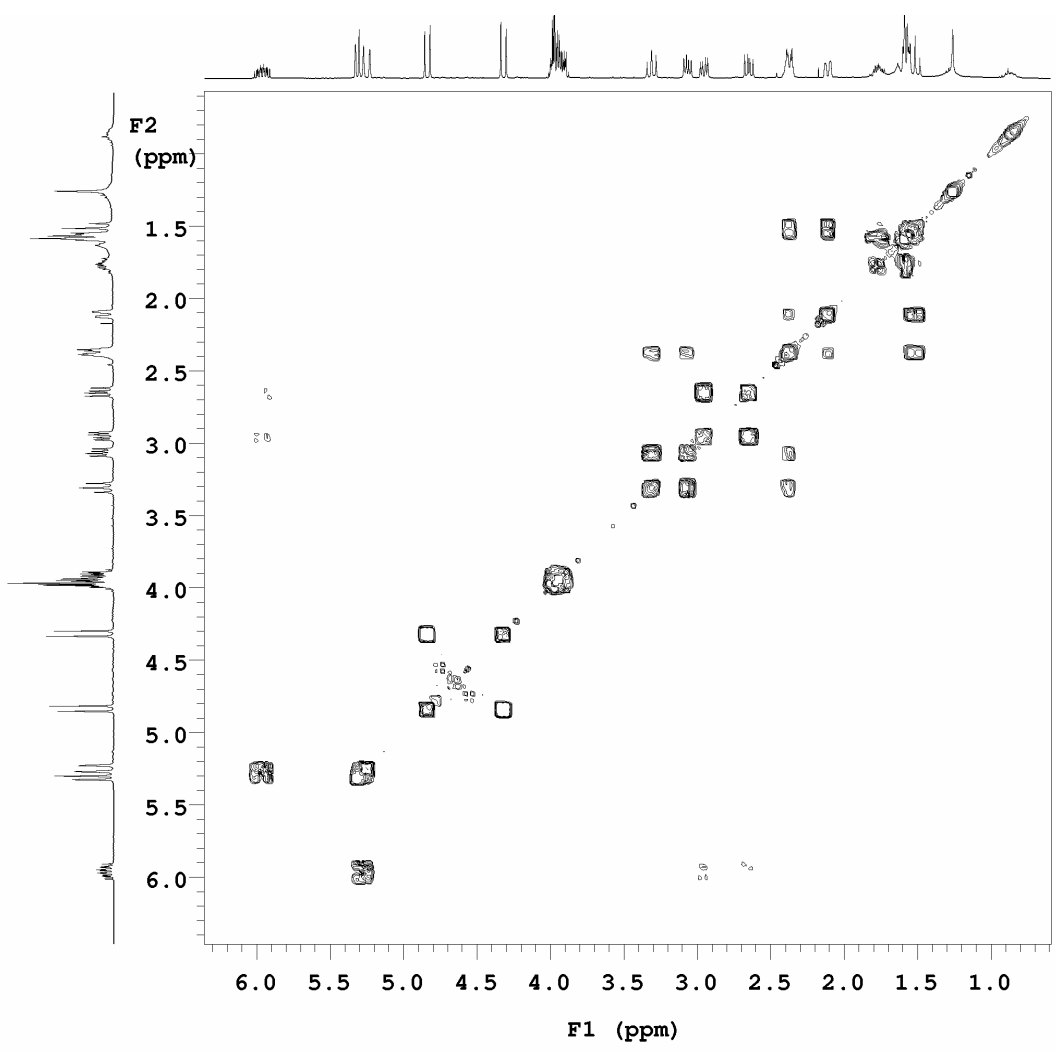

H1 / gHsQC / Mercury-400_qui

cdc13/Temp: 25C /N reg: M40005-270705163011

Usuari: san / Mostra: lpacetal

Nom: LAURA PALOMA ROMEU

Pulse Sequence: gHSOC

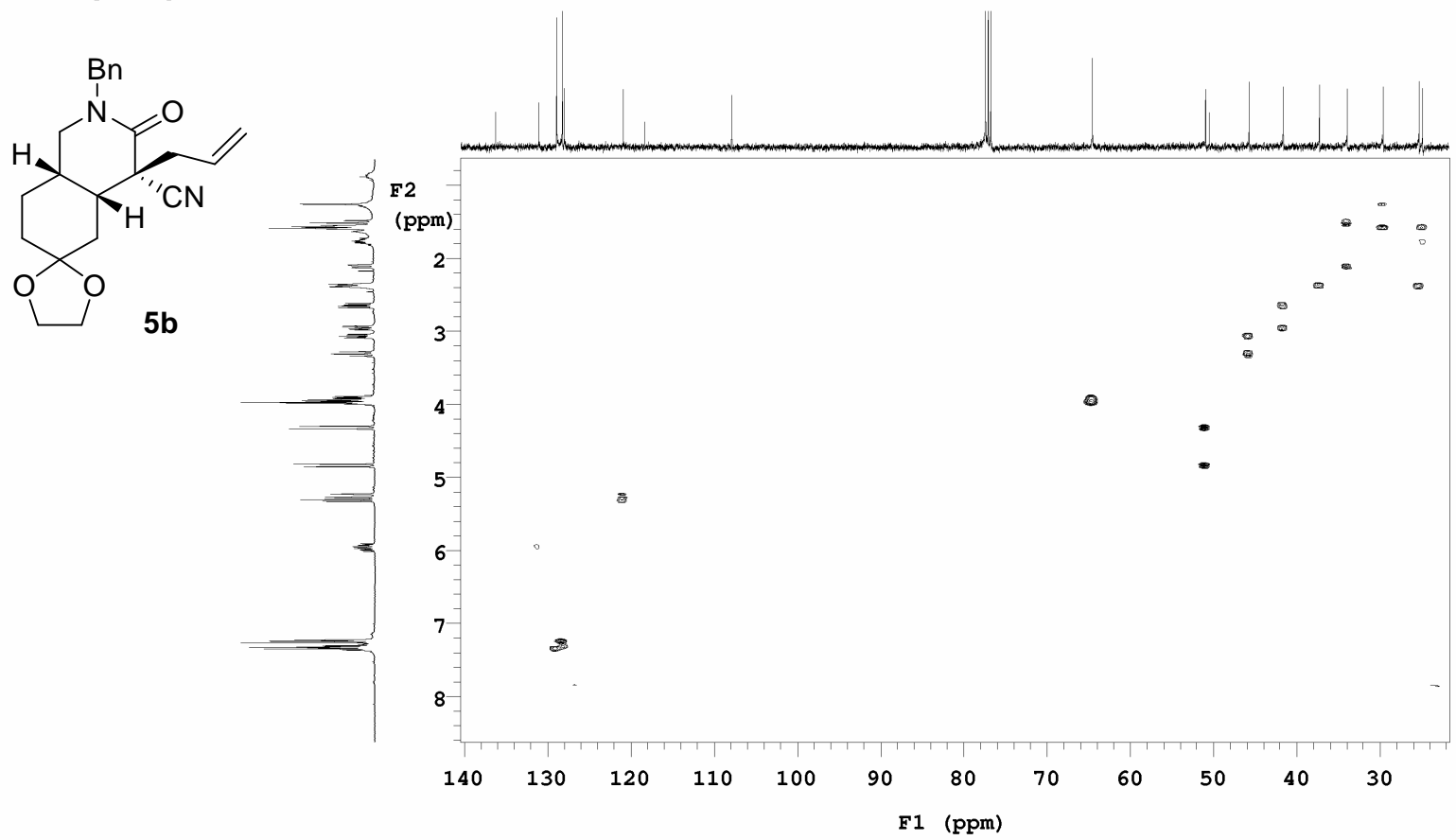


H1 / s2pul / Mercuypy-400F

cdc13 / Temp: 25C / N.Reg: $x x x x x x x x x x$

Usuari: $\operatorname{san} /$ sefseft

Data: 10/02/07 ODE.: ?I. PALOMA

Pulse Sequence: s2puI

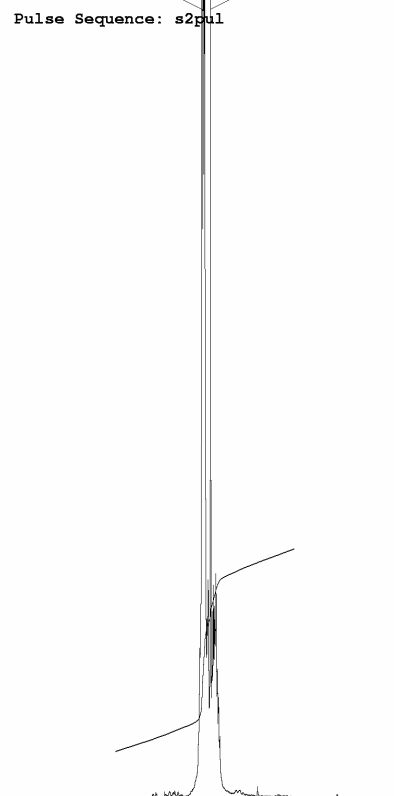

离

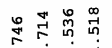

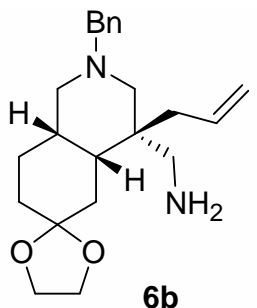

$6 b$

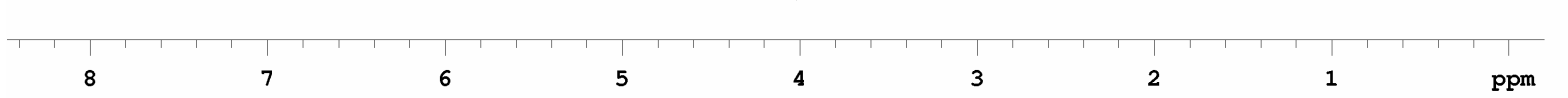

C13 / s2pul / Mercury-400F

cdc13 / Temp: 25C / N.Reg: $x x x x x x x x x x$

Usuari: san / Mostra: 1p1 pat

Nom: LAURA PALOMA ROMEU
Data: 10/02/07 / Ope.: L. RAM RAMA

Pulse Sequence: s2pul
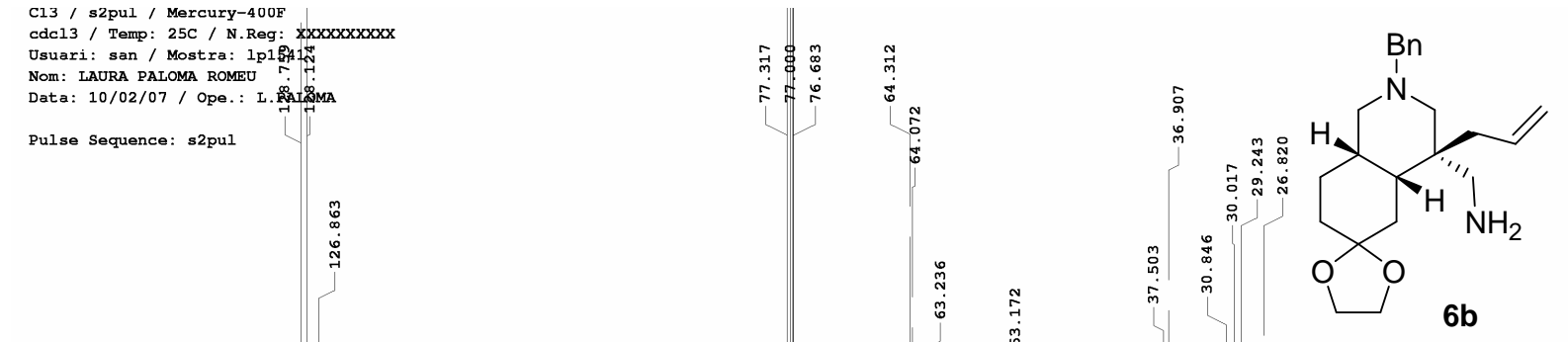

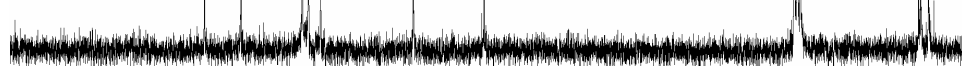


H1 / gCosY / Mercury-4008

/ $25 \mathrm{C} / \mathrm{N} \cdot \mathrm{Reg}: \mathrm{xxxxxxxxyx}$ Usuari: san / Mostra: 1p1541

Data: 10/02/07 / Ope.: L.PALOMA

Pulse Sequence: gcosy<smiles>C=CC[C@]1(CN)CN(Cc2ccccc2)CC2CCC3(CC21)OCCO3</smiles>

6b

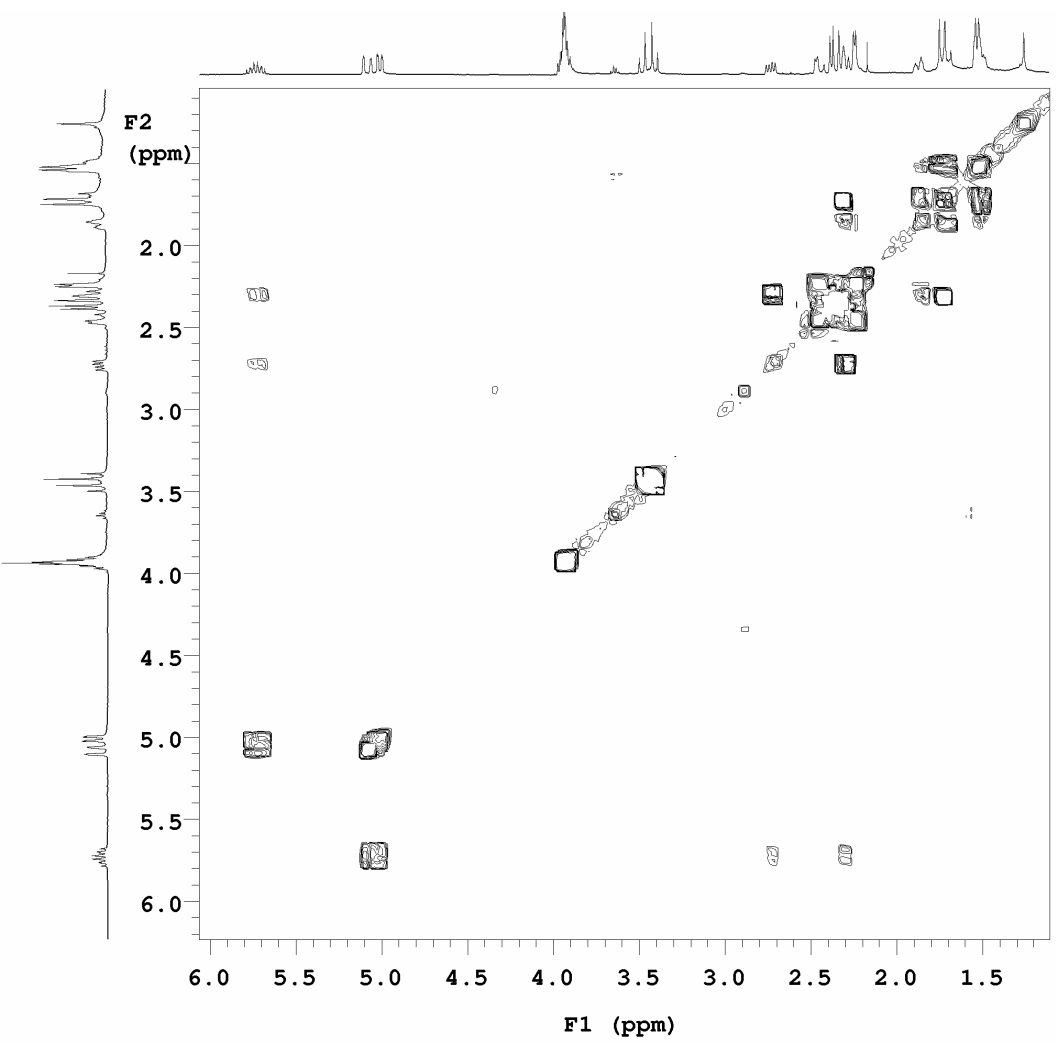

H1 / gHSOC / Mercury-400

cdc13 / Temp: 25C / N. Reg: $x x x x x x x x x x$

Usuari: san / Mostra: lp154

Nom: LAURA PALOMA ROMEU

Data: 10/02/07 / Ope.: L.PALOMA

Pulse Sequence: gHSOC<smiles>C=CC[C@]1(CN)CN(Cc2ccccc2)C[C@H]2CCC3(C[C@H]21)OCCO3</smiles>

6b

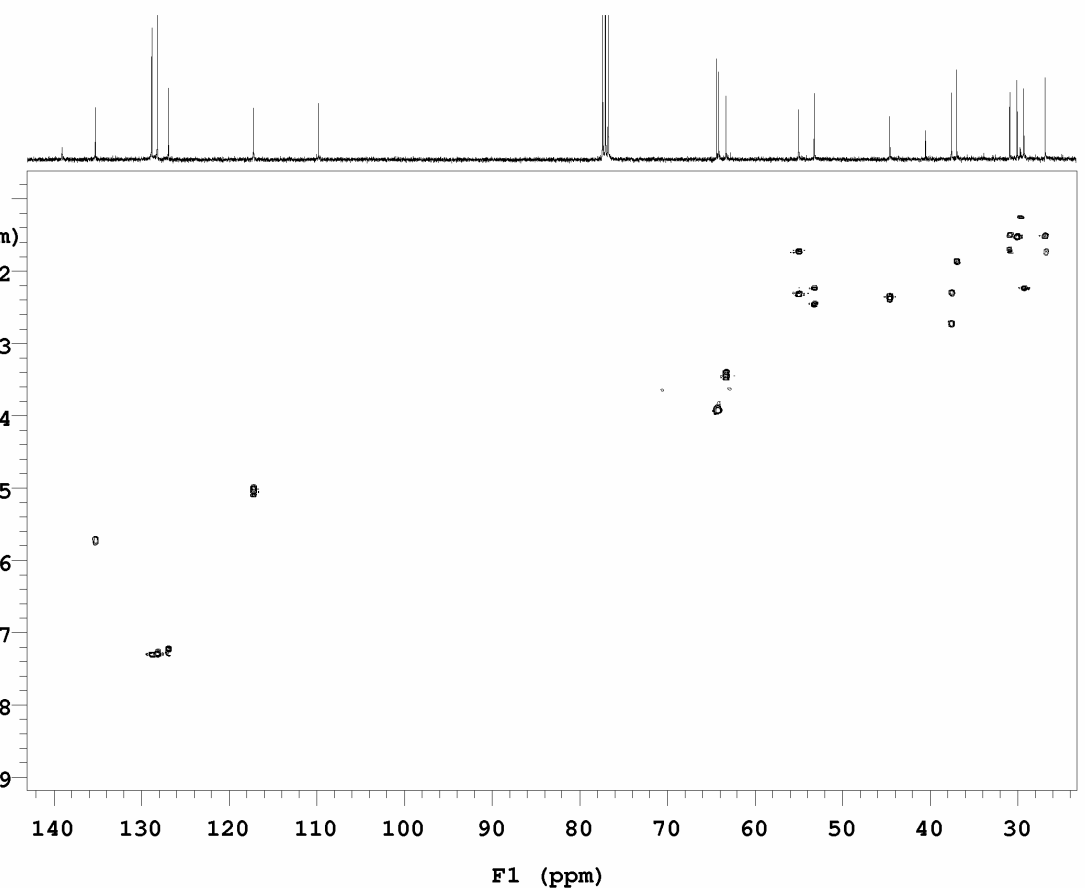




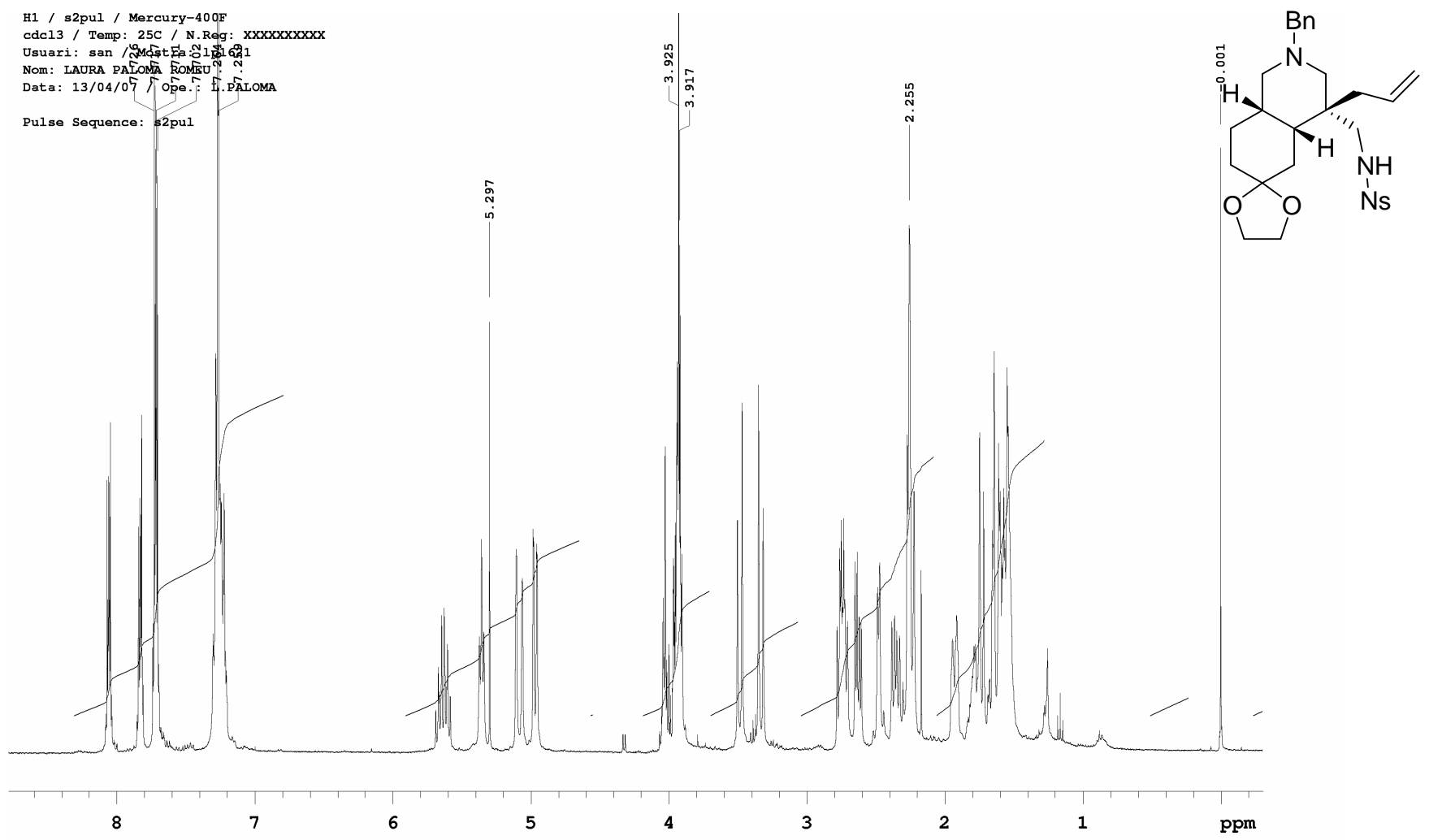

C13 / s2pul / Mercury-400F

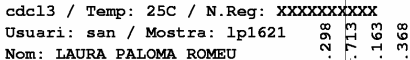

Won: LATA PALOMA ROMEU

13/04/07/Ope.: L.PALOMA m

m

离

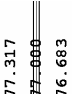

$\stackrel{20}{2}$

Pulse Sequence: s2pul

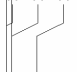

L

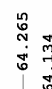

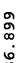
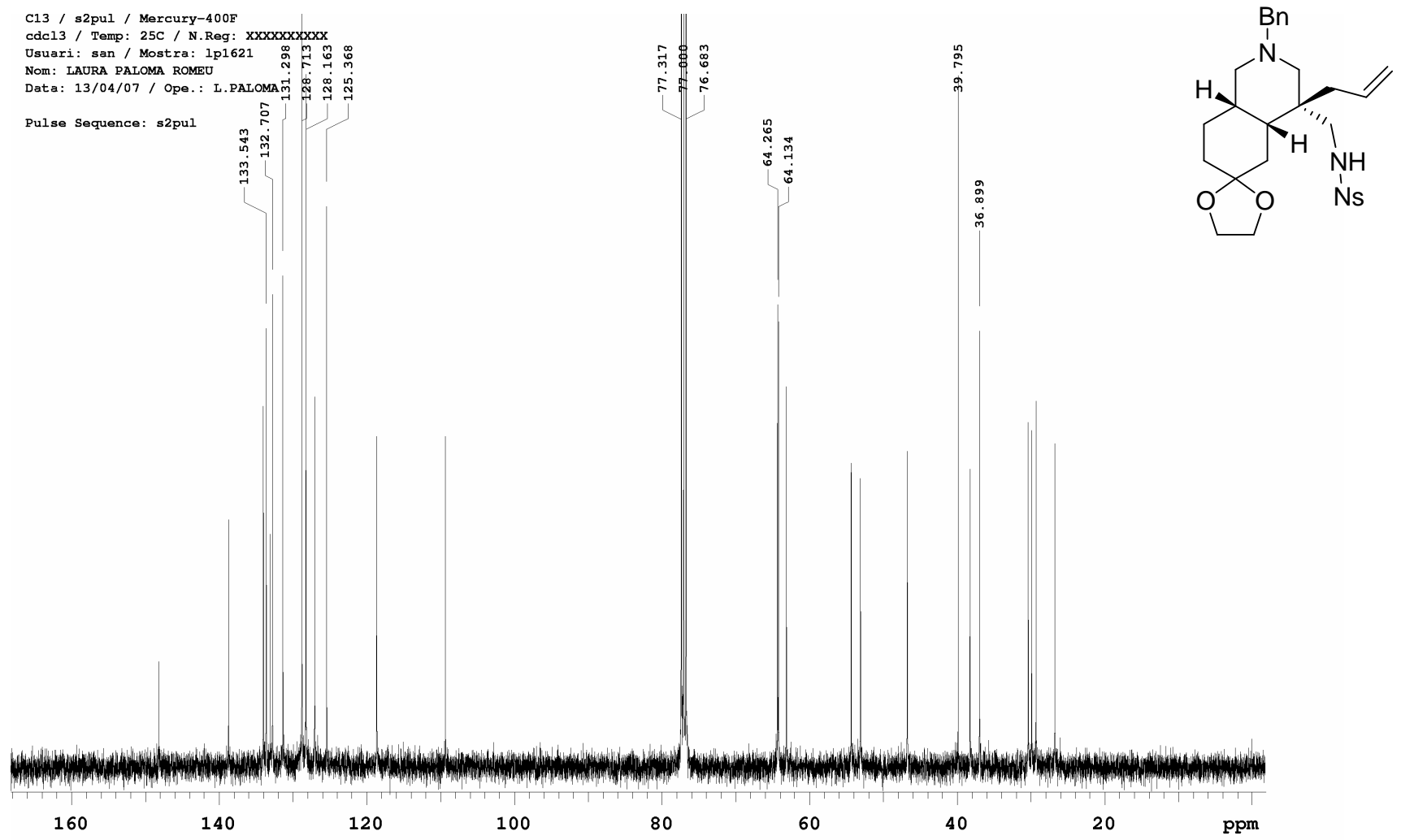
H1 / gCosY / Mercury-400F cdc13 / Temp: 25C / N.Reg: $\mathrm{xxxxxxxxxx}$ Usuari: san / Mostra: 1p1621 Nom: LAURA PALOMA ROMEU Pulse Sequence: gcosy<smiles>C=CC[C@H]1CN(Cc2ccccc2)C[C@@H]2CCC3(C[C@H]12)OCCO3</smiles>

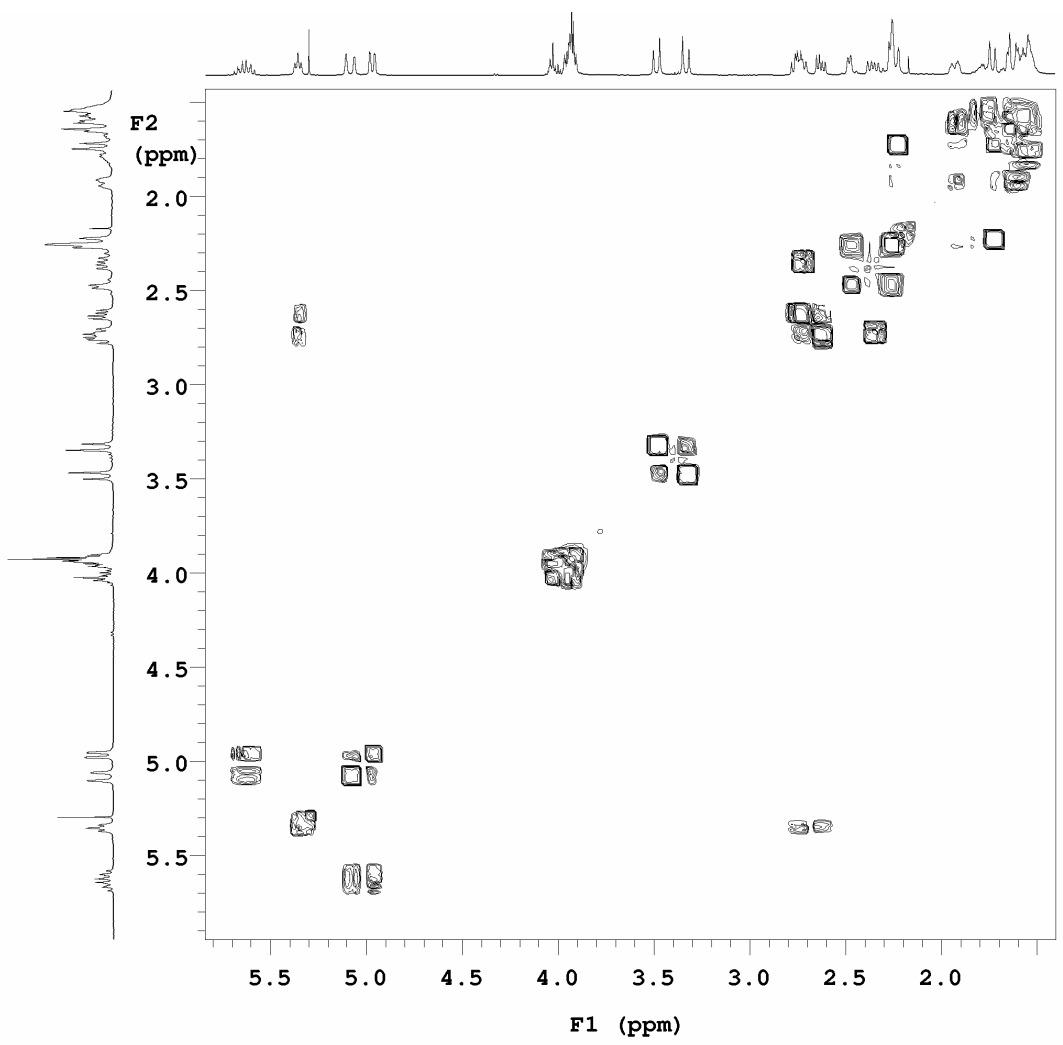

H1 / gHSQC / Mercury-400F

dc13 / Temp: 25C / N.Reg: xxxxxxxxxx

Domitison / Mostra: 1p1621

Pulse Sequence: gHSOC<smiles>C=CC[C@H]1CN(Cc2ccccc2)C[C@@H]2CCC3(C[C@H]12)OCCO3</smiles>

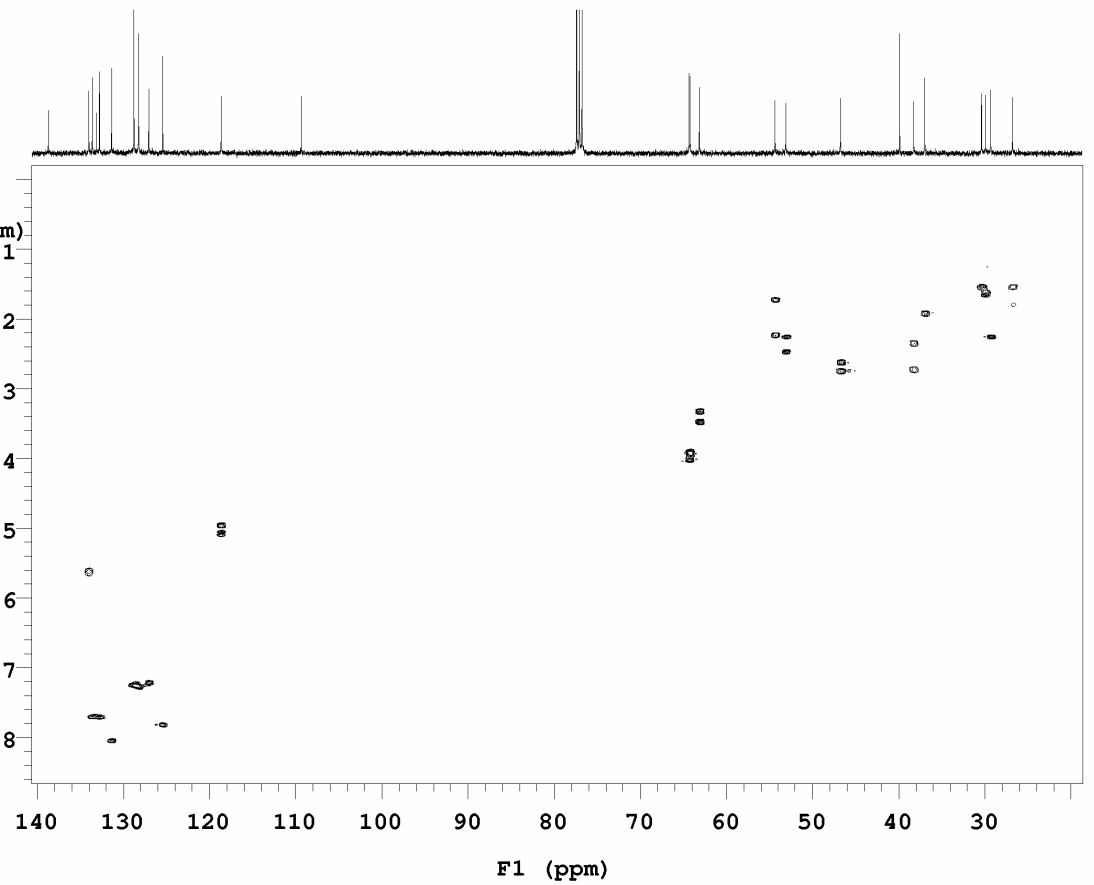




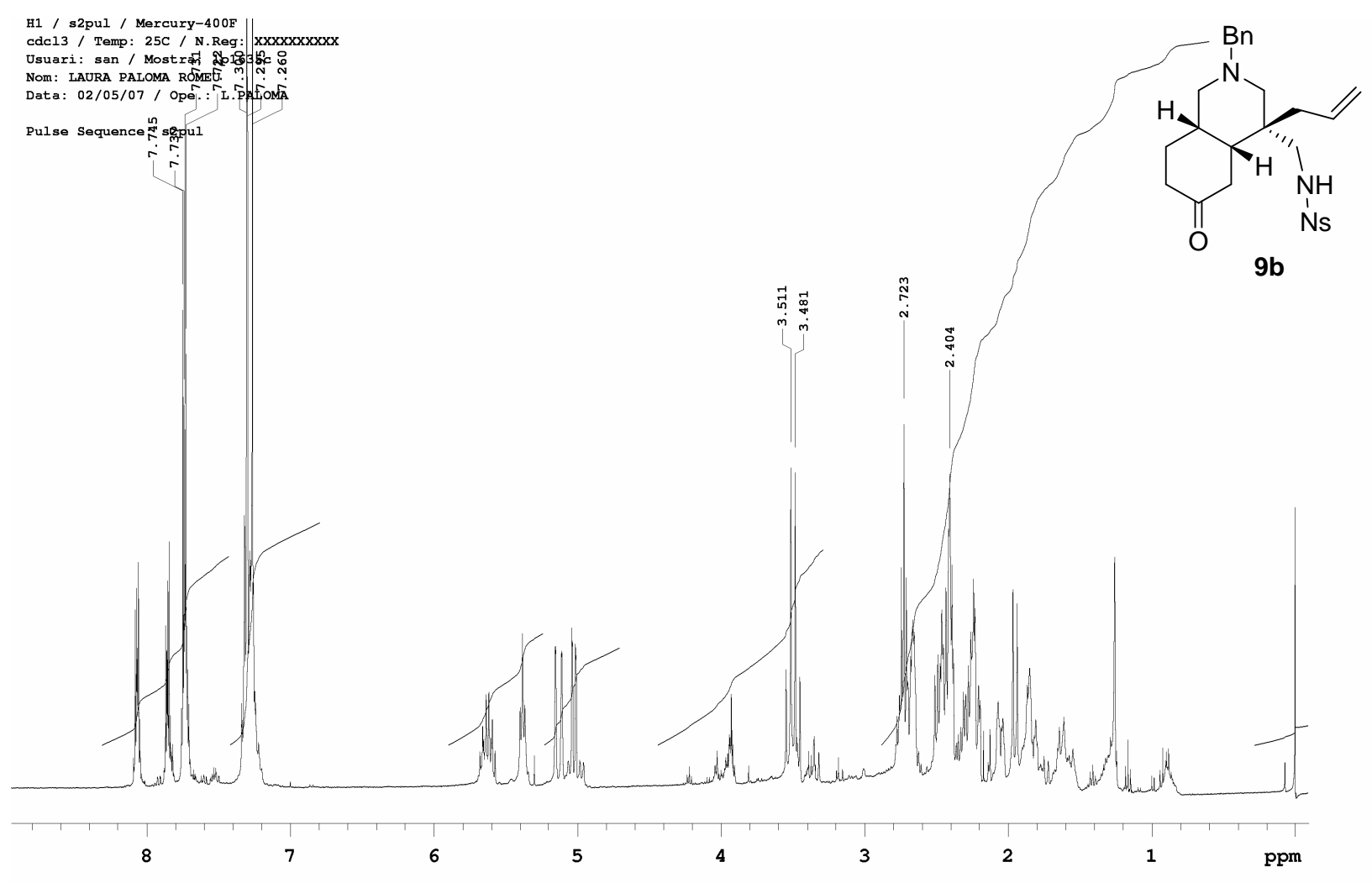

C13 / s2pul / Mercury-400F

cdc13 / Temp: 25C / N.Reg: $x x x x x x x x x x$

Usuari: san / Mostra: $1_{p}$

OM: LAURA PALOMA ROM

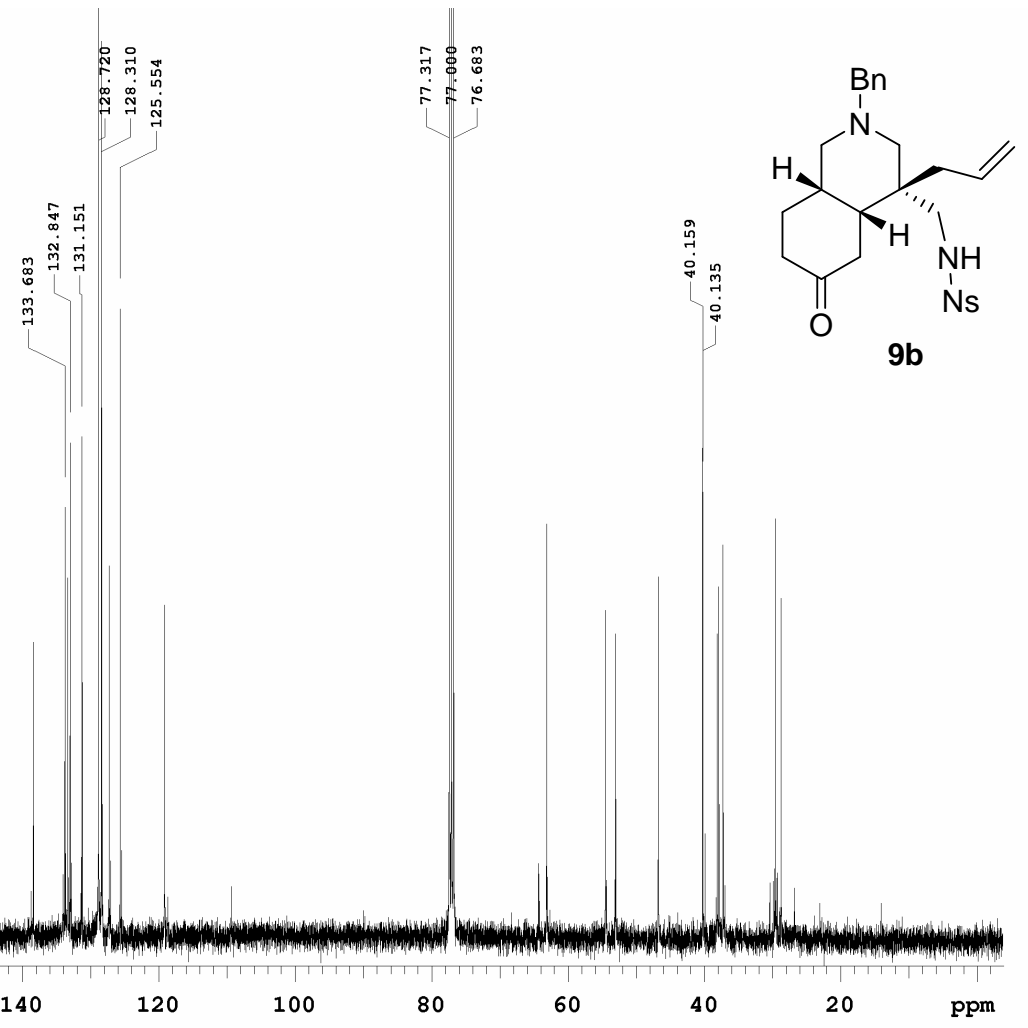


H1 / gCosY / Mercury-400F

cdc13 / Temp: $25 \mathrm{C} / \mathrm{N} \cdot \operatorname{Reg}: \mathrm{xxxxxxxxxx}$ Usuari: san / Mostra: 1p1635

Pulse Sequence: gcosY<smiles>C=CC[C@H]1CN(Br)C[C@H]2CCC(=O)C[C@@H]21</smiles>

$9 b$

H1 / gHSQC / Mercury-400

N.Reg: $x x x x x x x x x x$

Data: $02 / 05 / 07 /$ Ope : L. PALOMA

Pulse Sequence: gHSOC<smiles>C=CC[C@]1(CNN)CN(Cc2ccccc2)C[C@H]2CCC(=O)C[C@H]21</smiles>

$9 b$

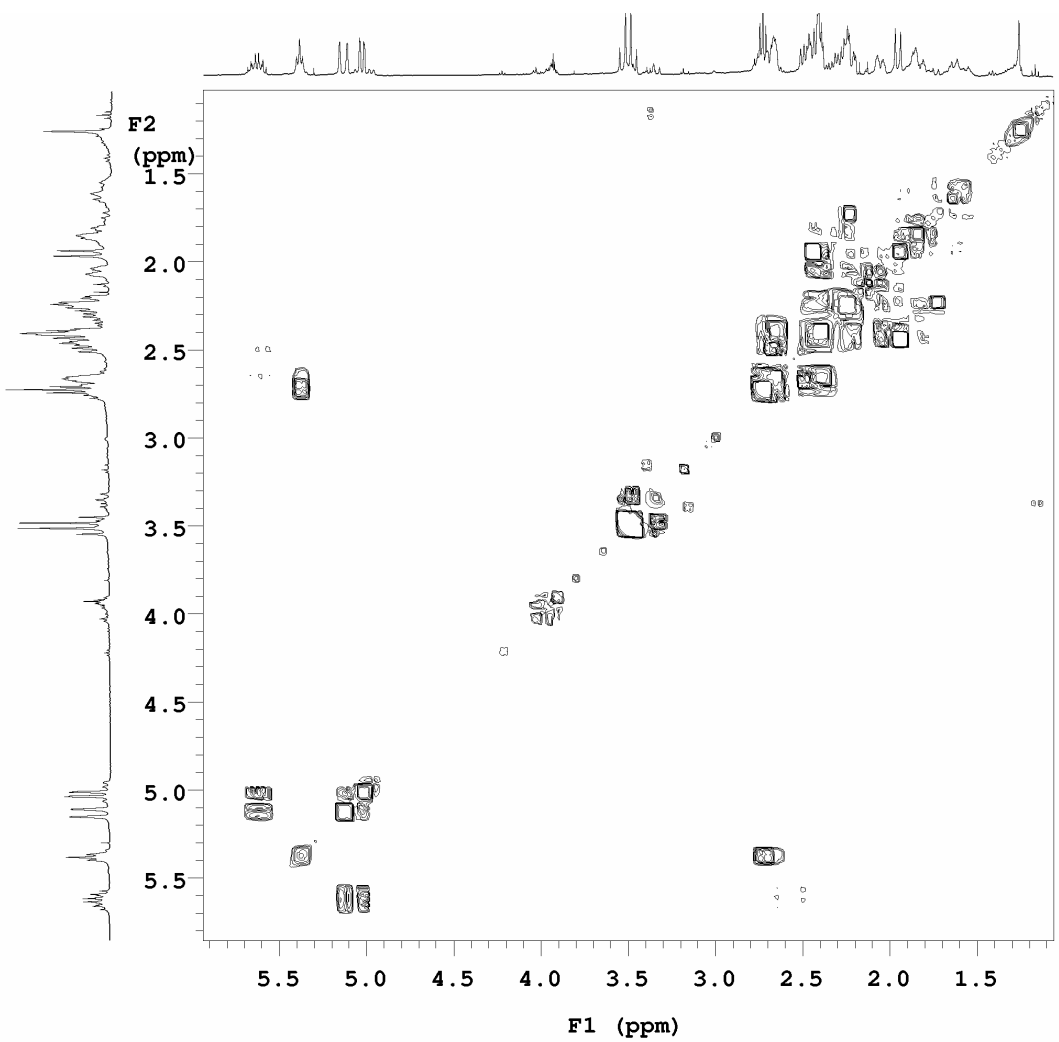

F1 (ppm) 


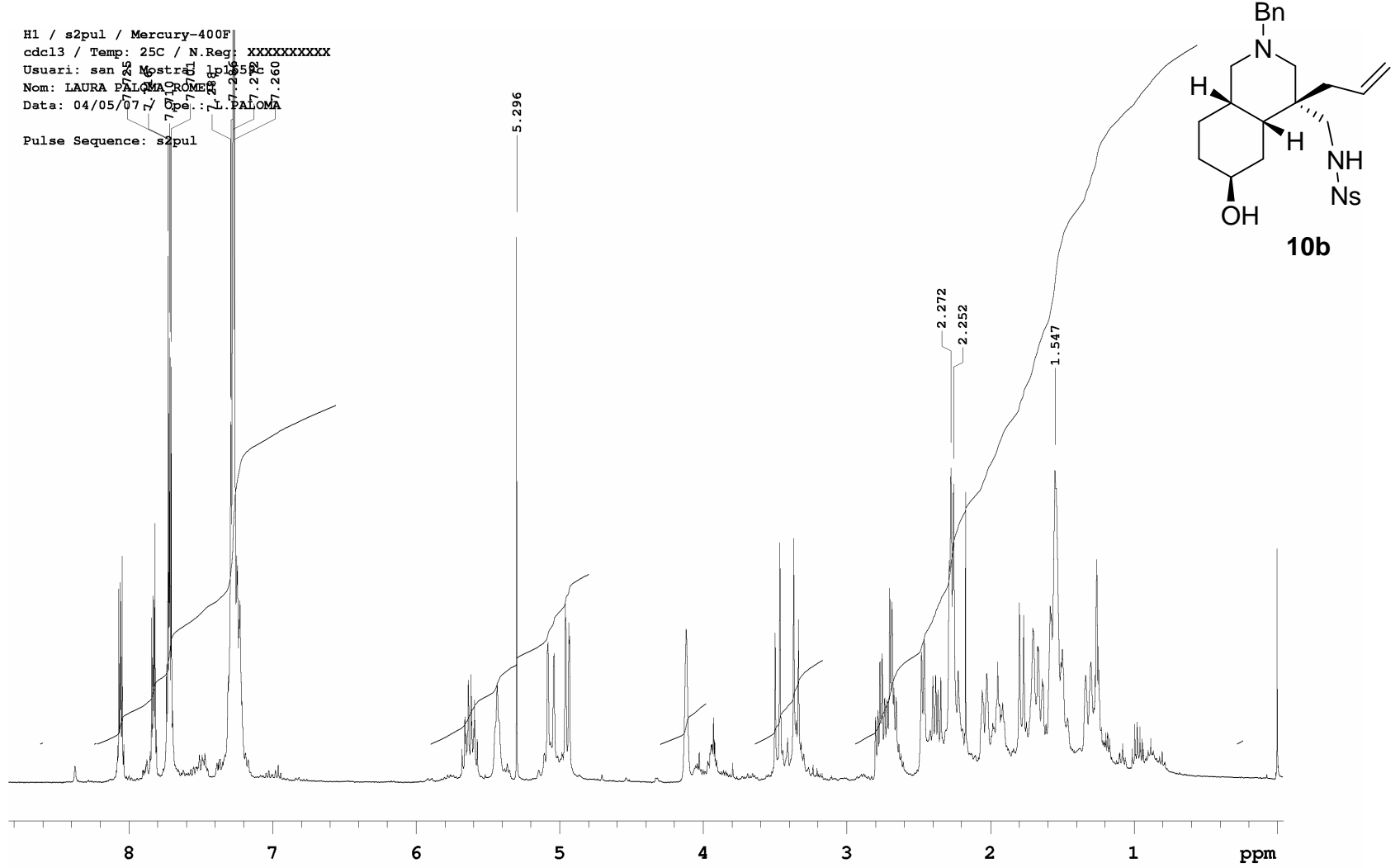

C13 / s2pul / Mercury-400F

cdc13 / Temp: 25c/ N.Reg: $x x x x x x y x y x$

Nom:

Data: 04/05/07/Ope.: L.PALOM⿻

Pulse Sequence: s2pul
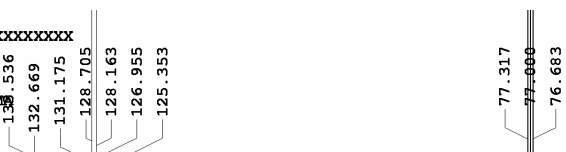

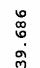

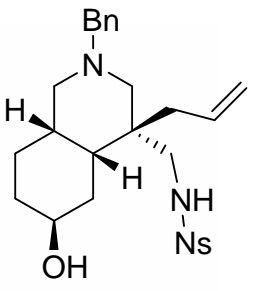

$10 b$

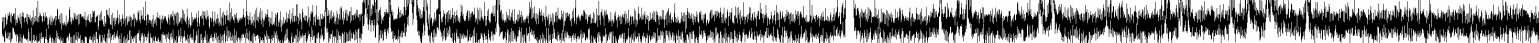


H1 / gCOsY / Mercury-400F

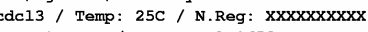
Dsuari: san / Mostra: Non: 204 /05/07/ Opa : L. PALOMA 11 data ore in file Hl

Pulse Sequence: gcosY<smiles>C=CC[C@]1(CNN)CN(Cc2ccccc2)CC2CCC(O)C[C@H]21</smiles>

$10 \mathrm{~b}$

H1 / gHSQC / Mercury-400

cdc13 / Temp: 25C / N.Reg: $\mathrm{xxxxxxxxxx}$

Usuari: san / Mostra: 1p1659c

Nom: LAORA PALOMA RI

Pulse Sequence: gHSQC<smiles>C=CC[C@]1(CNN)CN(Cc2ccccc2)C[C@H]2CC[C@H](O)C[C@H]21</smiles>

$10 \mathrm{~b}$

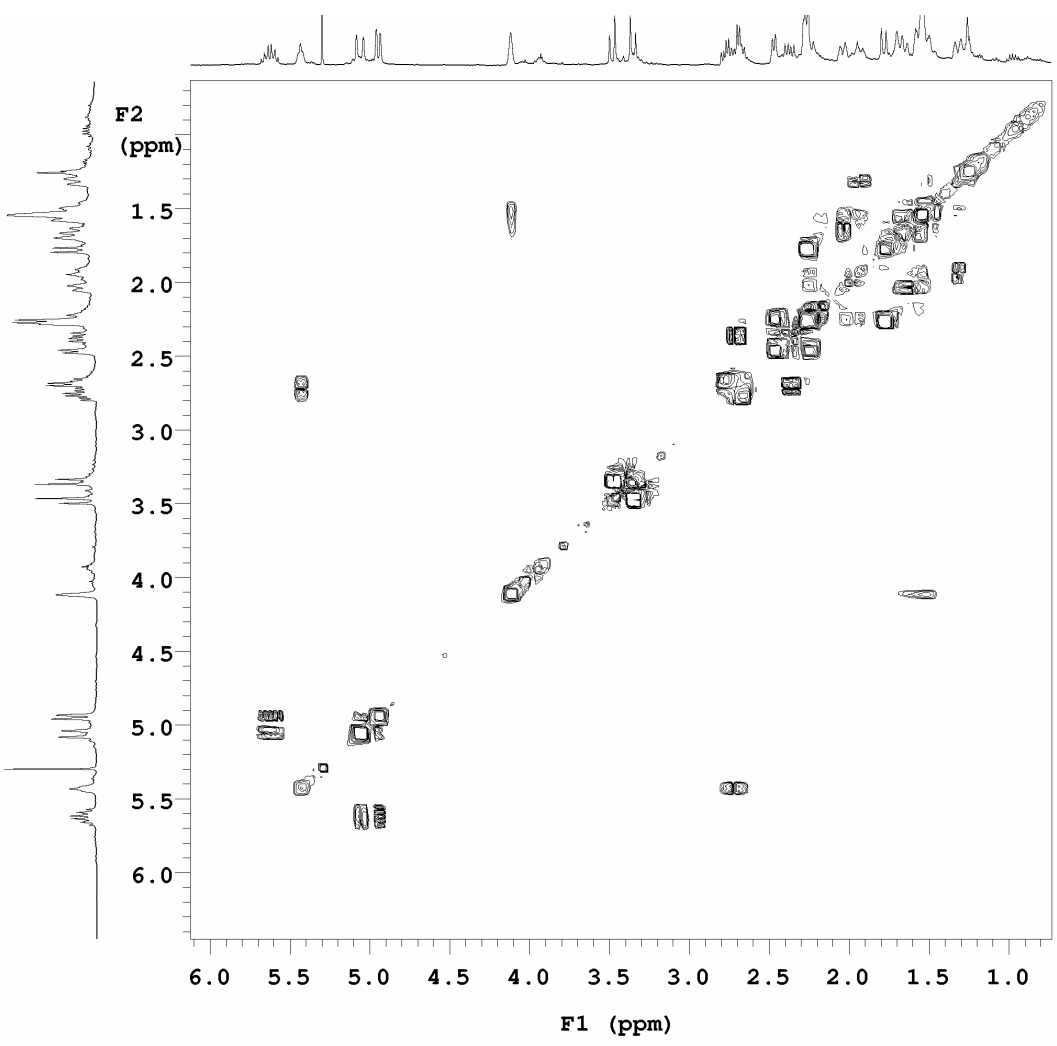


H1 / s2pul / Mercury+400F

cdc13/Temp: 25C/N.Reg: xxxxxxxxxx

Dsuari:

Dom:

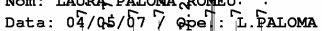

pulse sequence: s2 puil
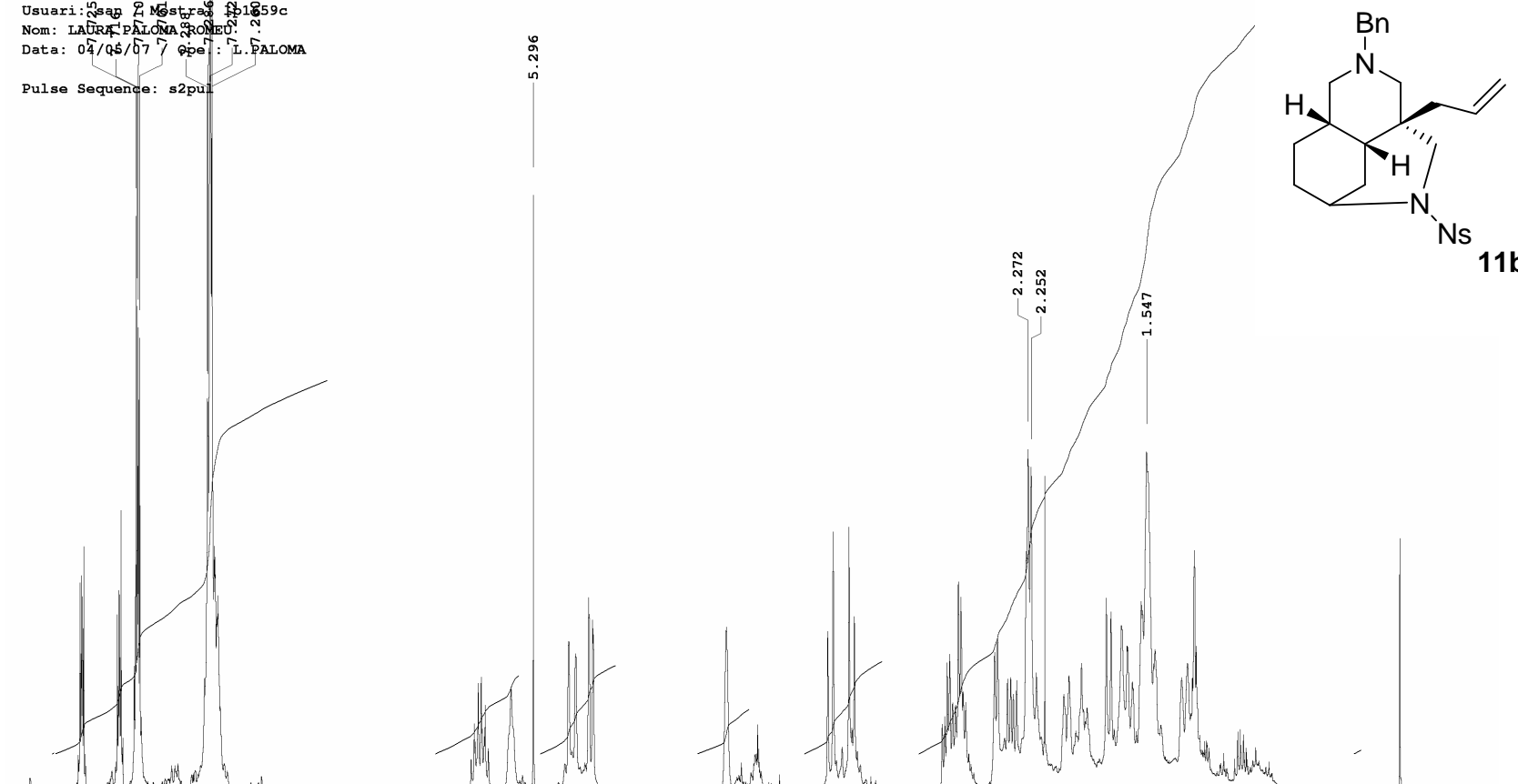

11b

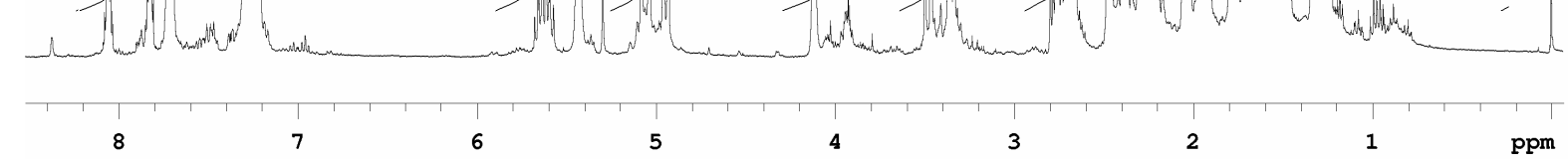

C13 / s2pul / Mercury-400F

Usuari: san / Mostra: 1p1631 స్ర

Data: 21/04/07 / Ope.: L. PALLMAN

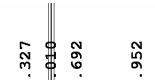

Pulse Sequence: s2pul

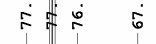

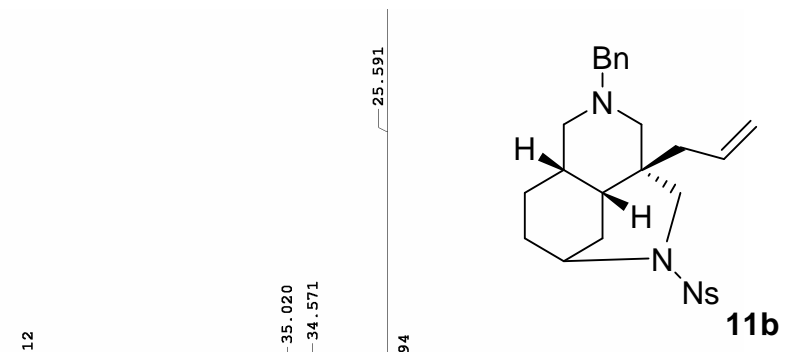

:

in

$11 b$

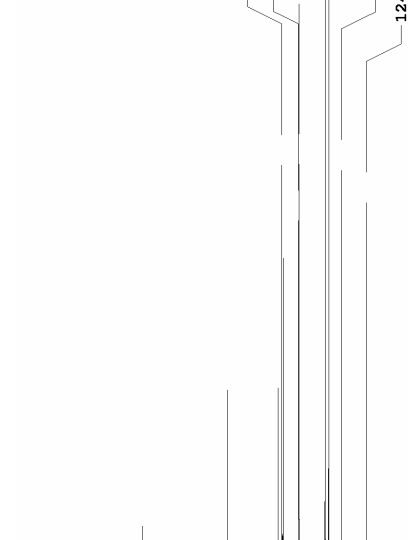

द

玄 
H1 / gCosy / Mercury-400F

cdc13 / Temp: 25C / N.Reg: xxxxxxxxxx

Usuari: san / Mostra: Ip1631

Nom: LAURA PALOMA ROMEU

Data: 21/04/07 / Ope.: L. PALOMA

Pulse Sequence: gcosY

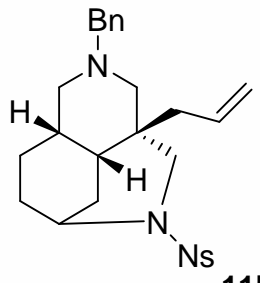

$11 b$

H1 / gHSQC / Mercury-400F

Reg: $x x x x x x x x x x$

(1p163

Data: 21/04/07 / Ope.: L.PALOMA

Pulse Sequence: gHSOC

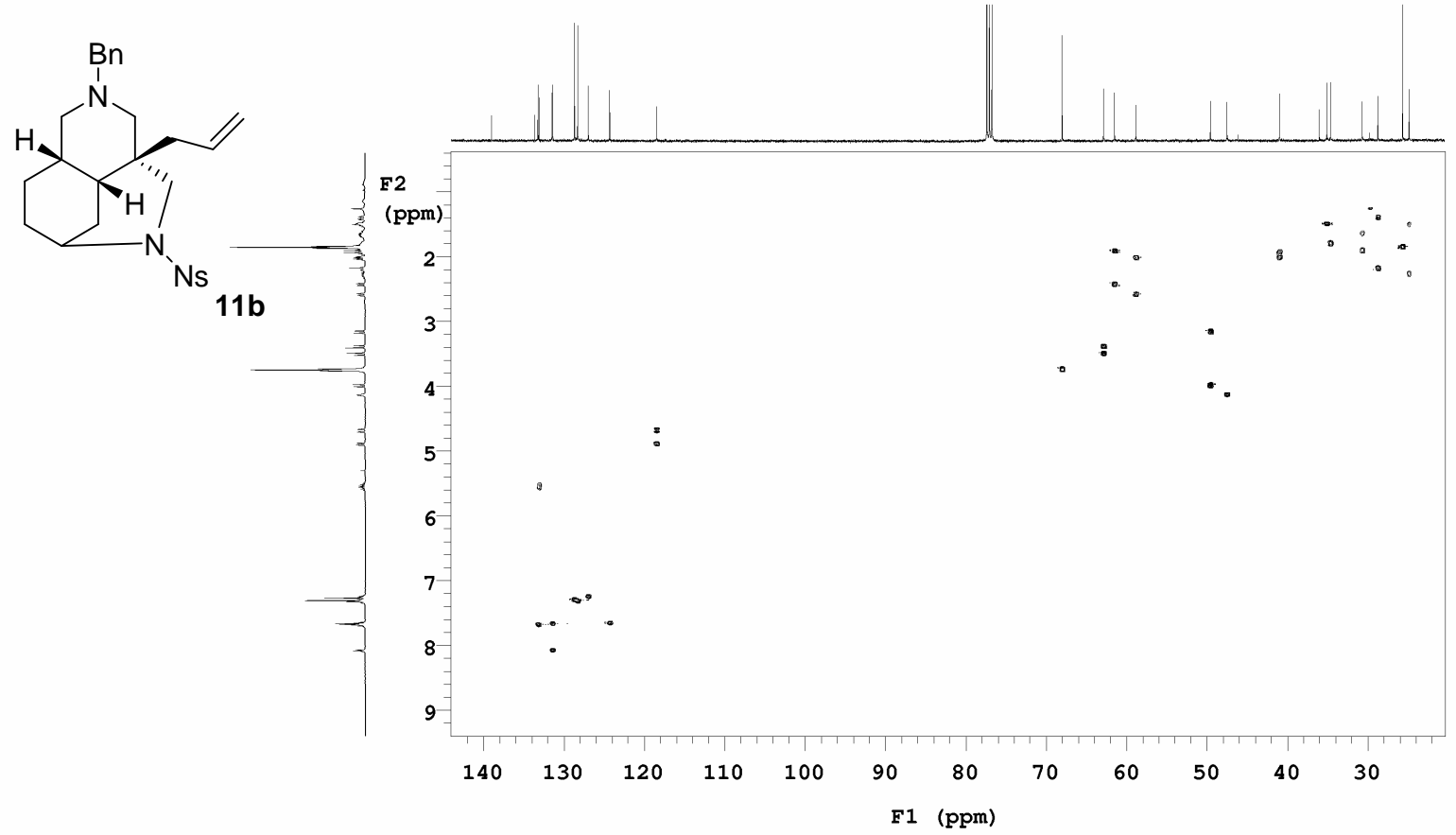

